\title{
Catechol-Based Biomimetic Functional Materials
}

\author{
Josep Sedó, Javier Saiz-Poseu, Felix Busqué, and Daniel Ruiz-Molina * \\ Dr. J. Sedó, Dr. D. Ruiz-Molina \\ Centro de Investigación en Nanociencia y Nanotecnología (CIN-CSIC) \\ Edifi cio CM7, Campus UAB, 08193 \\ Cerdanyola del Vallès, Barcelona, Spain \\ E-mail:druiz@cin2.es \\ Dr. J. Saiz-Poseu \\ Fundació Privada Ascamm \\ Parc Tecnològic del Vallès \\ Av. Universitat Autònoma \\ 23 - 08290 Cerdanyola del Vallès, Barcelona, Spain \\ Dr. F. Busqué \\ Departament de Química \\ Universitat Autònoma de Barcelona \\ Campus UAB- 08193, Cerdanyola del Vallès, Barcelona, Spain
}

\begin{abstract}
.
Catechols are found in nature taking part in a remarkably broad scope of biochemical processes and functions. Though not exclusively, such versatility may be traced back to several properties uniquely found together in the o -dihydroxyaryl chemical function; namely, its ability to establish reversible equilibria at moderate redox potentials and $\mathrm{pHs}$ and to irreversibly cross-link through complex oxidation mechanisms; its excellent chelating properties, greatly exemplifi ed by, but by no means exclusive, to the binding of $\mathrm{Fe} 3+$; and the diverse modes of interaction of the vicinal hydroxyl groups with all kinds of surfaces of remarkably different chemical and physical nature. Thanks to this diversity, catechols can be found either as simple molecular systems, forming part of supramolacular structures, coordinated to different metal ions or as macromolecules mostly arising from polymerization mechanisms through covalent bonds. Such versatility has allowed catechols to participate in several natural processes and functions that range from the adhesive properties of marine organisms to the storage of some transition metal ions. As a result of such an astonishing range of functionalities, catechol-based systems have in recent years been subject to intense research, aimed at mimicking these natural systems in order to develop new functional materials and coatings. A comprehensive review of these studies is discussed in this paper.
\end{abstract}




\section{Introduction.}

Catechols are benzene derivatives with two neighboring (orto-) hydroxyl groups, ubiquitously spread in nature. For example, catecholamine neurotransmitters, ${ }^{[1]}$ such as adrenaline and noradrenaline, fulfill essential and well-defined biochemical roles. But catechol derivatives can also be found as active species in a variety of environments, displaying a remarkable degree of chemical and physico-chemical versatility that has already inspired, and is still leading to, an ever-growing number of applications as functional materials. In this regard, this review will focus on past and recent studies involving the design and biomimetic use of synthetic catecholic molecules in biomedics, analytical (bio)chemistry, nanotechnology and materials science, showcasing the unique potential of this chemical functionality to afford multidisciplinary approaches to scientific research, while offering at the same time promising candidate structures for technologically relevant applications.

With regard to function, catechols may be broadly classified as follows:

- $\quad$ SWITCH, where catechol moieties function as responsive units to external stimuli

- SCAFFOLD, where catechol blocks build 2D or 3D structures, either by polymerization or self-assembly, which are functional on their own

- HOOK, in which catechol moieties make up complex systems in which they serve as individual binding sites to isolated molecules

- ANCHOR, where, either as part of complex systems or by themselves, catechol moieties act as passive links to surfaces and/or covalent supports to other (functional) moieties

Accordingly, this review has been structured in eight different sections, where the prominent functional role of the catechol moiety is first exemplified by natural counterparts. In Sections 1 to 4 , we will discuss the use of catechol derivatives to anchor molecules and polymers to an astonishing variety of surfaces (both nano/micro- and macroscopic), which currently constitute perhaps their fastest growing field of application. Section 5 will present studies where catechols are shown to polymerize in oxidizing conditions to build up complex, polymeric scaffolds with excellent properties as surface coatings and incipient potential as functional materials. The ability of catechol groups to coordinate effectively anionic and cationic species, but especially transition metals such as $\mathrm{Fe}^{\mathrm{III}}$, in therapeutic, analytical and environmental applications will be reviewed in Section 6. Finally, Sections $7 \& 8$ will deal with examples of the reversible redox activity of catechols, which together with their strong ability to couple to other molecules and metals, and their use as stimulus-controlled switches for device applications. 


\section{Adhesives.}

Mussel-adhesive proteins have been the subject of intensive scientific research over the past decades, ${ }^{[2,3,4,5]}$ particularly because of the remarkable ability of several marine invertebrates to strongly adhere to virtually all surfaces, -even low-fouling materials, such as paraffin and Teflon $^{\Theta_{-}},{ }^{[4,6]}$ and more distinctively, to do so in wet conditions that are typically conducive to adhesion failure. ${ }^{[4]}$ In order to pin down the source of this robust adhesion, detailed studies on the blue mussel (Mytilus edulis) and other sessile species ${ }^{[7,8]}$ have been carried out in the past years. To hold fast to surfaces, mussels secrete a transparent, gel-like proteinaceous substance that hardens with time into strong adhesive threads, with concomitant darkening. ${ }^{[9,10]}$ All five mussel foot proteins (Mefp) unique to the adhesive plaque, albeit diverse in structure, present relevant common features, such as recurring sequence motifs, ${ }^{[11]}$ but more importantly, varying amounts of the non-essential catecholic aminoacid DOPA (3,4dihydroxyphenylalanine), ranging from 2 to ca. $30 \%{ }^{[2,} 6^{10]}$ Either free, functionalized in simple variant forms, or as part of polypeptidic backbones, DOPA is widely distributed in most phyla of the animal kingdom, where it plays an astounding variety of biochemical roles. ${ }^{[7]}$ With regard to adhesion in Mefps, DOPA was identified by Waite and co-workers as unique in that it may serve two essential purposes: ${ }^{[12]}$ on the one hand, as a catecholic anchor, it is able by itself to chemisorb to surfaces by using diverse mechanisms, such as metal bidentate coordination ${ }^{[13,14,15,16]}$ and hydrogen bonding, ${ }^{[3,4} 4^{11]}$ on the other hand, it oxidizes easily -both by chemical and enzymatic methods- to the corresponding DOPA-quinone, which is then able to act as cross-linking unit, either by reacting with nucleophilic groups in the polypeptidic matrix by means of Michael-type additions, or via direct free-radical aryl-aryl couplings. $\left[4,6^{11,17,18]}\right.$ Other physico-chemical mechanisms, such as $\pi-\pi$ stacking, ${ }^{[19]}$ conformational rearrangements arising from tautomerization mechanisms on side chains following catechol oxidation, ${ }^{[20]}$ and the presence of other aminoacid residues in the polypeptidic backbone as coadjuvants of adhesion, ${ }^{[8,21,22]}$ have also been cited as contributors to overall cohesion and adhesion properties shown by Mefps. In an interesting twist of the versatility of catechol physico-chemistry, recent evidence suggested that bacteria may use catecholic siderophores -otherwise unrelated to DOPA units in Mefps- to attach themselves to surfaces in the initial stages of formation of biofilms. ${ }^{[23,24]}$

Early attempts at obtaining a wet adhesive biomimic of Mefps were aimed at synthesizing oligopeptidic analogues based on repetitive aminoacid sequences, and other adhesive proteins. ${ }^{[} 6,9^{25,26]}$ More recently, a simplified approach based first on random co-polymers with catecholic moieties ${ }^{[27,28]}$ and, alternatively, on carefully designed polymeric scaffolds, ${ }^{[29]}$ together with a careful study of the influence of key parameters -DOPA content, nature of adherend and oxidizing conditions- on the performance of the prospective adhesives, ${ }^{[27,30]}$ signified a conceptual leap that provided a solid basis for most recent investigations. For instance, it was found that whereas adhesion increases roughly proportionally with increasing DOPA content, ${ }^{[19,27]} O$-quinones exhibit much lower adhesion to metal surfaces than parent catechols, ${ }^{[18,19,27,30,31]}$ and virtually no adhesion to other surfaces, such as mica. ${ }^{[19]}$ Therefore, the successful design of adhesives based on macromolecular materials with pendant DOPA, -or by extension, catecholic- units would need to strike a balance between interfacial adhesion and bulk cohesiveness, by finding the degree of overall oxidation that affords an optimal mixture of catecholic and $o$-quinoid moieties. ${ }^{[6,27]}$ Even when alternative cross-linking 
mechanisms have been used, such as Fe ${ }^{\text {III'-mediated coordination }}{ }^{[32,33]}$ or photo-curing, ${ }^{[34]}$ optimal adhesion still called for a careful adjustment of the extent of cross-linking. ${ }^{[32,35]}$ By contrast, in cases where reactive functional groups in the adherend surface material were likely to link covalently to the oxidized moieties, such as pig skin ${ }^{[31]}$ and an aminefunctionalized Si substrate, ${ }^{[13]}$ an increase in the degree of oxidation was shown to enhance adhesion to the surface, with rupture values in tensile tests consistent with the breakage of covalent bonds formed in the cross-linking stage. ${ }^{[13]}$

This ability of catechol derivatives to interact with surfaces has been exploited by many scientists worldwide to prepare new synthetic functional adhesives and coatings (vide infra). However, understanding the basic behaviour and assembly of catechols on surfaces still remains a challenge. To gain more insight into this issue, Ruiz-Molina et al. have reported new basic studies studying the self-assembly and interaction of catechols with surfaces by combined theoretical calculations and scanning tunneling microscopy (STM), which allows the direct observation of the molecular self-assembly processes on surfaces with molecular resolution. ${ }^{[36]}$ The results indicates that the mechanism for the strong adhesion of catechols on surfaces is of energetic (interactions on the surface) but mainly of thermodynamic (solvent effects) origin, opening new insights into the behaviour of catechol molecules on surfaces as well on 2-D molecular suprastructures. Moreover, the thermodynamic control over the differential adsorption of an alkylcatechol and the nonanoic solvent molecules has been used to induce a new temperature-induced switchable interconvesion on surfaces with two different phases differing in their crystal packing that coexist upon increasing or decreasing the temperature. ${ }^{[37]}$

Alongside excellent specific monographs recently dedicated to this fast-growing subject, ${ }^{[10,38]}$ a survey of representative studies follows, covering recent advances in this research field, and representing a wide variety of potential applications with technological relevance.

\subsection{General purpose adhesives.}

Many studies describing more or less simplified synthetic catechol-based polymeric structures with recognizable biomimetic features have been published in the past decade. Payne and coworkers described the biomimetic modification of chitosan with dopamine under enzymatic conditions (tyrosinase). Instead of a protein, the authors chose a polysaccharide with reactive amino groups as suitable cross-linking matrix via Michael's-type adducts of the o-quinone moieties. Although relatively long reaction times were needed, the in situ reaction yielded chitosan-based water-resistant adhesives with shear strengths up to $400 \mathrm{kPa} .{ }^{[39]}$

Wilker et al. prepared a range of copolymers of styrene and 3,4-dihydroxystyrene, thus replacing the protein backbone with a saturated hydrocarbon chain, and retaining the catechol unit as the only functional moiety reminiscent of mussel adhesives. Using this very simplified approach, these synthetic polymers were oxidized in controlled conditions using a variety of agents, among which, those effective to cross-link the polymer ( $\mathrm{Fe}{ }^{\prime \prime \prime}$, periodate, permanganate and dichromate), showing the formation of adhesive layers. Despite their relatively low molecular weights, up to $1.2 \mathrm{MPa}$ adhesion shear forces were measured for the copolymer with an optimized composition. ${ }^{[28]}$ Very recently, the same authors reported the synthesis of a terpolymer of styrene, dihydroxystyrene and $p$-vinyltolyltriethylammonium chloride aimed at 
investigating the role of electrostatic charges in the bulk properties of mussel-mimetic adhesives, which in Mfp's may be played by lysine moieties. Adhesion values were found to be lower than those of commercial strong adhesives in dry conditions, but higher in wet conditions. Particularly, the introduction of ca. $7 \%$ of an electrically charged ter-monomer yielded a polymer with the highest degree of adhesion -2.8 $\mathrm{MPa}$ in dry, $400 \mathrm{kPa}$ in wet conditions on aluminum substrates-, which then decreased with increasing content of this monomer, probably due to electrostatic repulsion. It was concluded that electrostatic charge may provide additional surface interaction to increase the adhesion of mussel-foot proteins to rocky substrates. ${ }^{[22]}$ The same authors have recently investigated the optimization of the 3,4dihydroxystyrene/styrene ratio in the copolymer, and reported the strongest polymeric mussel protein mimic reported to date, with adhesion strengths on Al comparable to those of cyanacrylate glue (7 MPa). ${ }^{40}$

The Messersmith group prepared a novel synthetic composite inspired in the composition of nacre, based on a DOPA-Lys-PEG polymeric cement for the layer-by-layer deposition of ca. 300 nanometer-sized sheets of $\mathrm{Na}^{+}$-Montmorillonite clay. Results showed that even small amounts of the cement were enough to impart good mechanical properties to the composite. Furthermore, it was shown that the addition of Fe"' enhanced the mechanical properties of the material because of DOPA-mediated coordinative cross-linking. The Young modulus of the best composite approached $7 \mathrm{GPa}$, comparable to that achieved with single and multi-walled carbon nanotubes, although still lower than that of natural nacre (ca. $250 \mathrm{GPa}$ ). ${ }^{[41]}$ The same research group reported the use of a brush co-polymer to increase the interfacial shear stress between a metal wire and a polymer matrix. Small wires of NiTi and Ti-6Al-4V were coated with brushes of polymethylmethacrylate, anchored to the surface by ATRP polymerization using a brominated dopamine derivative. Coated wires were further cast on a PMMA block and subjected to a pull test to determine their adhesion to the matrix. Results showed that the use of the dopamine-based brush coating more than doubled the macroscopic affinity of the metal wires for the matrix, compared with uncoated wires. ${ }^{[42]}$ As part of their research on biocompatible adhesives, the adhesive properties of catecholic moieties were further investigated by synthesizing a block methacrylic acid /methylmethacrylate copolymer with grafted DOPA units. This polymer was then used to fabricate an adhesive elastic membrane, with which pressure adhesion tests on $\mathrm{TiO}_{2}$ and pig skin surfaces were carried out. After repeated contact/removal experiments, adhesion was shown to decrease, likely due to loss of adhesive material on the membrane that ended up more strongly bound to the adherend surfaces. Interestingly, while oxidation dramatically decreased adhesion to $\mathrm{TiO}_{2}$, it improved binding to pig skin, probably due to the formation of covalent bonds by chemical reaction between oxidized DOPA units and the tissue. ${ }^{[31]}$

The careful design of a mussel-foot-protein-mimetic polymer with grafted dopamine pendant groups enabled the design by the Messersmith group of dual biomimetic adhesive surface that combined the properties of both dry gecko- and wet mussel-foot adhesion. The peculiar features of gecko feet were modeled by imprinting roughness into an adhesive polydimethylsiloxane (PDMS) surface in the form of submicrometer pillars. This patterned, gecko-like adhesive surface was then coated with a 20-nm layer of a mussel-mimetic dopamine methacrylate/methoxyethyl acrylate copolymer. The adhesion forces of the coated patterned surface to silicon nitride, titanium and gold were measured by AFM in dry (air) and wet (underwater) conditions, and compared to those of an uncoated, patterned reference surface. The authors showed that, while the mussel-mimetic coating increases dry adhesion 
with regard to the uncoated reference, the most dramatic increase takes place in wet conditions, where dopamine-mediated surfaces on $\mathrm{Si}_{3} \mathrm{~N}_{4}$ increased adhesion by 15 -fold. Adhesion was also proved to be reversible, both in dry and wet conditions, over at least 1000 cycles without significant performance loss. ${ }^{[43]}$ In contrasting study, Washburn, Sitti and coworkers prepared gecko-inspired elastomeric microfiber arrays with mushroom-shaped tips, which were coated with a film of dopamine methacrylate/methoxyethyl acrylate co-polymer. It was shown from macroscopic -i.e., no AFM-scaled- measurements that the gecko-like coated surfaces adhered reversibly to glass and that gecko-like fiber patterns, coupled with the adhesive film, presented a cooperative effect that enhanced wet adhesion. In dry conditions, though, the expected contribution of the dopamine-based film on the adhesive properties of the gecko-like surface was found negligible ${ }^{[44]}$ The same research group later showed that the incorporation of a very small amount of a cross-linking third monomer -ethylene glycol dimethacrylate (EGDMA)- in the aforementioned co-polymer scaffold was sufficient to afford a viscoelastic material with significantly enhanced and reversible adhesion in wet conditions, ${ }^{[35]}$ that also improved the latter in coated gecko-like surfaces. ${ }^{[45]}$

Polymers with catecholic pendant groups have also been tested as adhesive re-inforcers. T.G. Park, Lee, and co-workers successfully improved the mechanical properties of carbon nanotube fibers (CNF) by infiltration of a star-branched polyethylenimine-catechol ${ }^{[46]}$ in the CNFs in the course of a post spinning treatment, which involved final densification of the coated CNFs by evaporation of the solvent and curing at $120^{\circ} \mathrm{C}$. The tensile strength of the fibers was shown to increase by 2 -fold solely by infiltration, and 4-fold (up to $2.2 \mathrm{GPa}$ ) after curing. Furthermore, tensile strength of the treated fibers was shown to increase as well with the catechol end-group content of the polymer. Finally, the mechanical strength of the fibers increased almost $500 \%$ with regard to untreated fibers by further cross-linking treatment with $\mathrm{Fe}^{\text {III }} \cdot[47]$

\subsection{Adhesive hydrogels for biomedical applications.}

As a specialty materials, adhesive hydrogels are much needed in many biomedical applications, such as stitch-less surgery, for which solubility in body fluids, sufficient wet adhesion, a degree of elasticity similar to that of soft tissues, and non-toxicity/biocompatibility are essential requirements for a satisfactory performance. ${ }^{[34,48]}$ Furthermore, for medical applications hydrogels should undergo a fast sol-gel transition once in contact with the tissue, in order to avoid surgical delay. Because of the exceptional wet adhesion of mussel adhesives and crosslinking abilities of catechol moieties, biomimetic hydrogels based on these have been researched in the past years and emerged as promising candidates to fill this challenging technological niche. ${ }^{[10]}$

In one of the first simplified designs of synthetic catechol-based wet adhesives, Deming et al. prepared random copolymers of DOPA and lysine, which were identified as the key components in the adhesive properties of marine mussel proteins. In this work, primacy was given to the straightforward incorporation of these aminoacids into a polymeric backbone, rather than on achieving specific, peptide-like sequences. Prospective polymers were applied from aqueous solutions to aluminum and steel surfaces, and cured in aerobic and anaerobic $\left(\mathrm{H}_{2} \mathrm{O}_{2}\right.$, tyrosinase) conditions. Once cross-linked, DOPA-Lys co-polymers showed strong adhesion to aluminum and steel surfaces, which was found proportional to the DOPA content 
and dependent on the choice of oxidation conditions and oxidizing agent. An important conclusion of this study was the observation that the oxidative mechanism involved in the cross-linking increased the cohesion of the adhesive, but at the same time oxidized catechol residues to the corresponding $o$-quinones, thus reducing the overall adhesion to surfaces. ${ }^{[27]}$

Following another simplified approach, Messersmith and co-workers designed different linear and star-branched frameworks with DOPA end-caps as adhesive/cross-linkable moieties, and biocompatible PEG for the hydrogel-forming central scaffold. Four different DOPA-capped PEG-polymers were synthesized, paying attention to the cross-linking mechanism resulting from each of the different oxidizing agents tested, both chemical- and enzyme-based. Most polymers were shown to form gels, and all were satisfactorily cured, with cross-linked structures made up of three to more than six units of the original framework. Gelation time was found to depend on the oxidation conditions and the architecture of the PEG chains. ${ }^{29]}$ The same group reported on a photopolymerizable DOPA-PEG-methacrylamide monomer intended to cure under UV irradiation. Without the need for oxidative cross-linking, this hydrogel was thus specifically designed to be less prone to lose adhesion by concomitant oxidation of the catecholic moieties. These photocured hydrogels showed elastic moduli considered suitable for biomedical applications. ${ }^{[34]}$ Using a similar strategy with a branched, DOPA-terminated, PEG-polylactic acid-methacrylate block polymer, a photocured, biodegradable hydrogel could be prepared with an elasticity similar to that of soft tissues, and good adhesion to titanium surfaces in wet conditions. As in previous works, adhesion to metal surfaces was shown to decrease in the presence of oxidizing agents. ${ }^{[30]}$

In more recent studies, Messersmith and co-workers have focused on the demonstration of the in vivo performance of catechol-PEG adhesive hydrogels. For example, a star-branched 4arm PEG hydrogel polymer terminated with dihydroxyhydrocinnamyl units, cross-linkable in ca. 30s upon addition of $\mathrm{NaIO}_{4}$ was tested on diabetic mice to seal islets transplanted to their extrahepatic tissue. Mice were shown to recover a normal glycemic response with minimal inflammatory response and unchanged contact with the supporting tissue for up to one year. ${ }^{[49]}$ The same hydrogel adhesive was shown to successfully seal punctures in model elastomeric membranes with an elastic response similar to that of typical fetal membranes. ${ }^{[50]}$ For a series of biodegradable adhesive polymers, a similar, star-branched design based on catecholic ends -dopamine, dihydroxycinnamic acid-, polyethylene glycol (PEG), and polycaprolactone $(\mathrm{PCL})$, was utilized. These polymers could be cast into films, and their mechanical properties, extent of swelling, and degradation rate were tailored through the composition of the polymers as well as blending with additives. When coated onto a biologic mesh used for hernia repair, these adhesive composites demonstrated wet-resistant adhesion properties, with strengths significantly higher than that of fibrin glue, ${ }^{[51]}$ and ca. $60 \%$ of that of a cyanacrylate-based adhesive. When a coating of this bioadhesive was applied to sutured porcine Acchiles tendons, the stiffness and failure load of the suture increased by roughly $30 \% \cdot{ }^{[52]}$

Stewart and co-workers have recently reported on adhesive hydrogels formed by complex coacervation. In one example, the authors showed that a mixture of a modified gelatin and a negatively charged copolymer of dopamine metacrylate and ethylphosphate metacrylate, gelates at basic pHs. The cohesion of the adhesive gel was enhanced by the presence of Ca" and $\mathrm{Mg}^{\prime \prime}$ cations, which were used to adjust the coacervation temperature to biological conditions. Cohesive strength was further enhanced by oxidative cross-linking with $\mathrm{NaIO}_{4}$. The 
authors indicated that this coacervate would biodegrade, which would make it particularly suitable for biomedical applications. ${ }^{[53]}$ In another study, an adhesive formed by complex coacervation of a copolymer of methacrylamide and an alquilammonium acrylamide (positively charged), and a terpolymer of dopamine metacrylate, methacrylamide and ethylphosphate methacrylate, was shown to gelate at $\mathrm{ph}>4$. The gel showed an adhesion force to hydroxyapatite up to ca. $40 \%$ that of cyanacrylate glues. ${ }^{[54]}$

T.G. Park and co-workers prepared an injectable hydrogel by cross-linking catechol-grafted hyaluronic acid and a thiol-capped Pluronic ${ }^{\circledR}$ polymer. Cross-linking was assumed to take place via Michael addition of the thiol groups to oxidized catechol (i.e. o-quinone) moieties. The hydrogels showed longer-lasting stability and improved adhesion to biological tissues. ${ }^{[55]}$ In a follow-up study, a hydrogel was synthesized by cross-linking catechol-derivatized chitosan and a thiol end-capped Pluronic ${ }^{\circledR}$ F127 - a block copolymer of ethylene oxide and propylene oxide-. The thiolated polymer was intended for fine-tuning of the gelation speeds to adequate values for good tissue-repair at biological temperatures. The resultant hydrogels showed excellent in vivo performance, with the chitosan scaffold conferring haemostatic properties, and the catechol pendant groups, improved solubility and strong adhesion to wet mucoses and tissues. ${ }^{[56]}$ Following a similar modular approach in which dopamine moieties provide anchoring points for functional polimeric coatings, Ishihara, Huang et al. recently fabricated a hemocompatible, polymetacrylic acid-dopamine metacrylate-phosphorylcholine metacrylate terpolimer that resists platelet adhesion, while robustly adhering to Ti surfaces intended for cardiovascular implants. ${ }^{57}$

\subsection{Metal cross-linked adhesives.}

The outer cuticle of byssal threads of marine mussels is made up mainly of mussel foot protein Mfp-1, a peptide that contains ca. 10-15\% of catecholic aminoacid 3,4-dihydroxyphenyl-Lalanine (DOPA) ${ }^{[58]}$ As extracted, mfp-1 is known to carry a certain amount of Fe', a sizeable fraction of which remains tightly bound to the protein even after treatment with strong chelators (EDTA) and acidic conditions. ${ }^{[59]}$ Waite and co-workers showed that both mfp-1 and several DOPA-containing oligopeptide fragments of this protein are able to coordinate Fe $\mathrm{III}^{\text {II }}$ forming bis-catecholato complexes at physiological $\mathrm{pH}$, and tris-catecholato complexes in slightly basic conditions. ${ }^{[60]}$ The high stability of these coordination complexes, reminiscent of those of siderophores, would explain how mussels are able to keep and enrich their byssus with this metal. The same authors suggested that, with as many as 150 DOPA residues per peptide molecule, mfp-1 in coordination with equivalent amounts of Fe" would afford a feasible cross-linking strategy, notwithstanding other possible mechanisms. ${ }^{[58]}$ The Wilker group studied DOPA-containing Mfp-1 \& Mfp-2 protein extracts by ESR after treatment with controlled amounts of $\mathrm{Fe}\left(\mathrm{NO}_{3}\right)_{3}$ in both aerobic and anaerobic conditions. It was shown that upon coordination with a third unit of DOPA and in the presence of oxygen, an ESR signal corresponding to an organic radical shows up, at the same time that the $\mathrm{Fe}{ }^{\mathrm{II}}$ signal decreases, indicating that upon full metal coordination, $\mathrm{Fe}^{\text {III }}$ is reduced to low-spin Fe". Concomitantly, an electron is transferred to a DOPA unit, generating a reactive semiquinone which can further react to generate a further cross-linked, covalent structure. Thus, in aerobic conditions, both

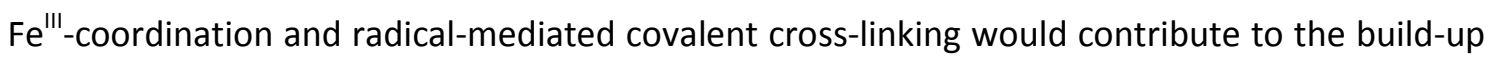
of mussel adhesive. ${ }^{[61,62]}$ Loizou et al. studied the effect of the addition of $\mathrm{Fe}^{\mathrm{III}}$ in the presence of oxygen on the morphologic and rheological properties of mussel adhesive protein extract. 
At the microscale, the initial porous hydrogel was seen to become more rigid and compact after the addition of the metal, which correlated with a more solid-like, elastic material. ${ }^{[63]}$ Further work on the mfp-1 protein showed that it is able to adhere to mica surfaces, but does not have the cohesive behavior necessary to glue two coated surfaces together. ${ }^{[64]}$ Nevertheless, the same surfaces could be bound together with the addition of a certain amount of Fe'". In this case, the metal must coordinate, to a certain extent, DOPA moieties belonging to $\mathrm{mfp}-1$ chains located on opposite surfaces, offering the necessary degree of cross-linking for adhesive cohesiveness. It was also shown that an excess of $\mathrm{Fe}^{\text {III }}$ was detrimental to cohesion, likely because the coordination number of the cation decreases when its concentration is raised. ${ }^{[65]}$ More recently, the Waite group showed that the inner structure of the byssal thread cuticle is a composite-like material made of an elastic protein matrix in which harder granules are dispersed. Using confocal Raman spectroscopy, it was found that the concentration of $\mathrm{Fe}^{\mathrm{III}}$ in the granules exceeded that of the matrix. Since differences in mechanical properties between phases correlate with the $\mathrm{Fe}^{\mathrm{III}}$ content in each phase, it was concluded that Fe" participates in the in vivo cross-linking of the protein. ${ }^{[6,67]}$ Moreover, because as opposed to covalent links, catechol-Fe"I bonds are strong, yet reversibly breakable, cross-linked proteins in byssal threads may likely self-heal in their natural environment by means of DOPA-Fe"II coordination. ${ }^{[68]}$ In another study, the application of computer aided design (CAD) to the patterning of various mussel adhesive proteins using piezoelectric inkjet technology was explored. $\mathrm{FeCl}_{3}$ solutions were applied as cross-linkers, and test performances were compared against those of commercial cyanacrylate glues. Cross-linking was found to be effective at stoichiometric Fe"IDOPA ratios, while adhesion decreased with $\mathrm{Fe}^{\text {IIII }}$ in excess. Although synthetic adhesives were still found superior in terms of strength, this study showed the potential of environmentally-friendly bioadhesives in microsutures. ${ }^{[69]}$

In addition to adhesive proteins from natural sources, a few synthetic mimics have been studied with regard to Fe'II cross-linking. A range of copolymers of styrene and 3,4dihydroxystyrene were prepared as simplified models of natural DOPA-containing peptides. After deprotection of the hydroxyl groups and controlled oxidation of the polymers with a variety of agents, Fe"II was found, among others, to cross-link the polymer forming adhesive layers. ${ }^{[28]}$ Fe"l'-mediated cross-linking was applied in a biomimetic fashion to the design of a synthetic self-healing hydrogel. A branched DOPA-modified PEG polymer was first mixed with $\mathrm{Fe}^{\mathrm{II}}$ in a 1:3 ratio at acidic $\mathrm{pH}$. After the initial development of a green color, typical of monocatecholato-Fe complexes, subsequent addition of controlled amounts of $\mathrm{NaOH}$ up to $\mathrm{pH}^{\sim 12}$ induced color changes, characteristic of the sequential formation of bis- and tris-DOPA complexes. Whereas the initial mixture was found to be essentially viscous at low $\mathrm{pH}$, the Fe $\mathrm{III}^{\mathrm{II}}$ cross-linked material had a sticky gel consistency and elastic behavior, with rheological properties close to those of a reference polymer alternatively cross-linked in a fully covalent fashion with $\mathrm{NaIO}_{4}$. More importantly, $\mathrm{Fe}^{\prime \prime \prime}$-cross-linked gels were self-healing while periodatecured gels were not, which was attributed to the reversible recovery of the Fe"-DOPA coordination network after shear-induced failure. ${ }^{[32]}$ Very recently, del Campo and co-workers reported on a biocompatible, Fe'II-crosslinkable hydrogel bearing 5-nitrodopamine moieties. In addition to the self-healing properties of this kind of metal-crosslinked polymers, the presence of the nitro group enabled the on-demand debonding of the adhesive upon light exposure. ${ }^{70}$

In addition to Fe', a self-healing effect via catechol-mediated cross-links has been achieved as well by taking advantage of the $\mathrm{pH}$-mediated, reversible nature of the boron-catechol bond. $\mathrm{A}$ branched catechol-derivatized PEG was reacted with 1,3-benzenediboronic acid at $\mathrm{pH}=9$ to 
yield a hydrogel cross-linked with boronate ester groups, which reverted to the uncross-linked, viscous liquid state after adjustment to $\mathrm{pH}=3$. The covalent nature of the cross-linked hydrogel state was found to be consistent with its rheological properties, showing stickiness and self-healing properties, sealing cuts seamlessly within 30 seconds. ${ }^{[71]}$ 


\section{Functionalizable platforms (Primers).}

Polydopamine has emerged in recent years as a virtually universal bio-inspired coating material and adhesive primer. Its use was pioneered by Messersmith and co-workers, ${ }^{[72]}$ who described an easy, straightforward coating method consisting of the aerobic auto-oxidation of dopamine in mildly basic aqueous buffered media, and in situ spontaneous deposition of the resulting polymer on a wide variety of substrates, ranging from inorganic (metals, metal oxides) to organic (polymers), including notoriously difficult materials, such as PTFE, with a remarkably wide-ranging effectiveness reminiscent of mussel adhesive proteins. ${ }^{[73]}$ More importantly, the authors showed that, in addition to its versatile coating abilities, polydopamine thin films allowed the immobilization of many organic species on top, opening up the possibility to use polydopamine-mediated chemistry as a generic way to coat substrates with functional organic adlayers. ${ }^{[72,74]}$ In this regard, it was assumed that successful binding of the top adlayer would involve Michael- and/or Schiff-base-type reactions between nucleophilic groups -such as amines and thiols- in the functional molecule and residual, electrophilic 0 quinoid moieties in the polydopamine film, resulting from the existence of at least a residual fraction of oxidized moieties in the polymer. ${ }^{[72]}$ More specifically, the Messersmith group demonstrated a generic strategy for the immobilization of biomolecules involving coating of the substrate by polydopamine, followed by reaction of nucleophiles -typically, amines or nucleophilic amine residues, which are common in complex biomolecules- with polydopamine film. In a competitive assay that employed lysine and hystidine, it was shown that it is possible to discriminate between nucleophiles by their respective rates of addition to the adhesive film. ${ }^{[75]}$ In addition to the ability to form covalent bonds with ad-layers, residual redox activity arising from coexisting catecholic and $o$-quinoid moieties in the polymeric backbone has been shown to play a significant additional role in the reactivity of polydopamine films. ${ }^{[76,77]}$ It is worth mentioning here that, notwithstanding its usefulness as adhesive primer, the structure of polydopamine is still subject of debate. In this regard, it has been recently related to that of eumelanin ${ }^{78}$ and, in contrast with the common understanding so far, it has been suggested to be essentially made up of monomeric units of 5,6-dihydroxyindoline and its quinoid derivative that self-aggregate by a combination of charge transfer, $\pi$-stacking, and hydrogen bonding interactions. $^{79}$

Although most of the work on polymeric adhesive primers by Messersmith's and other groups has favored polydopamine, norepinephrine has also been studied as an alternative tool for facile catechol-based surface modification. Because of their structural similarities, dopamine and norepinephrine are amenable to analogous oxidative polymerization methods. On the other hand, norepinephrine is complementary to dopamine in that it can easily activate by itself SI-ROMP polymerizations, thanks to the presence of a hydroxyl group in the side-chain. ${ }^{\left[{ }^{80]}\right.}$

In addition to the comprehensive list of materials included in Messersmith's original proof-ofconcept work, ${ }^{[72,74]}$ intensive research has been carried out in the past years regarding new coatable materials, -such as graphene oxide ${ }^{[76]}$ diamond-like carbon, ${ }^{[81]}$ carbon nanotubes, ${ }^{[82]}$ polylactic acid, ${ }^{[83]}$ polycaprolactone nanofibers, ${ }^{[84,85]}$ and yeast cells. ${ }^{[86]}$ On the other hand, many functional adlayers, like polymeric brushes, $\left.{ }^{[76,82,87}\right]$ aminoacids, ${ }^{[75]}$ avidin, ${ }^{[86]}$ heparin, ${ }^{[88,89,90]}$ hyaluronic acid, ${ }^{[72,91]}$ and functional living cells, ${ }^{[84,92,93,94,95]}$ have been successfully immobilized on polydopamine-coated substrates. With regard to its potential use in biomaterials, a very recent study on the in vivo toxicity of polydopamine showed that a one- 
step coating of poly-L-lactic acid with polydopamine greatly reduced the inflammatory response to such coated surfaces and the immunological responses of blood on coated quantum dots. ${ }^{[83]}$

\subsection{Polydopamine - General purpose applications.}

Kang, Fu and co-workers showed that dopamine may play a dual role, both as a reducing agent and as adhesive layer, in the preparation of functionalized graphene surfaces in mild conditions. Graphene oxide was coated polydopamine, which once in contact with the surface, reduced the GO to graphene. To this surface-modified graphene, PEG brushes were grafted using amino and thiol end-capped PEG, resulting in water-dispersible graphene. ${ }^{[76]}$ In a similar study, Lee et al. used polynorepinephrine to coat/reduce GO to graphene. Further treatment consisted of the polymerization of $\varepsilon$-caprolactone on top of the modified graphene by SI-ROP polymerization techniques. The residual redox activity of the coating was shown to be able to reduce $\mathrm{Ag}^{+}$to $\mathrm{Ag}$ nanoparticles, which ended up attached to the polymeric coating, providing the material with antibacterial properties. ${ }^{[77]} \mathrm{A}$ hybrid material was prepared by Zeng et al. by coating reduced graphene oxide with polydopamine and further incubating the modified surface in simulated body fluid. ${ }^{96}$

Wang, Yang and co-workers made use of both residual electrophilic and nucleophilic activity in polydopamine films to sandwich them in succession between a nucleophile bottom layer and an electrophile top layer. Surface $\mathrm{Si}-\mathrm{O}$ bonds on silicon were reacted with aminopropyltriexoxysilane. Once silanized, the surface was further coated with polydopamine. Finally, the double-coated substrate was treated with stearoyl chloride, which reacted presumably with pendant amino groups of dopamine. This three-tiered coating was shown to be very stable, which was attributed to the covalent links established between all layers. The micro- and macrotribological properties of the modified surface were studied, showing that the friction coefficient decreased significantly. ${ }^{[97]} \mathrm{A}$ similar strategy has been recently used by the same authors to assemble reduced graphene on silicon surfaces ${ }^{98}$. Very recently, Kim et al. prepared a stretchable, transparent and conductive polymer spray-deposition of silver nanowires on polydopamine-modified polydimethylsiloxane. ${ }^{99}$

Zhou and co-workers used carbon nanotubes $(\mathrm{CNTS})^{[82]}$ and $\mathrm{TiO}_{2}$ nanowires ${ }^{[87]}$ as platforms to immobilize brush polymers via dopamine chemistry. Firstly, CNTs were coated with a layer of polydopamine, which then reacted with a suitable thiolated initiator. Brushes of polydimethylaminoethyl methacrylate (PDMAEMA) were then grown on top of the polydopamine layer by SI-ATRP, and further modified by quaternization of the nitrogen atoms. These positively charged CNTs were placed in contact with $\mathrm{PdCl}_{4}^{-}$solutions and subject to electrochemical reduction, which induced the precipitation of Pd nanoparticles on the CNTs. Pd-loaded CNTs showed high electrocatalytic activity for the oxidation of methanol and hydrazine sulfate. In the case of $\mathrm{TiO}_{2}$ nanowires, a final thermal treatment was used to volatilize the polymeric brush and graft the Pd NPs to the surface of the $\mathrm{TiO}_{2}$ nanowires. When Au nanoparticles were added to the mix, Pd-Au alloy nanoparticles attached to the nanowires were obtained. ${ }^{[100]}$ The same research group investigated the electrodeposition of $\mathrm{Au}$ on a polydopamine-coated indium tin oxide surface. Polydopamine was shown to induce the crystallization of $\mathrm{Au}$ in a flower-like morphology, which turned out adequate for the use of surface-enhanced Raman scattering as a detection technique. Rhodamine $6 \mathrm{G}$ could thus be 
quantified at the gold surface in the pM regime. ${ }^{[101]}$ The in situ precipitation of $\mathrm{Au}$ in the presence of polydopamine thus afforded Au NPs embedded in the polymeric film. A very similar assemblage, based on AuNPs, polyaniline and polydopamine, was used by $\mathrm{Li}$ and coworkers to fabricate an electrochemical sensor for ascorbic acid down to the $\mu \mathrm{M}$ range. ${ }^{[102]}$ Very recently, Yin et al. devised a sensor for $\mathrm{H}_{2} \mathrm{O}_{2}$ by depositing polydopamine/Au NPs/horseradish peroxidase on a Au electrode. The authors claimed that the performance of the sensor was enhanced by the extra conductivity contributed by the Au NPs. ${ }^{[103]}$

Finally, polydopamine has been recently shown to be amenable to microcontact printing with polydimethylsiloxane (PDMS) stamps. Tsai et al. successfully imprinted polydopamine on a variety of substrates - glass, silicon, gold, polystyrene, and PEG - after deposition of the adlayer on PDMS. Polydopamine patterns were subsequently modified with a variety of functional layers, such as cells, bovine serum albumin, reduced $\mathrm{Ag}$ ions in the form of $\mathrm{Ag}$ nanoparticles, and Au nanoparticles. ${ }^{104} \mathrm{~A}$ very similar strategy for the immobilization of cells was reported by Zhang, Jiang and co-workers. ${ }^{105}$

\subsection{Polydopamine - Biomedical applications.}

A few recent works have started to tap into the enormous potential of polydopamine as biocompatible adhesive in the biomedical field, by showing that a wide variety of biologically relevant substances may be successfully immobilized on substrates of choice. For instance, Choi and co-workers used an indirect strategy to deposit yeast cells on a substrate, by coating them with a polydopamine layer, subsequently functionalized with an avidin adlayer. The modified polydopamine layers were shown to be stable, inhibit cell division, while at the same time conferring certain protection to the yeast cells against enzymatic aggression. On the other hand, a $\mathrm{TiO}_{2}$ surface was functionalized with biotin-capped-polyPEGMA. Thanks to the formation of avidin-biotin complexes, coated yeast cells could be effectively immobilized on the $\mathrm{TiO}_{2}$ surface. ${ }^{[86]}$ The direct immobilization of living cells has found incipient application in biosensing techniques. For instance, polydopamine layer on a Au/polypyrrole actuator was shown to enhance the adhesion of bacteria and their accumulation from physiological media. ${ }^{93]}$ In another report, Elimelech et al. showed that a single $E$. coli cell may be adhered to the tip of an AFM cantilever, once coated with polydopamine. Immobilized cells were shown to be alive by fluorescence techniques. ${ }^{[94]}$ Recently, Nam and co-workers coated a variety of substrates (Au, glass, Pt, ITO) intended for use as neural surface interfaces, with polydopamine, and later with poly-L-lysine (PLL). Neuron cultures were not viable on polydopamine-only films, but they grew normally on PLL ad-layers reacted with PDA coatings. Moreover, PLL-polydopamine-coated microelectrode arrays were readily functional, so that both spontaneous and stimulated neural activity could be recorded from cultured neuronal networks. ${ }^{[95]}$

H. Park et al. reported using a polydopamine layer to immobilize gelatin on a fibrous matrix of polylactide-co-e-caprolactone. The adhesion of gelatin -a potential carrier for therapeutic payloads- to the matrix was thus enhanced, without impairment of its mechanical properties. ${ }^{[85]}$ The adhesion of a lectin (concavalin A) on Au, In, Ir was demonstrated by Morris and co-workers by showing the selective binding of ribonuclease $B$ (RNase B) on the modified surfaces. ${ }^{[106]}$ Wang and co-workers immobilized bovine serum albumin (BSA) on diamond-like carbon surfaces via polydopamine, showing that modified surfaces present low cytotoxicity 
and good hemocompatibility. ${ }^{[81]}$ In a very similar study, Tao et al. modified a silicon surface with a biocompatible and corrosion-resistant BSA monolayer. ${ }^{[107]}$ When polydopamine was used to immobilize VEGF onto $\mathrm{Ti}$ alloy surfaces, a better attachment of human dermal microvascular endothelial cells (HDMECs) was observed, indicating that such modified Ti surfaces have potential for bone healing and regeneration applications. ${ }^{[108]}$ In a similar application, $\mathrm{TiO}_{2}$ nanotubes were grown on $\mathrm{Ti}$ surfaces and coated with morphogenetic protein 2 by using polydopamine as adhesive primer. Coated surfaces showed in vitro enhanced proliferation and differentiation of mesenchymal stem cells. ${ }^{[109]}$ Zhang and co-workers coated an $\mathrm{Au}$ electrode intended as a biosensor with polydopamine. First, anti-(sulfate reducing bacteria) antibodies were immobilized on top to provide specific binding sites for detection. Then, bovine serum albumin (BSA) was added to block non-specific binding sites. Detection was possible using electrochemical techniques down to 100 colony forming units (CFU)/mL. ${ }^{[10]}$ Polydopamine coatings were also chosen to immobilize $\mathrm{SiO}_{2}$ nanoparticles with antifungal activity by Ferreira et al. The NPs were first coated with dextran aldehyde, then surfacereacted with amphotericin $B$, and finally immobilized on glass surfaces with polydopamine. While the modified NPs themselves showed high antifungal activity -stronger than that of colloidal silver-, and reusability without loss of therapeutic activity, polydopamine coatings used for their immobilization were found neither hemolytic nor cytotoxic. ${ }^{[111]}$ Städler and coworkers prepared a complex sandwich of biolayers by sequentially coating glass surfaces with poly-L-lysine, liposomes with a fluorescent lipidic cargo, polydopamine and myoblasts immobilized on top. It was shown that cells could uptake fluorescent lipids through the polydopamine layer. This qualitative controlled release was observed by checking the rate of increase of myoblast fluorescence, which was lower with increasing thickness of the polydopamine coating. ${ }^{[112]}$ In another controlled release study, Shea and co-workers reported the coating of microspheres of polylactic-co-glycolic acid with several prospective matrices, such as polydopamine and many cationic polymers (ex. poly-L-lysine). Coated microspheres were subsequently loaded with plasmids and used to prepare layered scaffolds, intended for the in vivo controlled, sustained release and delivery of genetic material. Although the slowest initial release was observed with polydopamine, this coating later afforded the most sustained release of plasmids. ${ }^{[113]}$

T.G. Park and co-workers prepared complex multilayer coatings for modified metal stents to be used as controlled release supports. For the first layer -the one directly in contact with the metal surface- polydopamine was used in order to immobilize a second layer of heparin on top. To the heparin-coated metallic surface, the authors deposited a multilayer composed of anionic poly(lactic-co-glycolic acid) grafted hyaluronic acid (HA-g-PLGA) micelles encapsulating paclitaxel, and alternating heparin layers. In this design, heparin conferred antithrombogenicity and hemocompatibility, whereas paclitaxel was intended as antiproliferation drug to suppress hyper-proliferation of smooth muscle cells. By adjusting the amount of heparin and HA-g-PLGA micelles, the controlled release of paclitaxel could be finetuned and the proliferation of muscle cells, inhibited. ${ }^{[89]}$ In a parallel study, a hyaluronic acid layer was immobilized on a stainless steel stent by means of dopamine chemistry. On top of it, a series of layers of cationic plasmid DNA/polyethylenimine (PEI) polyplexes afforded controlled release of pDNA. ${ }^{[91]}$ C.B. Park et al. developed a simple procedure to micro-pattern adhesive polydopamine on non-adhesive substrates and showed that several types of living cells could be selectively immobilized on top of the patterned areas. It was suggested that this strategy may be used in the fabrication of biochips. ${ }^{[92]}$ The same research group spun 
polycaprolactone in 700-nm-diameter nanofibers and further coated them with polydopamine. The viability of the dopamine-coated material for use in vascular grafts was tested, showing that human umbilical vein endothelial cells (HUVECS) exhibited highly enhanced adhesion and viability, increased stress fiber formation, and positive expression of endothelial cell markers, comparing favorably to gelatin-coated $\mathrm{PCL}$ material. ${ }^{[84]}$ More recently, heparin has been successfully immobilized on polyurethane ${ }^{[88]}$ and polyethylene ${ }^{[90]}$ substrates in a robust way by pretreatment of these with dopamine. The modified substrates were shown to inhibit blood coagulation and platelet adhesion. Kohane and co-workers used polydopamine to improve the adhesion between body tissues and low-adhesion polymeric implants. Both polydopamine and polydopamine-PEG-RGD (adhesive peptide) composites were applied to PMMA surfaces -used in prosthetic cornea implants-, in order to test their adhesion to both collagen gels and soft tissue. Interestingly, polydopamine was shown to adhere tightly to collagen gels, while the peptide conjugate did not, but showed lower adhesion to tissue than the peptide conjugate. The conjugate coating significantly enhanced cellular proliferation of corneal epithelial cells and keratocytes without causing excessive secretion of pro-inflammatory cytokines. ${ }^{[114]}$

Dopamine has been used by Kang et al. as benchmark in the investigation of other bioadhesives. For instance, barnacle cement was tested alongside dopamine as anchoring material for the immobilization of a poly-2-hydroxyethylmethacrylate (PHEMA) adlayer on a stainless steel substrate, to which chitosan chains were further attached. Both surface modifications showed excellent and similar stability and antifouling properties. ${ }^{[115]}$ In another study, polydopamine and poly-p-hydroxystyrene (pPHS) were used as primers for several triazine-based polymers cross-linked with tioether linkages as suitable adhesives for fibermesh-assisted bone fracture repair. It was found that the use of (poly)dopamine as primer coating on the (fractured) wet bone enhanced the performance of the adhesive, but shear strength results were still lower than those of cyanoacrylate adhesives. Nevertheless, the cytotoxicity of the novel adhesive/primer combinations was very low, and cyanacrylate turned to perform better as stand-alone adhesive than embedded in a fiber-mesh, where both the dopamine- and pPHS-primed triazine-based composite performed much better. ${ }^{[116]}$ Lai et al. described the potential use of dopamine-coated magnetic NPs as adhesives for model contaminants in waste waters (such as bisphenol A) and bacteria (E. coli) to be later analyzed by capillary electrophoresis. ${ }^{[117]}$

Wang and co-workers have recently used polydopamine as substrate for molecular imprinting analysis, coupled to detection by means of a quartz crystal microbalance. Sensing crystals were coated with polydopamine in the presence of 1,3,5-pentanetricarboxilic acid, chosen as innocuous analog for highly toxic domoic acid. Once the surrogate analyte was removed from the imprinted coating, domoic acid could be successfully detected in solution down to $5 \mathrm{ppb}$, below maximum residual limits established in Western countries. ${ }^{[18]}$

Many reports have been published in the past years concerning the use of polydopamine as adhesive/encapsulation matrix for electrodes and sensors in general. Yin and co-workers deposited polydopamine to serve as an adhesive layer on a glassy carbon electrode and a matrix for halloysite nanotubes capable of incorporating $\mathrm{Ru}(\mathrm{bpy})_{3}{ }^{2+}$ via cation exchange. This enabled the fabrication of an electroluminescent hydrophillic electrode. ${ }^{[119]}$ A series of complex nanocomposites were prepared by Xie et al. using polydopamine, in order to entrap MWCNTs and coat a glassy carbon electrode, capable of sensing hydroquinone. More sophisticated variants of this nanocomposite coating were devised to detect glucose and 
prepare the biocathode of a membrane-less glucose $/ \mathrm{O}_{2}$ biofuel cell (BFC). ${ }^{[120]}$ The same authors reported a complex nanocomposite where polydopamine is used as a matrix for glucose oxidase, Pt NPs, Au NPs, and antibodies. This nanocomposite material was tested in model amperometric immunoassays to detect human immunoglobulin $\mathrm{G}(\mathrm{hlgG}) .{ }^{[121,122]}$

\subsection{Other catechol-based platforms.}

Messersmith and co-workers prepared a mussel-inspired synthetic polymer $[\mathrm{N}-(3,4-$ dihydroxyphenetyl)methacrylamide-co-aminoethylmetacrylamide] to design a universal method of substrate coating for the immobilization of DNA onto surfaces. The mechanism by which DNA ended up immobilized onto the polymeric layer was not ascertained, but the authors suggested, that as in the case of polydopamine, it might involve covalent bonds between quinone (oxidized) residues and amines \& thiols on the DNA probe, as well as noncovalent interactions between these, such as $\pi-\pi$ and hydrogen bonds. It was also hypothesized that both the catechol moieties on one monomer type and the free amine moieties on the other might contribute to versatile adhesion, in a manner reminiscent of Mfp5 , in which both DOPA (catechol-terminated) and lysine (amine-terminated) residues make up $50 \%$ of the total amino-acid content. ${ }^{[123]}$

T.G. Park and co-workers functionalized a polyethyleneimine cationic polymer with dopamine end-caps and dopamine units grafted to an anionic hyaluronic acid polymer. These oppositecharge polymers were then successfully deposited in a layer by layer approach on surfaces, such as PTFE, that usually present resistance to coating, and to LbL coating in particular, because of their antiadhesive properties. Other challenging surfaces that usually need priming prior to coating, such as PE, PET, and polycarbonate (PC), could be successfully modified as well. Finally, the latent redox activity of the dopamine moieties was used to anchor $\mathrm{Ag}$ nanoparticles, which provided the coated surface with antibacterial properties. ${ }^{[46]}$ Catecholcapped polyethyleneimine polymers were used very recently by Lee, Jang et al. to immobilize adeno-associated viruses (AAV) on substrates in micro-patterns. Cells deposited within modified areas were selectively infected by viruses, as opposed to outlying cells. With regard to spatial control of the viruses, patterns could be "drawn" with a micropipette, as well as imprinted with a PDMS stamp. ${ }^{124}$

Other than monomeric catecholic initiators for SI-ATRP, reviewed elsewhere in this paper, Zhou et al. reported on a polymeric macroinitiator for SI-ATRP, bearing dopamine groups for surface anchoring and reactive groups intended for growing functional polymeric brushes, like temperature-sensitive poly( $\mathrm{N}$-isopropyl acrylamide). According to the authors, polymeric initiators provide stronger interactions with surfaces and higher stability than small- molecule initiators studied so far. ${ }^{125}$

Using a biomimetic approach, Payne and co-workers focused their investigations on the enzyme-assisted cross-linking of chitosan with phenols, in which o-quinone electrophilic intermediates were likely formed by the oxidative reaction of tyrosinase, either with phenolic residues in proteins -as a versatile strategy to cross-link proteins and polysaccharides-, or with added phenols intended as cross-linking moieties. In the first case, it was possible to conjugate untagged and hystidine-tagged organophosphorous hydrolase, cytochrome $\mathrm{C}$ and an 
acetyltransferase to chitosan. ${ }^{[126]}$ When gelatin was used, the resulting material was found to have gel-like properties. ${ }^{[127]}$ Chitosan was also cross-linked with several phenols and polyphenols (including catechol and catechin) electrochemically, on top of a silicon wafer micropatterned with gold, acting as electrode. The micropatterned cross-linked films were flexible and mechanically stronger than the parent chitosan film. Micropatterning could be controlled over large surfaces with a resolution down to $20 \mu \mathrm{m} .{ }^{[28]}$ 


\section{Macroscopic surface functionalization.}

\subsection{Antifouling coatings.}

Messersmith and co-workers reported a water-soluble polymer made of three alternating units of DOPA and lysine attached to a 20-unit N-methoxyethylglycine antifouling peptoid. The anchoring (DOPA-Lys) ${ }_{3}$ end, a simplified mimic of the structure of mussel adhesive protein Mefp-5, was shown to provide strong adhesion of the polymer to titanium surfaces by means of a simple immersion procedure. The antifouling peptoid moiety conferred long-term resistance to the attachment of human serum proteins and cultured cell fibroblasts to the coated surface. ${ }^{[129]}$ Similar polymers, but using only one, two or three DOPA units as anchor moieties for PEG, also showed resistance to serum protein adsorption on $\mathrm{TiO}_{2}$ surfaces. Fouling resistance was found to correlate with the number of co-joined DOPA residues at the anchorend, with PEG-DOPA 3 affording the highest packing density. ${ }^{[130]}$ In a follow-up study, the performance of PEG-DOPA 3 on $\mathrm{TiO}_{2}$ with regard to fouling resistance against certain species of diatom and green algae was found at least equal to, or better than that of a commercial polydimethylsiloxane (PDMS)-based coatings. ${ }^{[131]}$ The same research group reported the first use of a catecholic initiator in a surface-initiated (SI) atom transfer radical polymerization (ATRP). The dopamine-based initiator was first anchored to a Ti surface, and subsequently reacted with OEG methacrylate, to yield brush-like, OEG-terminated antifouling coatings up to $100 \mathrm{~nm}$ thick. By using standard photo-mask lithography, Ti surfaces were further patterned into coated (antifouling) and uncoated (cell-adhesive) areas, opening up the possibility of using these surfaces for cell array design. ${ }^{[132]}$

In another biomimetic approach, Gademann and co-workers prepared a series of synthetic analogs of the catecholic chromophore moiety of siderophore anachelin, and conjugated them with PEG. An interesting feature of the catecholic anchor employed was the presence of a quaternized amino group, which made the anchor resistant to oxidation, as opposed to dopamine-based moieties. Furthermore, the positive charge on the anchor was suggested to increase its affinity to negatively-charged surfaces. Polymer coatings on glass and $\mathrm{TiO}_{2}$ surfaces were found resistant to cell and protein fouling, and could be made thicker than dopaminebased ones. ${ }^{[133]}$ Dopamine-based anchors amenable through simpler synthetic procedures were also prepared. Among these, an anchor featuring a quaternized amine afforded the polymer adlayer with highest packing density, with antifouling properties comparable to those of a similar polymer with two non-quaternized dopamine-based anchors. ${ }^{[134]}$ Further research on anachelin-anchored polymers involved functionalization of the PEG end with antibiotic vancomycin, which added strong antimicrobial activity to the coating. ${ }^{[135,136]}$

In addition to catechol-ended PEG polymers, diverse polymeric backbones with grafted catecholic moieties have been studied. Zürcher and co-workers grafted PEG and 3,4dihydroxyphenylacetic acid to a polylysine backbone. The polymer self-assembled spontaneously and electrostatically-driven on $\mathrm{TiO}_{2}$, showing excellent antifouling resistance. This was preserved even in strong electrolyte media, even though the rest of the polymer desorbed because of the electrostatic screening effect of the electrolytes. By comparison, a similar polymer to which only PEG was grafted, quickly desorbed in the same conditions, proving that the role of surface-independent adhesion of the catecholic moieties is essential. ${ }^{[137]}$ Because of the presence of multiple anchoring units distributed along the 
polymer backbone, the graft-to approach was employed successfully in widening the scope of surfaces and materials (metals, oxides, PTFE) to which PEG-modified polymers could be robustly attached, without impairment of the surface-modifying capabilities and the high resistance to fouling by protein and cell adhesion of PEG-ylated polymers. ${ }^{[138]}$

A third architectural strategy, involving the use of dendritic PEG polymers, was reported recently by Textor and co-workers. PEG dendrimers were covalently attached to anchors based on assemblages of one or two- or three-covalently bound catechol groups. When at least three catechol groups were present, the dendrons attached fast and irreversibly to $\mathrm{TiO}_{2}$ surfaces as monolayers with persistent antifouling properties. Despite presenting lower levels of hydration in aqueous media, dendritic PEGs conferred antifouling properties comparable to those of linear-PEG groups. Furthermore, they afforded denser packings at lower temperatures and electrolyte concentrations. ${ }^{[39]}$

Finally, a multifunctional coating was prepared in which the antifouling monomer was able to immobilize specific biomarkers at the same time. Polymer brushes with anchoring DOPA units at one end and carboxybetaine on the other side were grown on gold surfaces. In addition to exhibiting excellent anti-fouling properties, antibodies of an activated leukocyte cell adhesion molecule (ALCAM) were successfully immobilized on this coating, giving rise to detectable surface plasmon resonance signals when the surface was exposed to biological media containing this cancer biomarker (ALCAM). ${ }^{[140]}$

\subsection{Antibacterial coatings.}

Detrembleur and co-workers have recently reported on two different antibacterial coatings assembled on stainless steel surfaces using a layer-by-layer approach. Antimicrobial films were fabricated, consisting of alternating layers of a polycationic copolymer with pendant units of DOPA and a quaternary ammonium salt, and an anionic polystyrene sulfonate, either flatlayered ${ }^{[141]}$ or arranged in layers of micelles. ${ }^{142}$ DOPA units in the bottom layer ensured good adhesion to stainless steel surfaces. On top of it, the middle layers of the polycationic copolymer were placed in contact with an $\mathrm{Ag}^{+}$solution, which induced the partial precipitation of nanoparticulate $\mathrm{Ag}$, so that elemental $\mathrm{Ag}, \mathrm{Ag}^{+}$and precipitated $\mathrm{AgCl}$ ended up embedded in the film, providing antibacterial activity. Even after depletion of the $\mathrm{Ag}$, the polycationic layers provided residual antimicrobial activity, which could be reactivated by immersion of the coated surface in a $\mathrm{AgNO}_{3}$ solution. In a second study, the same polycationic copolymer was used as a bottom layer, while the middle section was replaced by layers of oxidized (fullyquinoid) DOPA-polymethacrylate and polyallylamine (PAA), alternatively laid on top of each other. It was found that the amine residues of PAA reacted with the quinone residues in the other polymer, thereby cross-linking the successive layers with each other. For the top five layers, oligopeptide nisin -known for its anti-Gram positive antibacterial properties and nontoxicity- substituted for PAA. Despite its complexity, the coating was assembled on stainless steel without oxidizing agents and in mild, aqueous conditions in only $1 \mathrm{~h}$, and showed strong antibacterial activity after $24 \mathrm{~h} .{ }^{[143]}$ Using a simple strategy, Rahimipour and co-workers recently coated a variety of substrates with polydopamine and further reacted the adlayer with low-toxicity agent - a modified ultrashort lipopeptide with antibacterial activity-. The coating was shown to be robust and effective against E. coli. ${ }^{144}$ 
In addition to anchoring and antibacterial moieties, the incorporation of a third functional unit in the polymer may afford the adjustment of critical physico-chemical parameters. In a recent report, a terpolymer of dopamine-metacrylate, a long-chain-alkyldimethylethyloxyammonium metacrylate (biocide) and methoxyethylmetacrylate (hydrophobic/hydrophilic fine-tuner) was prepared. This polymer was shown to be tunable to a wide variety of polarities, which should allow the optimization of antimicrobial activity. ${ }^{[145]}$

Using a simple approach, $\mathrm{Xu}$, Mao et al., rendered cotton fabric antimicrobial by coating with polydopamine and subsequent deposition $\mathrm{Ag}$ nanoparticles by in situ reduction of $\mathrm{Ag}^{+}$. The fabrics showed strong antibacterial activity after 30 washes. ${ }^{[146]}$ In an elaborate example of multifunctionality, $\mathrm{Xu}$ and co-workers attached dehydrocaffeic acid (DHDCA) to $\mathrm{TiO}_{2}$ nanoparticles, to which $\mathrm{Ag}$ nanoparticles had been previously grafted. $\mathrm{TiO}_{2}$ - $\mathrm{Ag}$ modified nanoconjugates were then treated with DMDHEU -an anti-wrinkle agent for cotton fabrics-. Cotton samples dipped in an aqueous suspension of this DMDHEU-DHDCA modified $\mathrm{TiO}_{2}-\mathrm{Ag}$ nanoconjugates, once dried and over-cured, achieved a high-grade durable-press rating, while showing as well antibacterial properties. ${ }^{[147]}$ Following a similar strategy on a different substrate, C.K. Lee et al. obtained an antibacterial nanocomposite of bacterial cellulose, polydopamine, magnetite NPs, and precipitated Ag NPs. ${ }^{[148]}$

Research on suitable coatings that selectively inhibit bacterial adhesion but at the same time promote the growth of beneficial host cells was reported by Neoh and co-workers. In an early study, dopamine was used as an anchor moiety for tripeptide- and collagen-based coatings. It was shown that immobilized collagen promoted the adhesion of both fibroblast and Staphyloccocus aureus, whereas the immobilized RGD tripeptide sequence promoted fibroblast adhesion without any apparent effect on E. coli or S. aureus adhesion. ${ }^{[149]}$ More recently, this group reported the functionalization of two antibacterial polysaccharides carboxymethylchitosan and hyaluronic acid-, using dopamine as anchoring points, and vascular endothelial growth factor (VEGF) as promoter of osteoblast formation. Test results indicated that osteoblast growth was favored by this multifunctional coating, which concomitantly inhibited the adhesion of Staphylococcus aureus. ${ }^{[150]}$

In their continuing investigation of antifouling DOPA-polycarboxybetaine coatings, Jiang and co-workers successfully added biosensing capabilities to gold ${ }^{[140]}$ and $\mathrm{SiO}_{2}^{[151]}$ surfaces. Antibodies of an activated leukocyte cell adhesion molecule (ALCAM) were immobilized on the polymeric coating, giving rise to detectable surface plasmon resonance signals when the modified surface was exposed to biological media containing this cancer biomarker (ALCAM), while at the same time showing excellent resistance to protein fouling in $100 \%$ human plasma and serum. This multifunctional approach was said to overcome non-specific protein uptake, which often severely limits the use of biosensors in biological media. ${ }^{[151]}$

\subsection{Anti-corrosion \& anti-wear coatings.}

An early report by Waite and co-workers presented model corrosion studies showing that mussel foot protein is a good corrosion inhibitor of stainless steel, as compared with bovine albumin, 3,4-dihydroxybenzoic acid and polylysine. Interestingly, a synthetic analog of the $\mathrm{mfp}$ not only did not inhibit corrosion, but enhanced it. It was suggested that the secondary structure of the natural mfp might be playing a significant role in the inhibition mechanism. ${ }^{[152]}$ 
In a more recent attempt to confer corrosion resistance to metal surfaces, Yang et al. functionalized aluminum surfaces with a mercaptopropyl-terminated silane, which was in turn reacted with dopamine. Finally, dopamine moieties were covalently linked to tetradecyl pendant groups. Whereas a bilayer of siloxane-dopamine already enhanced the corrosion resistance of the material by 2 orders or magnitude in saline solutions, once completed with a hydrophobic layer on top, this resistance was roughly three orders of magnitude higher than that of bare Al. Nevertheless, the anticorrosion resistance in test conditions decreased sharply after two days, likely due to the loss of some tetradecyl side chains and exposure of the hydrophilic dopamine coating. ${ }^{[153]}$ Using a different approach, Detrembleur et al. studied the LbL deposition on galvanized steel of clay and a policationic copolymer of dopamine and an alkyl quaternary ammonium salt. This assemblage provided an effective barrier against corrosion that avoided the use of toxic chromic treatments. ${ }^{154}$

In order to explore the usefulness of dopamine in anti-wear applications, Zhou and co-workers used it as an anchor on Si surfaces for an imidazolium-based ionic liquid. The authors showed that the wetting of the modified surface could be tailored by choice of an appropriate counterion. On the other hand, the tribological properties of a Si surface directly spin-coated with the ionic liquid, a second surface coated with a dopamine-ionic liquid SAM, and a third one in which the imidazolium-based ionic liquid was spin-coated on top of a dopamine-ionic liquid SAM were compared. It was shown that while both the bare SAM and the surface directly spincoated with the ionic liquid presented significant wear, the composite coating performed much better in terms of both wear resistance and load-carrying capacity. ${ }^{[155]}$ In a different study aimed at the development of novel water-based lubricants, the same authors reported the coating of PTFE powder with polydopamine. In addition to water dispersibility, the performance of a $0.8 \% \mathrm{w} / \mathrm{v}$ water suspension of the dopamine-modified PTFE powder as lubricant was compared with that of distilled water. For the suspension, the friction coefficient remained stable at ca. 0.1 after 30', whereas that of water increased dramatically within 5 $\min ^{[156]}$

\subsection{Hydrophobic/hydrophilic coatings.}

Polydopamine offers a simple, generic and straightforward way to radically modify the surface energy of materials, either directly by making them hydrophilic or, alternatively, as a mediator coating for hydrophobic ad-layers. Chen et al. made a copper surface water-repellent by modification with polydopamine, followed by reaction of the ad-layer with dodecanethiol. In addition to hydrophobicity, the modified surface was found to be corrosion-resistant in saline media, likely because it limited the infiltration of $\mathrm{Cl}^{-}$anions. ${ }^{[157]}$ Park, Choi and co-workers rendered porous polyethylene sheets hydrophilic with polydopamine, without affecting the degree of porosity of the substrate. This way, the material, intended for separators in Li-ion batteries, could be easily soaked with polar electrolyte fluids -e.g. propylene carbonate-, and used to prepare a model cell that showed improved electric performance, such as lower resistance and greater discharge current densities after repeated cycles. ${ }^{[158]}$ The amphiphilic character of polydopamine films, which may be positively charged by protonation of the indolic nitrogen atoms or negatively charged by deprotonation of unoxidized catecholic hydroxyl groups, was put to use in the fabrication of permselective electrodes. Au-coated Si wafers, additionally coated with polydopamine, were found to selectively block cations at $\mathrm{pH}<3$, and anions at $\mathrm{pH}>11$. The $\mathrm{pH}$-dependent permselectivity was shown to be fully 
reversible and was characterized by cyclic voltammetry using redox-active probes. ${ }^{[159]}$ Polydopamine afforded as well an interesting combination of adhesion and superhydrophobicity on the same material. Lee and co-workers micro-patterned a superhydrophobic polyfluorosiloxane-coated anodic aluminum oxide surface with capillary channels of adhesive, hydrophilic polydopamine. Water droplets were shown to stick to the patterned surface, which was able to hold them, even when it was flipped upside down. At the same time, the patterned substrate still kept a high water/surface contact angle, typical of superhydrophobic surfaces. This material was used to fabricate a water capturing device reminiscent of the water-collection technique of the desert beetle. ${ }^{[160]} \mathrm{A}$ similar approach that combined polydopamine-coated hydrophilic micro-troughs patterned on superhydrophobic anodized aluminium oxide (AAO), was used by the same authors to devise a purely gravitydriven microfluidics device for the channeling of aqueous droplets into chemical reactions in the microscale. The high channeling efficiency of this pump-free mixing device afforded remarkably high reaction throughputs. ${ }^{161}$

Hydrophobicity and superhydrophobicity on surfaces was also achieved by covalently linking perfluoroalkyl chains to catecholic anchors, or grafting fluorinated chains and catecholic anchors to polymer backbones, respectively. Spencer et al. coated $\mathrm{TiO}_{2}$ surfaces with SAMs of a perflouroalkylated derivative of 6-nitrodopamine. Surfaces showing water contact angles of up to 1050, plus time-dependent hydrophobic gradients could be achieved by controlling the exposure time of the surface to the coating solution. The use of the nitro derivative was judged essential, since its electron-withdrawing character ensured that catechol moieties wouldn't oxidize easily and, accordingly, true catechol SAMs -instead of randomly cross-linked polymerscould be obtained. ${ }^{[162]}$

Zhou and co-workers prepared catechol-anchored, grafted fluoropolymers using two different strategies. In one instance, a copolymer of DOPA-methacrylate and a perfluoroalkyl methacrylate was synthesized. This fluorinated polymer attached itself to a variety of surfaces ( $\mathrm{Ti}, \mathrm{Al}, \mathrm{Cu}, \mathrm{Si}$, steel, glass, and organic polymers), which exhibited low surface energies and high water contact angles (ca. 120) consistent with high hydrophobicity. When roughness was added to a surface of alumina in the form of nanowire forests, contact angles went up to 165ㅇ (superhydrophobicity). ${ }^{[163]}$ In another study, the authors chose a catechol derivative both as anchor and activator for surface-initiated polymerization by ring-opening metathesis (ROMP) of a norbornene spacer with a perfluoroalkyl pendant group. The coating was successfully carried out on a variety of surfaces, such as $\mathrm{TiO}_{2}$ nanowires, $\mathrm{Al}_{2} \mathrm{O}_{3}, \mathrm{Ag}$, $\mathrm{Zn}$, and many others. As in the previous study, surface-modified $\mathrm{Al}_{2} \mathrm{O}_{3}$ nanowires showed superhydrophobic behavior. ${ }^{[164]}$ The same research group reported on the use of polydopamine-coated mesoporous silica NPs as reservoirs for the controlled release of a hydrophobic molecule (octadecylamine). When substrates coated with these NPs were etched so as to remove the hydrophobic coating, underlying octadecylamine migrated to the surface and bound chemically to the polydopamine layer. Thus, the hydrophobic character of the surface was restored in $24 \mathrm{~h}$ in a self-healing fashion that could be cycle at least 25 times without apparent loss of surface properties. Moreover, thanks to the the rough microstructure of the surface, the coating afforded water repellency in the superhydrophobic range. ${ }^{165}$

A couple of studies have been recently reported concerning the modification of surface properties with polydopamine in practical applications. Yin and co-workers coated the inner surface of an open-tubular capillary column intended for capillary electro-chromatography, 
and found that the electro-osmotic flow could be better controlled, compared to an uncoated column, thanks to the residual, basic hydroxyl and acid amino groups in the polymer. In addition, the polydopamine coating afforded an improved resolution in the separation of four auxines ${ }^{[166]}$ Hydrophilicity was conferred to hydrophobic polysulfone membranes intended for reverse osmosis by means of a plain polydopamine coating, resulting in a significant improvement in osmotic flux performance. The authors remarked that this approach hydrophilic coating of hydrophobic, tough polymers- should be advantageous in this application as opposed to the use of intrinsically hydrophilic polymers, which would be prone to swelling and plasticizing upon aqueous wetting. ${ }^{[167]}$ 


\section{Micro-/Nanoscopic surface functionalization.}

Iron oxide nanoparticles (NPs) have been researched extensively in recent years because they provide many potentially useful properties, such as superparamagnetism and cellular uptake, which are essential to MRI imaging and other biomedical applications. For magnetic NPs to play a successful therapeutic role, they should be stable and homogeneously dispersed in biological fluids, not readily recognized and disposed of by the immune system, and be efficiently directed to, and internalized by specific targets. ${ }^{[168]}$ Since catechol derivatives are efficient chelators of ferric ions, they constitute a natural choice as robust surface anchors for magnetic NPs. Particularly, dopamine offers, in addition to a catechol moiety, a functional endgroup amenable to cost effective derivatization procedures. ${ }^{[169]}$

Although relevant non-biomedical applications have been explored, ${ }^{[170,171,172]}$ catecholmodified ferric NPs were intended in most cases for biomedical applications in which the magnetic properties of the NP core are themselves useful for MRI imaging, ${ }^{[173,174,175,176,177,178]}$ or else NPs act as carriers for therapeutic payloads, either to scavenge pollutants in body fluids, ${ }^{[179]}$ or to be selectively uptaken by diseased cells. ${ }^{[180,181,182,183,184]}$ Since dopamine may be functionalized in an easy, straightforward fashion, it has been the anchor moiety of choice for the majority of these studies, although DOPA, ${ }^{[174,181]}$ nitroDOPA, ${ }^{[173,184,185]}$ and dihydroxybenzylamine, ${ }^{[186]}$ among other catechol derivatives, have also been used to this effect. Conjugated to the catecholic anchor, PEG was chosen almost exclusively as biocompatible stabilizer of NPs in physiological media, in addition a few others, such as hyaluronic acid, ${ }^{[177]}$ oligopeptidic chains ${ }^{[171]}$ and poly(carboxybetaine methacrylate). ${ }^{[174]}$ In several cases, extra functional moieties, such as specific chelating agents, ${ }^{[170,178,179,186]}$ porphyrin, ${ }^{[180]}$ and therapeutic drug moieties, ${ }^{[182,183]}$ were covalently linked at the end of the PEG chain or directly conjugated to the anchor. In addition to the bibliography commented in this Section, a very recent review on the role catechol derivatives in iron oxide NP functionalization should provide the reader with additional details on this topic. ${ }^{187}$

\subsection{Colloid stabilization.}

In an early report, Sun and co-workers coated $\mathrm{Fe}_{3} \mathrm{O}_{4}$ nanoparticles with a PEG-triazinedopamine conjugate, which afforded a convenient synthetic route to link together PEG chains and dopamine anchors. The stability of nanoparticle dispersions in aqueous buffered media with high saline concentration was satisfactory. ${ }^{[169]}$ In a follow-up study, 9-nm NPs were functionalized with PEG-dopamine, but in this case standard EDC/NHS coupling chemistry was used instead in order to conjugate PEG and dopamine moieties. The functionalized NPs didn't aggregate after a $24 \mathrm{~h}$ period of incubation in PBS solutions and showed decreased uptake by macrophage cells, which suggested that they would escape the immune system in potential biomedical applications. ${ }^{[175]}$

Reinhult and co-workers coated $\mathrm{Fe}_{3} \mathrm{O}_{4}$ nanoparticles $(30 \mathrm{~nm})$ with nine different PEG-based dispersants and found that nitroDOPA and nitrodopamine anchors afforded the highest colloidal stability in physiological media even at high temperatures and dilutions. ${ }^{[173]}$ The same authors investigated the effect of the ring pendant groups on the bonding affinity of several catechol anchors. It was found that substituted nitrocatechols bind to Fe" sites, which transfer 
one electron that ends up delocalized in the nitroaryl moiety. These complexes were found to be stable, oxidation resistant, and could be formed at lower $\mathrm{pHs}$, thanks to the lower $\mathrm{p} K_{\mathrm{a}}$ of nitrocatechols, compared to that of (pyro)catechol. On the other hand, complexation of Fe"l by nitrocatechols was very poor. With regard to catechol derivatives without electronwithdrawing groups, it was shown that they efficiently bind to $\mathrm{Fe}^{\text {III }}$ atoms, but eventually degrade by oxidation of the catecholic moiety, leading to poor final stabilization. Finally, mimosin, a catechol-semiquinone analog, was shown to have such a high affinity toward the NPs that it led to their incipient dissolution. ${ }^{[185]}$ The stability ( $>1 \mathrm{~d}$ ) of iron oxide NPs functionalized with dopamine anchors was also investigated by Carpenter et al. It was concluded that despite their good initial dispersibility in aqueous media, NPs eventually degrade because of oxidation of the dopamine moieties by Fe"II ions on the NP surface. The authors provide a tentative step-wise mechanism explaining the eventual detachment of the oxidized dopamine layer and concomitant precipitation of the NPs. ${ }^{188}$

In order to gain insight on the NP aggregation process, $\mathrm{Fe}_{3} \mathrm{O}_{4}$ nanoparticles were coated with random copolymer brushes of 2-(2-methoxyethoxy) ethyl methacrylate and oligo(ethylene glycol) methacrylate, with molar fractions of $90 \%$ and $10 \%$, respectively, anchored to the surface through dopamine end-caps. The authors showed that nanoparticles had robust colloidal stability and excellent biocompatibility. Plus, the aggregation-disaggregation of the NPs was reversible and adjustable by temperature control. ${ }^{[189,190,191]}$ In a further study, it was shown that nanoparticles thus functionalized had a good balance of interchangeable hydrophobic and hydrophilic characters that might explain their ability to cross cell membranes. ${ }^{[192]}$

Simard and co-workers focused on the protection of pure Fe NPs using a core-shell methodology, in order to obtain nanoparticles with higher magnetization values than those of $\mathrm{Fe}_{\mathrm{x}} \mathrm{O}_{\mathrm{y}}$. A multi-layer approach was used, where a magnetic Fe core was subsequently coated with different metal and metal-oxide shells. In the case of NPs with an exterior crystalline $\mathrm{Fe}_{\mathrm{x}} \mathrm{O}_{\mathrm{y}}$ shell, the last coatings applied were dopamine and dopamine-PEG. Coated nanoparticles showed near-monodispersivity and excellent stability in aqueous media at different $\mathrm{pHs}$, and kept their superparamagnetic behavior at least up to $1 \mathrm{yr}$. Magnetization values were in accordance with the $\mathrm{Fe}$ nucleus $/ \mathrm{Fe}_{x} \mathrm{O}_{\mathrm{y}}$ coating ratio, making them superior in this respect to other particles with non-magnetic (for instance, $\mathrm{Au}$ ) protective coatings. ${ }^{[176]}$

\subsection{Therapeutic uses and imaging.}

One of the first reports on the functionalization of $\mathrm{Fe}_{3} \mathrm{O}_{4}$ nanoparticles for potential therapeutic uses was published by $\mathrm{Xu}$ and co-workers. NPs were coated with a ligand featuring dopamine as an anchor and a terminal porphyrin. The authors suggested that the simultaneous presence of superparamagnetic magnetite cores and porphyrin ends in the same particles was intended as a double strategy for cancer therapy, since both moieties are potentially therapeutic for hyperthermia and photodynamic cancer therapy, respectively. Modified nanoparticles were able to penetrate the cytoplasm of HeLa cells and induce apoptosis upon irradiation with light of a suitable wavelength. ${ }^{[180]}$ The same research group later reported on the design of coated NPs in which functional moieties were located in the NP core, as opposed to the more usual approach by which they are linked to the surface coating. 'Yolk-shell' FePt nanoparticles were thus prepared, where FePt NPs were coated with porous 
shells of iron oxide. NPs were finally surface-modified with L-DOPA for adequate dispersion in aqueous media. In this case, the functional payload consisted of highly cytotoxic Pt ions released from the FePt core once this started to oxidize, and the Pt ions then squeezed through the $\mathrm{Fe}_{2} \mathrm{O}_{3}$ shell. The NPs were shown to be superparamagnetic and suitable for MRI detection. With regard to therapeutic activity, the NPs turned out cytotoxic against HeLa cells, the $\mathrm{IC}_{50}$ values in terms of $\mathrm{Pt}$ mass per unit volume being comparable to those of cis-platin. ${ }^{[181]}$

Messersmith and co-workers reported the coating of $\mathrm{Fe}_{3} \mathrm{O}_{4}$ nanoparticles with dopaminefunctionalized hyaluronic acid. The coating was effective in ensuring good dispersion of the NPs in aqueous media for at least 4 days, after which the quality of the dispersion was found to correlate with the degree of dopamine conjugation. The usefulness of the surface-modified NPs in cancer therapy was tested by studying by MRI the preferential uptake of the NPs by strains of cancerous cells having over-expressed hyaluronic acid receptors. Blank experiments without dopamine showed a low degree of uptake, proving the essential role of the robust catecholic anchorage. On the other hand, hyaluronic acid played a double role, both as NP stabilizer and target moiety. ${ }^{[177]}$ Another selective therapeutic approach was reported by Sun et al., using 4-methylcatechol as anchor for the iron oxide NPs. A subsequent Mannich reaction of the catechol with formaldehyde and an oligopeptide afforded functionalized NP that were shown stable in aqueous suspension for long times and able to target tumor cells in vivo, showing specificity for the target molecule expressed by the tumor cells and little uptake by the immune system. ${ }^{[182]}$

Jiang and co-workers coated $\mathrm{Fe}_{3} \mathrm{O}_{4} \mathrm{NPs}$ with a DOPA$A_{2}$-grafted polycarboxybetaine metacrylate. The magnetic NPs showed long term stability in physiological conditions and lower macrophage uptake compared with dextran-coated NPs. When the coated NPs were further functionalized with a suitable target peptide, they showed enhanced uptake by human umbilical vein endothelial cells, as visualized by MRI. ${ }^{[174]}$

Labeling of nanoparticles with lanthanides for biomedical applications has been reported recently. Park and co-workers functionalized $\mathrm{Fe}_{3} \mathrm{O}_{4}$ NPs with both DOPA-anchored PEG chains -in order to provide aqueous stability- and functional chains, consisting of dopamine anchors covalently linked to a specific chelating agent for $\mathrm{Gd}^{\prime \prime \prime}$. The resulting Gd-labeled magnetite nanoparticles were intended for use as high resolution dual ( $T_{1}$ - and $T_{2}$-weighted) MRI contrast agents. Their performance in vivo showed the enhanced accuracy provided by the dual detection. The authors remarked that the superparamagnetic character of magnetite was not altered significantly upon surface modification. ${ }^{[178]}$ Yang et al. prepared two different

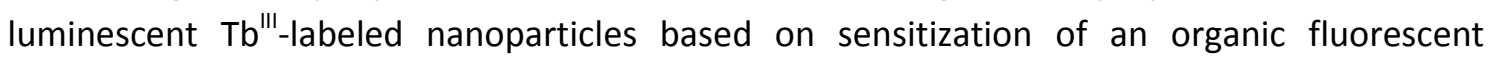

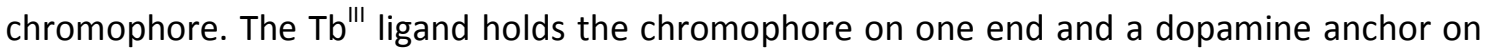
the other. It was estimated that about 2000 Tb cations were associated to each magnetite NP this way. The NPs showed low cytotoxicity, high uptake capacity by HeLa cells and simultaneous superparamegnetic behavior and strong fluorescence needed for dual MRI/fluorescence cell imaging. ${ }^{[186]}$

$\mathrm{Xu}$ and co-workers reported a general methodology for the preparation of nanoparticulate heterodimers with sizes below $20 \mathrm{~nm}$. The authors showed that each half of the dimer could be functionalized separately owing to the different surface characteristics of the substrate particles and the respective affinities of the ligands to the substrates. The ability to fabricate multifunctional heterodimers was exemplified by fabricating a $\mathrm{Fe}_{3} \mathrm{O}_{4}-\mathrm{Ag}$ nanoparticulate assembly, and subsequently attaching a dopamine-anchored biotin to the $\mathrm{Fe}_{3} \mathrm{O}_{4}$ half, and a 
thiolated porphyrin to the Ag moiety. ${ }^{[193]}$ In a follow-up investigation, the same authors prepared dumbbell-like heterodimers of $\mathrm{Fe}_{3} \mathrm{O}_{4}$-Au nanoparticles with core diameters of $20 \& 8$ $\mathrm{nm}$, respectively. To the magnetite moiety, epidermal growth factor receptor (EGFR) antibody was attached via PEG, using dopamine as an anchor. On the other side, the Au NP was protected with HS-PEG-NH . Dumbbells were stable against aggregation in biological media, showed superparamagnetic behavior on account of the $\mathrm{Fe}_{3} \mathrm{O}_{4}$ moiety, and exhibited surfaceplasmonic absorption thanks to the Au moiety. A431 cells known to over-express the EGFR showed selective absorption of the NPs. This was detected by reflectance at the appropriate wavelength using confocal microscopy, which resulted in stable images even after 6 days. This was considered particularly important, since it would allow long-term tracking of the NPs in cell cultures, as opposed to fluorescent tags, for which signal loss is usually very fast. ${ }^{[194]}$

Recently, Mattoussi and co-workers reported the PEG-functionalization of iron oxide NPs with a novel polymer bearing multiple dopamine moieties. The use of such multidentate anchors afforded a much higher colloidal stability of the NPs, especially across a broad range of $\mathrm{pH}$ and electrolyte concentrations, as compared with the usual approach consisting of single anchored functional moieties. The modified NPs showed no relevant cytotoxicity, could be used as MRI contrast agents, and were amenable to the incorporation of additional functionalities through appropriate chemical modification of PEG chains. ${ }^{195}$

\section{3. (Bio)sensors.}

In one of the first studies showcasing catechol-as-anchor functionalization of NPs, Xu and coworkers reported the synthesis of a composite material comprising magnetic nanoparticles $\left(\mathrm{Fe}_{2} \mathrm{O}_{3}, \mathrm{SmCO}_{5}\right)$, dopamine as anchor moiety for a nitrilotriacetic acid- $\mathrm{Ni}^{2+}$ complex, intended as a selective chelating agent for hystidine-tagged proteins. It was shown that this ensemble is able to bind the proteins of interest with higher selectivity than a commercial affinity column. The functionalized nanoparticles were stable, and could be regenerated and reused for the separation purposes mentioned. ${ }^{[196]}$

More recently, Yang, Sun and co-workers reported that rhodamine 6G (Rh6G) coupled to PEGdopamine-modified $\mathrm{Fe}_{3} \mathrm{O}_{4}$ nanoparticles could be used as an $\mathrm{Fe}^{\mathrm{III}}$-selective fluorescent sensor. It was further shown that functionalized NPs may detect Fe" inside cells, by incubation of HeLa cells in Fe"'-containing biological media and final uptake of the NPs. A strong red fluorescence

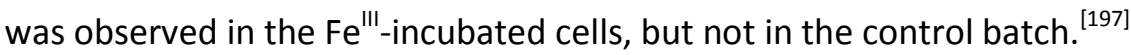

An early report by Rajh and co-workers on DNA recognition described the preparation of triadic systems comprising $\mathrm{TiO}_{2}$ nanoparticles, dopamine anchors and DNA oligonucleotides. With DNA sequences having the proper oxidation potential and upon the action of light, electrons could be transferred from the DNA strand to the dopamine- $\mathrm{TiO}_{2}$ dyad, thanks to the efficient electronic coupling of the dopamine anchor to the $\mathrm{TiO}_{2}$ surface. The charge separation was so extended that resulting charges did not recombine and were, thus, available for redox reactions at both $\left(\mathrm{DNA} / \mathrm{TiO}_{2}\right)$ ends of the system. Importantly, the redox properties of the triad were found dependent on the oligonucleotide sequence of the DNA, as well as on the extent of mismatch between NP-bound DNA strands and complementary "sensed" strands to which it hybridized. ${ }^{[198]}$ In a follow-up investigation, it was found that upon the action of light, electrons were transferred from DNA to the dopamine- $\mathrm{TiO}_{2}$ dyad, whereas holes were transferred selectively to guanine bases. The efficiency of the charge separation was dependent upon the distance of the first guanine base (hole trap) and the existence of adenine bases (hopping 
sites). This effect was studied photochemically, and also by observing the amount of $\mathrm{Ag}$ deposited by the photochemically induced electron transfer to the electrode: The more efficient the ET, the higher the amount of charge transferred to the Ag+ solution and thus the greater the amount of $\mathrm{Ag}$ precipitated. ${ }^{[199]}$

Recently, Park et al. reported the use of dopamine-anchored fluorescein-grafted hyaluronic acid to gold nanoparticles as fluorescent labels. Upon incubation of the NPs in test media where reactive oxygen species (ROS) were being generated, hyaluronic acid degraded, eventually liberating the fluorescein moiety and thus increasing the overall fluorescence, which stayed low for as long as the polymer chains remained attached to the gold NPs (quencher). These fluorescent-tagged NPs were suggested as real-time markers for ROS, with a fluorescent signal higher than that of commercial fluorescent probes for both chemically- and intracellular-generated ROS. ${ }^{[200]}$

Wang et al. reported coating magnetite NPs with polydopamine, on which Au NPs were later grown. This nanocomposite material was used to make an electrochemical sensor for hemoglobin and interleukin-6. ${ }^{[201]}$ In two separate studies, molecular imprinting analysis of proteins was explored by coating iron oxide NPs with polydopamine in the presence of myoglobin, horse-radish peroxidase, ${ }^{[202]}$ and bovine hemoglobin. ${ }^{[203]}$ The imprinted coating was shown to recognize the template proteins with acceptable selectivity. Furthermore, the adsorbed proteins could be easily isolated from the solution by using a magnet. ${ }^{[203]}$ In another study, Zhang and co-workers prepared polydopamine-coated carboxyl-functionalized magnetite NPs for protein recognition. ${ }^{204}$

\subsection{Miscellaneous NP functionalization.}

A few studies have recently focused on controlled release of active species involving catecholic moieties. Sun and co-workers coupled 6-hydroxy-chromone-3-carbaldehyde -used as a model drug with antifungal, antiviral, antihypertensive, and anticancer activity-, to PEG-dopamine$\mathrm{Fe}_{3} \mathrm{O}_{4}$ nanoparticles via Schiff-base chemistry. This way, the drug could be set free by adjustment of the $\mathrm{pH}$ by hydrolysis of the Schiff-base bond, indicating that these NP conjugates may be thought of as control-release systems in acidic environments. In vitro tests showed that drug conjugates were soluble in the biological culture medium and readily absorbed by HeLa cells, were they presented a high level of cytotoxicity. ${ }^{[183]}$ An interesting architectural strategy involving magnetic NPs and controlled release was reported by Reinhult and co-workers. The use of coatings based on hydrophobic chains, instead of PEG, afforded palmityl-nitroDOPA-coated NPs able to spontaneously associate with a specific phospholipid capable of forming micelles. One self-assembled, the liposomes were shown to carry the NPs embedded in the lipidic bilayer. This structure was responsive to the application alternating magnetic fields, which upon heating the NPs, prompted the dissociation of the lipidic wall and the release of a proof-of-concept cargo -a fluorescent probe- ${ }^{[184]}$

$\mathrm{Fe}_{3} \mathrm{O}_{4}$ NPs were used as controllable nano-scavengers using dopamine-anchored bis(phosphonate) groups to scavenge uranyl cations from water and blood samples. Uranyl removal was reported to be quantitative in contaminated water samples, and as high as $69 \%$ in blood samples where phosphate ion in solution and proteins compete for uranyl coordination. After complexation, nanoparticles were easily removed from the medium with a magnet. As of 
the publication date, this was the first reported example of removal of radionuclides from biological fluids using nanoparticles. ${ }^{[179]}$

Catecholic anchors have allowed the incorporation of iron oxide nanoparticles into complex frameworks and the adoption of sophisticated coating methodologies. Tremel and co-workers reported coating $\mathrm{Fe}_{2} \mathrm{O}_{3}$ nanocrystals with $\mathrm{SiO}_{2}$ shells using an enzymatic strategy. NPs were coated with a polymer containing a dopamine anchor at one end, and a nitrilotriacetic acid moiety at the other, the latter capable of binding $\mathrm{Ni}^{\prime \prime}$. In a second stage, $\mathrm{Ni}^{\prime \prime}$ cations were bound to the surface of the coated NPs. The coordination sphere of the $\mathrm{Ni}^{\prime \prime}$ centers was then completed with hystidine moieties via coordination by a hystidine-tagged silicatein. Finally, upon addition of tetraethoxysilane to a dispersion of the modified nanoparticles, silicatein catalyzed the formation of a silica crust around them. ${ }^{[170]} \mathrm{Xu}$ et al. prepared a magnetorheological hydrogel as a composite material fabricated by mixing a conjugate of 2(naphthalen-2-yl) acetic acid with Phe(nylalanine)-Phe(nylalanine) and $\mathrm{Fe}_{3} \mathrm{O}_{4}$ nanoparticles surface-functionalized with a Phe-Phe-catechol conjugate. The first molecule was able to form a hydrogel by itself, which on mixing with the functionalized magnetite NPs, spontaneously incorporated them into a nanofiber network. Upon application of even small magnetic fields, the hydrogel became liquid and the nanoparticles precipitated out. Heating/cooling cycles were able to reversibly regenerate the hydrogel. ${ }^{[171]}$ Catecholic anchors also facilitated the incorporation of liquid crystals and magnetic NPs in polymeric matrices. A polysiloxane backbone copolymerized with dopamine metacrylate and nematic liquid crystal mesogens was further reacted with ferrite nanoparticles, which end up attached to the polymer through the catecholic pendant groups. $1 \mu \mathrm{m}$-thick films of the $\mathrm{Fe}_{3} \mathrm{O}_{4}$-modified polymer on Indium-doped tin oxide (ITO) surfaces were shown to be transparent in the visible range. The degree of transparency, directly related to the phase order, could be tuned by the application of a magnetic field. ${ }^{[172]}$

With the exception of DSSC-related applications (Section 8.2), and quantum dots (Section 8.3) a brief review of the functionalization of non-ferric NPs intended for use in materials applications will follow.

Studart and co-workers prepared coatings for $\mathrm{Al}_{2} \mathrm{O}_{3}$ nanoparticles that were highly efficient against self-aggregation by depositing a layer of gallol-PEG brushes of tailored length onto 65$\mathrm{nm}$ alumina nanoparticles, making it possible to obtain aqueous suspensions of the nanoparticles of up to $40 \& \mathrm{w} / \mathrm{v} \cdot{ }^{[205]}$ Carbon nanotubes coated with polydopamine were thus made water-dispersible and functionalizable, for example, by reductive precipitation of Au NPs from a solution of $\mathrm{HAuCl}_{4}$. The polydopamine coating did not alter the morphology of the nanotube walls. ${ }^{[206]}$ Protonated dopamine was used as both anchor and surface modifier for NPs employed in cathodic electrophoretic deposition (EPD) methods used to fabricate supercapacitors. $\mathrm{MnO}_{2}$ nanoparticles and multi-walled carbon nanotubes (MWCNTs) thus coated were found effective to obtain stable suspensions. Different binding mechanisms for the anchors were presumed: ligand-metal coordination for $\mathrm{MnO}_{2} ; \pi-\pi$ stacking for the MWCNTs. ${ }^{[207]}$ A similar strategy was applied to coat $\mathrm{ZnO}$ and $\mathrm{TiO}_{2} \mathrm{NPs}$ for the electrophoretic deposition of these in composite films. ${ }^{[208]}$

Messersmith and co-workers reported a method to anchor polymethylmetacrylate chains to a $\mathrm{TiO}_{2}$ surface, using a dopamine derivative both as anchoring moiety and initiator for SI-ATRP polymerization. The brominated dopamine derivative was first attached to the $\mathrm{TiO}_{2}$ surface and polymeric PMMA chains were later grown on the anchor. Because of the anchoring 
abilities of dopamine to a variety of substrates, this method was intended as a generalpurpose way of functionalizing all kinds of surfaces by ATRP. [209]

In their study of nitrilotriacetic acid (NTA)-based chelating agents, Tremel and co-workers prepared a dual-anchor polymeric backbone functionalized with grafted NTA and dopamine pendant groups. NTA anchors were specifically chosen to partially coordinate $\mathrm{Ni}$; the rest of the coordination sphere of this atom was then coordinated to surface $S$ atoms forming part of the external layer of $\mathrm{MoS}_{2}$ nanoparticles or nanowires (termed, respectively, inorganic fullerenes and nanotubes). As opposed to unmodified $\mathrm{MoS}_{2}$, coated $\mathrm{MoS}_{2} \mathrm{NPs}$ were soluble in polar solvents. Finally, through the available catecholic anchors, coated $\mathrm{MoS}_{2}$ NPs were attached to $\mathrm{TiO}_{2}$ nanorods, opening up the possibility to synthesize hybrid systems containing $\mathrm{MoS}_{2}$ nanoparticles and surfaces with other functional properties. ${ }^{[210]}$

$\mathrm{TiO}_{2}$ nanorods could be organized into liquid crystal phases after modification of the insoluble nanorods by means of a block copolymer comprising catecholic anchoring blocks and two different metacrylate esters as soluble blocks. Once functionalized, the nanorods were soluble and showed ability to pack densely together and form nematic and smectic mesophases, in which order was achieved by applying shear and meniscus forces. ${ }^{[211,212]}$ When ZnO nanorod polymer hybrids with polystyrene soluble blocks were prepared, nanorod mesogens were observed to switch their preferential orientation under an applied AC electric field, from planar (parallel to the substrate) to homeotropic (perpendicular) in full analogy to the switching of low molecular weight liquid crystals inside an electric field. ${ }^{\text {[213] }}$

A few studies describe catechol-based coated NPs in potential catalytic applications. As part of their investigation on surface modification of $\mathrm{TiO}_{2}$ nanoparticles with enediol ligands, Dimitrijevic et al. reported that 3,4-dihydroxyphenylacetic acid and dopamine coatings modify the electrochemical overpotential of hydrogen gas evolution on $\mathrm{TiO}_{2}$ nanoparticulate films. ${ }^{\text {[214] }}$ In a parallel study, Rajh and co-workers coated with dopamine $\mathrm{TiO}_{2}$ nanoparticles deposited on an ITO electrode. This coating was subsequently functionalized with biotin, to which avidin was complexed. The authors showed that avidin was able to accept photoinduced holes through the dopamine-biotin system, which led to dissociation of the biotin-avidin complex, probably because of the oxidation of the Tyr and Trp residues in avidin. This, in turn, led to a decrease in the photoinduced current intensity. ${ }^{215]}$

The enhancement of polymer-filler interactions in composite materials is highly desirable in order to improve the mechanical properties of the polymer matrix, as exemplified by silicasilane coupling in silica-filled elastomeric matrices. ${ }^{[216]}$ Because of its affinity to a variety of inorganic substrates, catechol-based coatings offer an alternative to existent techniques for filler modification. Pan and co-workers reported the preparation of a co-styrene-butadiene (SBR) elastomer terminated with 3,4-dihydroxybenzaldehyde (DOBA). The catecholic end group was chosen because of its strong affinity to both hydrophobic carbon black and hydrophilic inorganic oxides $\left(\mathrm{SiO}_{2}, \mathrm{TiO}_{2}\right)$, which constitute some the most widely used fillers for elastomeric materials. Compared with a reference unmodified SBR co-polymer, DOBAmodified SBR showed a significant improvement in its mechanical properties, such as tensile strength, when reinforced with carbon black and $\mathrm{TiO}_{2}$. As far as viscoelastic properties are concerned, DOBA-modified SBR composites showed novel, interesting behavior. ${ }^{[217]}$ Applying a complementary strategy, Lu et al. modified a clay filler with polydopamine. It was found that the strong interfacial interactions brought by the polydopamine benefited not only the dispersion of the D-clay in an epoxy resin, but also the effective interfacial stress transfer, 
leading to greatly improved thermomechanical properties at very low filler loadings. ${ }^{[218]}$ In a similar fashion, Wang et al. rendered a carbon black filler hydrophilic and water dispersible by coating it with polydopamine. ${ }^{219}$ Taking advantage of the distinct chemical nature of each lamellar face in mineral kaolinite -with tetrahedral silicon oxide groups on one side, and octahedral aluminum oxide/hydroxide layers on the opposite-, Breu and co-workers were able to selectively functionalize aluminum faces with a hydrophobic catechol derivative, while substituting $\left[\mathrm{Ru}(\mathrm{bpy})_{3}\right]^{2+}$ for $\mathrm{Na}^{+}$cations on the silicate-layered side. This way, the incipient Janus-like character of native kaolinite could be remarkably enhanced to afford, in this particular case, an amphiphilic variety of kaolinite. ${ }^{220}$ In another study by Bourmaud and coworkers, polydopamine was used to treat polylactic acid in order to increase its compatibility with hemp fibers; these modified composites showed improved adhesion and mechanical properties. $^{[221]}$

Choi and co-workers reported the coating of single cells with a $\mathrm{TiO}_{2}$ shell of nanometer thickness, which was shown not impair cell viability significantly. Furthermore, the titania shell was amenable to functionalization via catechol chemistry, as exemplified by surface absorption of pyrocatechol violet dye. ${ }^{222}$ 


\section{Functional scaffolds.}

\subsection{Oriental lacquers.}

Oriental lacquers (urushi) are manmade coatings of natural origin sourced from the cured sap of several trees native to Southeast Asia, -particularly, Rhus verniciflua-, that have been used for more than 2,000 years across the region to decorate objects with a flawless, high-gloss, polished finish. In addition to their highly artistic and cultural relevance, oriental lacquers have been studied for their useful qualities, such as being biodegradable, eco-friendly surface protectors, ${ }^{[223]}$ with better solvent resistance compared to synthetic substitute lacquers, and excellent durability when stored in proper conditions. ${ }^{[224]}$ Regarding its chemical composition, the sap of the lacquer tree is an o/w emulsion of several long-chain $\left(C_{15}, C_{17}\right), 3$ - and 4- monoand polyunsaturated alkylcatechols -collectively known as urushiol-, in an aqueous medium containing a complex mixture of polysaccharides, glycoproteins, metal ions and several enzymes with phenoloxidase activity, of which laccase is recognized as the most important one. ${ }^{[225]}$ It is worth mentioning that urushiol-type alkylcatechols are not unique to lacquer trees; they are indeed secreted by several other plant species of the Anacardiaceae family, especially of the Toxicodendron genus, such as poison oak and poison ivy, where these chemicals are responsible for causing a notorious contact allergy. ${ }^{[226]}$ Compared with poison ivy, urushiol, lacquer tree sap is richer in tris-unsaturated alkylcatechols, in which multiple insaturations facilitate aerobic polymerization reactions catalyzed by accompanying enzymes. ${ }^{[227]}$ This polymerization reaction, which kills the allergenic activity of the urushiol monomers, is rather complex, involving several kinds of oxidative reactions, including direct ring-ring and ring-side chain double bond couplings, and side chain double bond oxidations and couplings. Although most new bonds are $\mathrm{C}-\mathrm{C}, \mathrm{C}-\mathrm{O}$ bonds between linked units have been also observed. ${ }^{[224,228]}$

The fabrication of a traditional urushiol lacquer is a slow and laborious process resembling covalent multilayer assembly, ${ }^{[229]}$ which relies on the enzymatic curing action of laccase. Typically, the sap is applied to the surface as a solvent-less varnish, under controlled temperature and relative humidity conditions; ${ }^{[225]}$ once dry, the lacquer layer is carefully polished prior to the application of next coating layer. Up to 30-40 layers of 5-10 $\mu \mathrm{m}$ each are required for a quality lacquer coating. ${ }^{[227]}$ Since the long overall duration of the traditional process is a major drawback, attempts have been made at developing alternative, more expedient curing methods, ${ }^{[230]}$ without impairment of the visual qualities and eco-friendliness of traditionally-cured lacquers. Miyakoshi et al. reported that the addition of aminoterminated silanes to urushiol yielded a fast curing urushiol-silane hybrid. ${ }^{[231]} \mathrm{Hu}$ and coworkers found that extracted urushiol may be photo-cured in 2 min by irradiation with UV light, affording a wrinkled material. ${ }^{[232]}$ Optimization of the irradiation conditions improved the finish quality of the UV-cured lacquer, which showed better chemical and solvent resistance than laccase-cured ones. ${ }^{[223]}$ When urushiol was hydrogenated to almost quantitative saturation of the alkyl side chains, a sufficiently fast photopolymerization could be carried out while still obtaining glossy surfaces of sufficient quality to be usable for the restoration of lacquered antiques. ${ }^{[233]}$ 
Lin et al. reported the polymerization of urushiols with UV light in the presence of transition metals, such as Cu". This improved the thermal stability of the lacquer by TG analysis, likely due to coordination between the catechol moieties and the metal center. ${ }^{[234]}$ Urushiol composites with micro- and nanoparticles have been recently reported by the same authors. When urushiol was polymerized in the presence of $\mathrm{Ag}^{\prime}$, the authors observed the precipitation of $\mathrm{Ag}$ microparticles and a sharp increase in the surface conductivity of the composite material. ${ }^{[235]}$ Composite polyurushiol materials with homogeneously dispersed PbS nanorods, ${ }^{[236]}$ and CdS nanoparticles, ${ }^{[237]}$ were obtained by in situ UV-polymerization of urushiol/metal acetate mixtures and further exposure of urushiol-metal films to thioacetamide solutions. Urushiol was also used to coat silica nanoparticles by simple grafting of the catecholic moieties to the polar surface of the NPs, ${ }^{[238]}$ and to prepare fluorescent coatings of a urushiol/bis-hydroxyquinoline-Al'" complex, by growing nanospheres of this material on glass using a "reverse" breath figure method. ${ }^{[239]}$

Hybrid co-polymers of urushiol and polyurethane have been prepared by Cheong and coworkers. Although the thermal stability of the copolymer slightly decreased with increasing urushiol content, the latter had a beneficial effect in corrosion tests and, especially, in showing antibacterial activity against S. aureus. ${ }^{[240]}$ Multilayer films of polyurushiol (PU) and polyvinylpyrrolidone (PVP) were prepared by Weng et al. by alternatively dipping quartz and $\mathrm{CaF}_{2}$ substrates in solutions of PVP and urushiol, followed by UV-curing. AFM and hardness measurements indicated that UV-curing induced a certain degree of layer interpenetration. ${ }^{[241]}$ When this process was carried out using urushiol/Cul solutions instead, PU/Cu microparticles embedded in PVP were observed to form on a pre-cured PU/PVP surface. Final treatment of the patterned substrates with a $\mathrm{Na}_{2} \mathrm{~S}$ solution induced the growth of smaller CuS nanoparticles on the surface of the microparticles, yielding a superhydrophobic coating. ${ }^{[42]}$

The natural polymerization process of urushiol has inspired Kobayashi and co-workers the synthesis of so-called "artificial urushi", in which synthetic analogues of urushiol -both catechol- and phenol-based- were polymerized in mild aqueous conditions using laccase, to obtain hard, high-gloss films with excellent dynamo-mechanical properties. ${ }^{[199,243]}$ This strategy was expanded to use iron(II)-N,N'-ethylenebis(salicylideneamine) as a substitute for a peroxidase enzyme in the "green" polymerization of alkylsubstituted phenols obtained from renewable sources. ${ }^{[199,244]}$ A semi-synthetic analogue of urushiol was prepared by Lu, Zhang and co-workers by condensing natural urushiol, aniline and formaldehyde. The resulting thermosetting resin, an urushiol-based polybenzoxazine, showed excellent toughness, with lower tensile strength, but higher elongation at break than a typical polybenzoxazine, and comparable thermal resistance. ${ }^{245}$

\subsection{Melanin.}

Melanin is a generic term used to describe a variety of biopolymers found widespread in nature as pigments -including those found in hair, skin, eyes, feathers and Sepia ink-, derived from a common catecholic precursor -3,4-dihydroxyphenylalanine or DOPA- through a complex biosynthetic route known as melanogenesis. ${ }^{[246]}$ The most widely studied type of 
melanin is dark-brown eumelanin, ${ }^{[247]}$ recognized for its essential role as photoprotector. ${ }^{[248]}$ Chemically speaking, eumelanin is a macromolecular structure formally constituted of 5,6dihydroxyindole (DHI) and 5,6-dihydroxyindolecarboxylic acid (DHICA) units in varying amounts, $^{[247]}$ linked together in potentially different configurations. The first stages of melanogenesis -i.e., those prior to polymerization- were already outlined by Raper, ${ }^{[249]}$ and Mason, ${ }^{[250]}$ and although later refined by subsequent investigations, ${ }^{[251]}$ are still generically accepted. In essence, they involve a cascade of oxidation, reductive addition and tautomerization steps, some of them enzymatically controlled, featuring high-reactivity 0 quinones as electrophiles and oxidizers, and catecholic molecules as reducing species. ${ }^{[26]}$ Even though extensive research has been carried out on their structure, melanins are among the few last biomacromolecular systems for which the organization of their constituent molecules, and its relation to precise biological functions, are still subject of debate, no less because of melanin's notorious lack of solubility in all solvents, which makes its chemistry particularly challenging. ${ }^{[252,253,254]}$ On the other hand, melanins present a remarkably broad set of physicochemical properties, remarkable for their potential role as functional materials, such as antioxidant and free radical scavenging behaviour, ${ }^{[255]}$ broad-band monotonic absorption in the UV-Vis range, ultrafast thermal relaxation, ${ }^{[256]}$ concomitant with extremely low radiative quantum yield, ${ }^{[257]}$ and interesting electrical properties, such as reversible electrical switching, ${ }^{[258]}$ and electrical conductivities typical of semiconductors, ranging from $10^{-5}$ to $10^{-13}$ $\mathrm{S} \cdot \mathrm{cm}^{-1}$, which are strongly dependant on relative humidity.

In the past years, significant advances have been done towards the elucidation of eumelanin structure. Indeed, the question of whether melanin is formed by high-molecular weight polymeric chains or aggregates of oligomers (protomolecules) is still not settled, although recent spectroscopic evidence seems to favor the latter theory. ${ }^{[259,252]}$ Watt and co-workers used low-voltage HRTEM to show that both synthetic and natural eumelanin consists of nanoaggregates of planar sheets of different sizes, separated by 3.7-4.0 ̊ spacings, typical of $\pi-\pi$ stackings in heteroaromatic systems. Moreover, the unique spectroscopic properties of melanin were found to be independent of aggregation, therefore indicating that they may be explained mostly by its primary structure. ${ }^{[252]}$ This evidence seems to support a 'chemical disorder' hypothesis recently put forward by Meredith and co-workers, ${ }^{[260]}$ according to which eumelanin would consist of a heterogeneous ensemble of chemically distinct chromophores, each with different photo-excitation energies. Individual chromophoric moieties would include colorless, reduced catecholic units (DHI, DHICA) and their respective colored oxidation products (indolequinones and related tautomeric forms), present in different proportions and linked together in different configurations. In addition to catechols and quinones, radical semiquinoid species in comproportionation equilibrium with these would be present in small amounts, accounting for the experimental observation of persistent ESR signals. The existence of stable amounts of quinones and quinoid radicals -which would otherwise be very shortlived- in eumelanin may be justified by steric hindrance within the macromolecular scaffold. ${ }^{[253]}$ Kaxiras and co-workers reported DFT in vacuo calculations on model cyclic, porphyrin-like, planar tetramers arising from all possible combinations of DHI, DHI-quinone and its tautomers -DHI-quinoneimine and DHI-quinone methide-. Results showed that hybrid cyclic frameworks containing both DHI-quinones and DHI moieties would have sufficiently low energy as to co-exist with the lowest-energy, fully DHI tetramer. Remarkably, the calculated UV-Vis spectrum of averaged mixtures of tetrameric molcules would indeed reproduce the monotonic absorption of eumelanin in this range, ${ }^{[261]}$ and its characteristic, ultrafast thermal 
relaxation upon photoexcitation. ${ }^{[262]}$ On the other hand, calculations also showed that stacked aggregates of these porphyrin-like rings would feature interplane distances consistent with those obtained from X-ray diffraction of eumelanin. Overall, these calculations would both support the chemical disorder hypothesis, and a stuctural framework made of oligomeric structures, ${ }^{[261]}$ although covalently-linked oligomers, either stacked, ${ }^{[263]}$ or in helical configurations, might also be feasible. ${ }^{[264]}$ On the experimental side, investigations on soluble synthetic analogues of melanin by Pezzella et al. suggested that the 'chemical disorder' model would be feasible, with co-existing catecholic and quinoid moieties in dynamic equilibrium and quinones accounting for the dark coloration, notwithstanding light scattering effects. ${ }^{[265]}$ On the other hand, neuromelanin has been described as composed of granules around $30-\mathrm{nm}$ diameter consisting of pheomelanin at the core and eumelanin at the surface. ${ }^{[266]}$

In recent years, the most widely researched synthetic analogue of melanin has likely been Messersmith's polydopamine, ${ }^{[72]}$ reviewed in Section 2, to which the reader is referred. Suffice it to say here that, in addition to a similar chemical base, polydopamine and eumelanin share many characteristic traits, such as a deep dark colour, similar FT-IR, ESR, ${ }^{[267]}$ and XPS spectra, ${ }^{[268]}$ and a similar structure of $\pi-\pi$ stacked aggregates, ${ }^{[269]}$ and the coexistence of catecholic and quinoid units in the same macromolecular scaffold. ${ }^{[260,72]}$ Indeed, synthetic melanin may be prepared by autooxidation of DOPA in mild alkaline aqueous media, ${ }^{[270]}$ in conditions similar to those used for the preparation of polydopamine from structurally related dopamine. ${ }^{[72]}$

Another important feature of melanins, which is mentioned in Section 6, is its siderophore-like ability to scavenge heavy metals in vivo, as observed, for example, in several melanized funghi. ${ }^{[271,272]}$ In humans, iron-melanin binding in brain's substantia nigra, likely resulting in intraneuronal production of free radicals, has been linked to the pathogenesis of Parkinson's disease. ${ }^{[273,274]}$ Since, in general, pigmented tissues present a significant amount of metal ions in vivo, the role of melanin as chelating agent and its metal-binding capacity have been subject to extensive research. ${ }^{[275]}$ On the other hand, the in vitro chelating affinity of melanin for a variety of metal cations was already studied by Sealy and co-workers, ${ }^{[270]}$ who attributed this versatility to the simultaneous presence of several functional groups in the molecule. Although the indolic nitrogen and the carboxylate group (in DHICA) participate in the binding ability of $\mathrm{DHI}$ to metal centers, catechols were recognized as essential for binding at neutral-basic $\mathrm{pHs}^{[247]}$

As recently pointed out by $d^{\prime}$ Ischia and co-workers, ${ }^{[253]}$ melanin may be considered a multifunctional material in which catechol units play an essential role. While recognizing the significant potential of eumelanin as a functional material, the authors conceded that control over the aggregation state of melanins, and hence over its solubility, ${ }^{[265,276,273]}$ should be essential for future developments in this field. Meanwhile, Meredith and co-workers have reported the preparation of free-standing melanin films by electrochemical oxidation of DOPA, ${ }^{[277,278]}$ and device-quality thin films of synthetic melanin, ${ }^{[279]}$ by dissolution of synthetic eumelanin in concentrated ammonia, spin casting on glass and silicon substrates and final evaporation of the solvent/reagent; cast films showed characteristic properties of eumelanin, such as similar UV-Vis absorption profiles and an electrical conductivity of $2.5 \cdot 10^{-5} \mathrm{~S} \cdot \mathrm{cm}^{-1}$ at room temperature and $100 \%$ relative humidity. Furthermore, synthetic melanin films presented excellent thermal stability and resistance to photobleaching. The electrocatalytic 
and magnetic properties of ultrathin nanostructured iron-melanin films have also been studied, showing catalytic activity for the electroreduction of hydrogen peroxide in alkaline and neutral solutions. ${ }^{[280]}$ Another significant step towards the integration of melanin-based biopolymers into devices, has been the synthesis of a hybrid melanin/silicon interface, which shows interesting electronic properties and enables insight into electrical transport mechanisms operating, which have been found to be linked to the melanin hydration state. ${ }^{[281]}$

A composite of chitin and melanin has been recently used in order to successfully reproduce the nano-scale antireflection structure of the black scales in the Troides aeacus butterfly wing, which can be viewed as a natural solar collector. The antireflection behaviours of the black scales were measured and modeled, showing that the antireflection property is caused by the coupled effect of ridges and nano-hole arrays of the scales; ${ }^{[282]}$ the periodically aligned inverse$V$ type ridges transfer light beams to the nano-hole area where the light-trapping effct occurs. Both natural and synthetic melanins have been also described as thermooxidative stabilizers of polymers, increasing the decomposition temperature for poly(methylmethacrylate) by about $50-90 \stackrel{\circ}{ }$. $^{[283]}$

\subsection{Catechol-based Nanoparticles.}

Polydopamine can be prepared as nanocapsules ${ }^{[284,285]}$ and nanoparticles, ${ }^{[267]}$ in the latter case resembling eumelanin particles that constitute Sepia ink. Particularly, polydopamine nanocapsules have been prepared using several different template strategies. In a recent study, the Zhou group reported the deposition of polydopamine on $\mathrm{SiO}_{2}$ nanoparticles and further dissolution of the silica core with HF. Shell thickness could be controlled by the number of repeated coating cycles, which was convenient since it allowed the preparation of sufficiently robust capsules that didn't crack upon drying. On the other hand, capsules were permeable, and able to uptake rhodamine $6 \mathrm{G}$ (Rh6G) in aqueous solutions, especially at high $\mathrm{pHs}$, while releasing the dye in ethanol. ${ }^{[284]}$ In a follow-up study, the $\mathrm{pH}$-controlled uptake and release of three dyes with acid-base activity (methyl orange -MO-, alizarin red and Rh6G) by polydopamine microcapsules, fabricated by coating of polystyrene microspheres followed by dissolution of the PS core by THF, was studied. MO and alizarin red were selectively uploaded at acid $\mathrm{pH}$, whereas Rh6G was uptaken at basic pHs, in accordance with the surface charge of polydopamine and the net charge on each dye molecule, each $\mathrm{pH}$ dependent. Controlled release was observed in reversed $\mathrm{pH}$ conditions. ${ }^{[286]}$ An alternative method reported by Caruso et al. described the preparation of dopamine nanocapsules by o/w emulsification, stabilized by partial condensation of dimethyldiethoxysilane (DMDES). The function of this polymer was to entrap insoluble payload and to serve as template for the polymerization of an outer dopamine layer. Later on, the DMDES core was solubilized in ethanol, leaving the cargo entrapped inside the polydopamine shell. This strategy made possible the fabrication of nanoand microcapsules with a high degree of monodispersivity. As proof-of-concept of this encapsulation strategy, functional components, such as magnetic $\mathrm{Fe}_{3} \mathrm{O}_{4}$ nanoparticles, fluorescent QDs (CdSe/CdS), and an anti-cancer drug (thiocoraline), were successfully encapsulated. ${ }^{[285]}$ Jiang and co-workers reported the sequential incorporation of three different enzymes inside, within, an onto polydopamine microcapsules, respectively. The first enzyme (glucosidase) was encapsulated inside the microcapsule after dopamine was polymerized in the presence of this enzyme and onto polystyrene sulfonate (PSS)-modified $\mathrm{CaCO}_{3} 3 \mu \mathrm{m}$-microparticles, and the $\mathrm{CaCO}_{3}$ inner core was later dissolved with EDTA. A second 
coating of polydopamine was carried out in the presence of a second enzyme (ß-amylase), affording the entrapment of the enzyme within the polydopamine shell. Finally, treatment of the polydopamine capsules with $\alpha$-amylase led to the adsorption of this enzyme on the surface of the microcapsules. ${ }^{[287]}$ In a follow-up study, microcapsules with hybrid inorganic/organic shells were obtained by coating PSS-modified $\mathrm{CaCO}_{3}$ with a first layer of $\mathrm{TiO}_{2}$ or $\mathrm{SiO}_{2}$ precipitated from soluble precursors in the presence of protamine, and subsequent deposition of polydopamine. The inorganic, biomineralized inner crust was shown to stabilize the organic outer layer, particularly in the case of $\mathrm{TiO}_{2}$, thanks to the strength of catechol-Ti bonds. The porosity and thickness of the capsules could be controlled by careful choice of reagent concentrations and coating conditions. ${ }^{[288]}$

Recently, Lee and co-workers reported a simple procedure for the generation of melanin-like nanoparticles by oxidative polymerization of dopamine in basic aqueous media and vigorous stirring. By careful control of the reaction conditions, $<100 \mathrm{~nm}$ nanoparticles of polydopamine were obtained, which were more monodisperse than melanin nanoparticles obtained from Sepia ink (100-300 nm). Spectroscopic data of the synthetic nanoparticles were very similar to those of the natural Sepia eumelanin, including the observation of an ESR signal arising from a certain amount of free radicals in both -synthetic and natural- polymers. The surface of synthetic melanin NPs could be modified by adding a thiol-capped-PEG, which made the NPs dispersible in aqueous media. NPs were found to be non-toxic toward HeLa cells, and showed radical scavenging capacity, which increased with decreasing NP size. For the smallest NPs $(<70$ $\mathrm{nm})$, this capacity was shown to be comparable to that of ascorbic acid. ${ }^{[267]}$

Inspired by biomimetic analogues of Mefps, Deming and co-workers prepared DOPAcontaining polypeptidic block copolymers that proved to self-assemble into nanometer-sized vesicles. In a similar way to Mefps, DOPA moieties cross-link the peptidic shell upon addition of an oxidizing agent $\left(\mathrm{NaIO}_{4}\right)$, yielding very stable vesicles, which were resistant against organic solvents, high-osmotic pressure aqueous media and temperature, without rupture, and capable of retaining their payload -a fluorescent dye-, without cross-linking having any effect in the amount of encapsulated dye. As opposed to DOPA-containing vesicles, polypeptidic scaffolds without DOPA were disrupted by these environmental stress tests. ${ }^{\text {[289] }}$

\subsection{Assisted fabrication of materials.}

Dopamine and DOPA-bearing polymers have been shown to aid in the crystallization of inorganic materials, such as $\mathrm{TiO}_{2},{ }^{[290]}$ vaterite- $\mathrm{CaCO}_{3}{ }^{[291,292]}$ and noble metals ${ }^{[293]}$ in the form of nanoparticles. Messersmith et al. prepared coated gold and silver NPs by taking advantage of the reducing character of catecholic moieties. A tetraDOPA-PEG molecule was shown to react with $\mathrm{Au}^{\prime \prime \prime}$ and $\mathrm{Ag}^{\prime}$ from aqueous basic solutions to yield the corresponding native elements in $<100 \mathrm{~nm}$ nanoparticles coated with oxidized, cross-linked forms of the parent molecule. These NPs presented plasmonic resonances in accordance with their respective sizes and stability in solution thanks to the protective coating afforded by the tetraDOPA-PEG layer $(<10 \mathrm{~nm}) .{ }^{[293]}$ Applications involving the dopamine-assisted precipitation of $\mathrm{Ag}$ nanoparticles by in situ reduction of $\mathrm{Ag}^{+},{ }^{[77,199,141,146]}$ and $\mathrm{Au} \mathrm{NP}$ from $\mathrm{AuCl}_{4}{ }^{-[101,102,103]}$ are discussed in chapter 2.1 . 
Tremel and co-workers described an easy, robust procedure for the preparation of functionalized, monocrystalline rutile $\mathrm{TiO}_{2}$ nanorods in mild conditions. $\mathrm{TiO}_{2}$ was prepared by aqueous hydrolysis of $\mathrm{TiCl}_{4}$ in the presence of dopamine, followed by aging and hydrothermal treatment at $150{ }^{\circ} \mathrm{C}$. Dopamine, which ended up surface-bound, was shown to control the morphology as well as the crystallinity and the phase selection of $\mathrm{TiO}_{2}$. Moreover, the availability of the amine terminal groups of anchored dopamine afforded further functionalization of the nanorods (e.g. with dye 4-chloro-7-nitrobenzofurazan). ${ }^{[290]}$

In a series of recent studies on the preparation of biocompatible materials, Park et al. showed that calcium carbonate can be precipitated into the unstable vaterite phase in the presence of dopamine. Further incubation of dopamine-stabilized vaterite microparticles in simulated body fluid during $48 \mathrm{~h}$ afforded the conversion of vaterite into carbonated hydroxyapatite, which is biocompatible and very similar to natural bone. Blank experiments without dopamine showed the formation of calcite phases in the same experimental conditions. Due to its stability, calcite didn't extensively dissolve in the same incubation conditions, and thus didn't precipitate as hydroxyapatite. When intermediate amounts of dopamine were used, mixed vaterite/calcite phases were obtained. ${ }^{[291,292]}$ In a follow-up study, the authors reported that dopamine-coated Ti substrates could be coated with hydroxyapatite crystals when incubated in simulated body fluid solutions. Polydopamine was thus shown to be essential as nucleating material for hydroxyapatite and effective as a universal strategy for biomineralization, in which a bone-like material (hydroxyapatite) may be successfully integrated with materials of a varied nature and different compatibility, such as metals, ceramics, organic polymers, and porous materials and fibers, such as cellulose. ${ }^{[294]}$ Similar results were obtained with dopamine-coated carbon nanotubes, which were found to be less cytotoxic for osteoblasts than bare CNTs, opening the possibility of using CNTs for applications in bone-related therapies. ${ }^{[295]}$ In a very recent study, polydopamine is used to coat self-assembled nanowires of diphenylalanine intended as substrates for the growth of hydroxyapatite. The authors suggested that the layered structure of polydopamine directed the growth of hydroxyapatite crystals and of preosteoblasts on top, in a process reminiscent of the biomineralization of collagen. ${ }^{[269]}$ Similarly, Wu et al. reported that polydopamine assisted apatite mineralization when used to improve the biocompatibility of mesoporous silica for bone tissue regeneration, affording better attachment of bone marrow stromal cells and sustained release of dexomethasone. ${ }^{[296]}$

\subsection{Nanovessels.}

Catechols have also been reported for the controlled design of functional, non-polymeric scaffolds. Raymond and co-workers showed that carefully designed ligands, such as $N, N^{\prime}$ bis(2,3-dihydroxybenzoyl)-1,5-diaminonaphthalene, self-assemble via catecholate chelation in aqueous media around certain trivalent ( $\mathrm{Fe}^{\mathrm{II \prime}}, \mathrm{Ga} \mathrm{G}^{\mathrm{II \prime}}, \mathrm{Al}^{\mathrm{III}}, \mathrm{In}^{\mathrm{III}}$ ) and tetravalent cations ( $\mathrm{Ti}^{\mathrm{IV}}, \mathrm{Ge}^{\mathrm{IV}}$ ) capable of octahedral coordination, to form tetrahedral organometallic clusters with a $M_{4} L_{6}$ stoichiometry. ${ }^{[297,298]}$ In these complexes, chiral metal ions occupy the vertices of the tetrahedron, while ligands span the structure's edges, forming stable, non-interconvertible enantiomeric structures of 300-500 $\AA$ able to act as both as protective cages and nanovessels. With an overall electric charge of -12 for the trivalent species, the assembled cages are ideally suited for the encapsulation/protection of cationic species of appropriate size, and have been found to catalyze a variety of chemical reactions and physico-chemical effects on guest molecules, among which, aza-Cope rearrangements, ${ }^{[299,300,301]}$ the activation of $\mathrm{C}-\mathrm{H}$ bonds in 
aldehydes, ${ }^{[302,303]}$ the isomerization of allylic alcohols, ${ }^{[304]}$ the stabilization in basic aqueous media of reactive cationic species, ${ }^{[305,306,307]}$ and the enhancement of the basicity of amines. ${ }^{[308]}$ Reactions that are usually acid-catalyzed, such as the deprotection of acetals ${ }^{[309]}$ and the hydrolysis of orthoformates, ${ }^{[310311]}$ have been realized in basic media by addition of catalytic amounts of the Gal' cluster. ${ }^{[310]}$ Once assembled, the catechol complexes were shown to be robust enough, regardless of the trivalent metal used, so that host exchange was facilitated through minor openings by structural, elastic deformation of the cage, rather than its partial dissociation via edge rupture and re-assemblage. ${ }^{[173]}$ Remarkably, these $M_{4} L_{6}$ complexes, dubbed "nanozymes", ${ }^{[09]}$ have been shown to rely exclusively on electrostatic and hydrophobic interactions for thermodynamic stabilization of protonated guest species, and exhibit enzyme-like Michaelis-Menten kinetics for catalytic hydrolysis reactions taking place inside the host. ${ }^{[310]}$ 


\section{Siderophore-like materials.}

In order to regulate key metabolic routes, most living organisms need to take in iron from the environment, and be able to efficiently and reversibly regulate its metabolic concentration by means of specific transport and storage mechanisms. Because of the very low solubility of Fe $\mathrm{III}^{\prime \prime}$ in a broad range of $\mathrm{pH}$, the bioavailability of $\mathrm{Fe}^{\mathrm{III}}$ is very poor despite its otherwise natural abundance, and specific strategies have evolved to acquire this essential need. In this regard, bacteria secrete powerful chelating agents known as siderophores in order to scavenge Fe III $^{\prime}$ from their surroundings. ${ }^{[312,313,314]}$ In conditions of iron deprivation, the role of siderophores is crucial for the survival and proliferation of microorganisms, especially for infectious bacteria, since hosts become the only immediate source of Fe"'. Not surprisingly, the virulence of certain infectious diseases is related to the efficacy and versatility of the secreted siderophores, and enhanced by a higher availability of physiological iron. ${ }^{[315]}$ On the other hand, in order to fulfill their own metabolic needs and because of the inherent toxicity of free iron, animals use specific proteins, such as hemoglobin and ferritin, to carry and store iron, so that its concentration as a free species is kept extremely low at all times. ${ }^{[316]}$ In turn, animal hosts must mobilize their immune system to inactivate siderophores secreted by infectious microorganisms. ${ }^{[317,318]}$

Several different chemical functionalities, such as hydroxamates and carboxylic acids, are known to serve the purpose of iron chelating moieties. Particularly, catechol (o-dihydroxyaryl) units are often found in natural siderophores because of the excellent affinity for Fe"'. Simple catecholic molecules, such as 2,3-dihydroxybenzoylglycin -the first natural catecholic siderophore to be isolated -, ${ }^{[319]}$ 2,3-dihydroxybenzoic acid and 3,4-dihydroxybenzoic acid have been identified as natural siderophores, in the latter case secreted by microorganisms with unusually high iron requirements. ${ }^{[320]}$ Most catecholic siderophores, though, feature rather complex multipodal structures, having two or three catechol-based pendant groups attached to a common scaffold for a more efficient coordination of the metal center. Regardless of chemical functionality, the strongest natural siderophore known to date is enterobactin, ${ }^{[321,316]}$ a tripodal catecholic compound secreted by many Gram negative bacteria, among which $E$. coli. The chemical structure of enterobactin comprises three L-serine residues linked head-totail into a trilactone platform, to which are attached, cage-like, three pendant $\mathrm{N}-2,3-$ dihydroxybenzoyl groups. The six catecholic oxygen atoms define a hexadentate binding site, and are optimally directed for a strong octahedral coordination of the metal center. Similar, well-known tris-catechol-based siderophores are bacillibactin, ${ }^{[322]}$ secreted by Bacillus antracis, $^{[323]}$ and vibriobactin, isolated from Vibrio cholerae. ${ }^{[324]}$ Once bound to Fe", siderophores are recognized by specific receptors located on the outer membrane, which allow them to re-enter the bacterial cell. In order to maximize iron uptake capacities, single bacteria species are able to fabricate different types of siderophores,-such as catechol-based and hydroxamate-based-, for which they have several non-specific and specific receptors. ${ }^{[313,314]}$ In a complementary fashion, there exist a few mixed-ligand natural siderophores, such as petrobactin, in which chelating moieties of different nature are present simultaneously. ${ }^{[315]}$ 


\subsection{Artificial siderophores.}

Artificial siderophores are synthetic chelating agents of Fe"l intended as therapeutic agents specifically used for the treatment of both acute and chronic iron poisoning. The latter arises, for example, as a side effect in ß-thalassemia patients subject to chronic blood transfusions, leading to iron overload, for which the human body has no expedient remedy. Desferal ${ }^{\circledR}$ (desferrioxamine B mesylate, a hydroxamate-based drug) was approved more than 30 years ago and is still the most widely used siderophore drug. Despite being a strong in vitro ligand for iron, Desferal ${ }^{\circledR}$ shows limited in vivo bioavailability, and is not effective when administered orally, needing a continuous parenteral infusion for optimal efficacy, often conducive to low patient compliance. ${ }^{[325]}$

In the quest for new siderophore drugs with better pharmacological profiles, siderophore scaffolds based on catechol and catechol isosteres have been tested. ${ }^{[326]}$ Among many potential candidates, several small catechol-like derivatives, including 2,3-dihydroxybenzoic acid ${ }^{[327]}$ were already tested in vivo in the mid 1970s, of which 2,3-dihydroxynaphthalene-6sulfonic acid showed potential therapeutic activity. ${ }^{[328]}$ Among recently approved drugs, Ferriprox ${ }^{\circledR}$ (Deferiprone, or 3-hydroxy-1,2-dimethylpyridin-4-one, ${ }^{[329]}$ a small heterocycle and catecholate isostere, may be taken orally and has shown promising results compared to Desferal $^{\circledR}{ }^{[330]}$

Catechol derivatives as synthetic siderophores have been researched extensively. ${ }^{[331]}$ Early studies by Raymond and co-workers focused on homopodal enterobactin mimics like 1,5,9$\mathrm{N}, \mathrm{N}^{\prime}, \mathrm{N}^{\prime \prime}$-tris(2,3-dihydroxybenzoyl)cyclotriazatridecane, ${ }^{[332]}$ and 1,3,5-Tris-(2,3-dihydroxybenzoylaminomethyl)benzene, ${ }^{[333]}$ as well as heteropodal mimics such as 3,4 -LICAMS ${ }^{[325]}$ that preserved a tris-catecholate cage, but in these cases linked to backbone caps less prone to hydrolysis. In 3,4-LICAMS, sulfonic acid groups were added to the catechol rings for enhanced solubility and stability toward oxidation. Unlike Desferal ${ }^{\circledR}$, but in a similar fashion to enterobactin, 3,4-LICAMS was able to kinetically mobilize iron from transferrin in vitro, and showed that a rational strategy may be used for the design of ligands with optimal metal chelating properties. ${ }^{[334,331]}$ Research has also focused on the synthesis of mixed-ligand siderophores, in which at least one chelating moiety is catecholic, for example mono-catechol bis-hydroxamate- ${ }^{[335]}$ and bis-catechol mono-hydroxamate ligands. ${ }^{[335,336]}$ Other catechol-based ligands, such as 2,3-dihydroxyterephtalamide, have been used to prepare pure- and mixedligand siderophores with a very high affinity for Fe"', while at the same time being stable to oxidation and presenting low acidity. ${ }^{[337]}$ The use of systematic substitution of two different ligands in these siderophores proved effective in the fine-tuning of these critical physicchemical properties.

Catechol isosteres such as substituted 3-hydroxypyridin-2-ones (3,2-HOPO), ${ }^{[337,335,326]}$ and catechol-hydroxamate isosteres like 1-hydroxypyridin-2-ones $(1,2-\mathrm{HOPO}),{ }^{[338]}$ have been thoroughly studied with regard to the improvement of their metal chelating properties. In particular, it has been shown that a proper cap size facilitates an optimal accommodation of the ligands around the metal center. ${ }^{[39]}$ Moreover, the existence of a cap forming a joint hexadentate ligand structure dramatically increases the effectiveness of the ligand itself, compared with the analogous isolated (bidentate) ligand. With regard to their pharmacological properties, they showed low toxicity in vivo and higher efficacy than desferrioxamine. An additional advantage of 3,2-HOPO moieties over true catecholic residues lies in their higher 
acidity, which readily allows deprotonation of the hydroxy group for Fe" coordination at physiological $\mathrm{pH}^{[340]}$

Because of the sensitivity of catechol toward oxidation in the presence of air, especially at basic $\mathrm{pH}$, and oxidative species, such as Fe ${ }^{\text {III }}$ itself, the stability of catechol-based siderophores has to be taken into account for practical applications, and may be tuned by the electronic properties of the ring pendant groups. ${ }^{[341]}$ Of importance is also the hydrophilic/lipophilic balance in the siderophore, which can be adjusted by incorporating lipophilic side chains in the molecular structure, reminiscent of the amphiphilic siderophores secreted by certain marine bacteria. ${ }^{[32]}$ In buffered aqueous environments, synthetic amphiphilic siderophores have been shown to self-assemble into micelles and vesicles, ${ }^{[343]}$ or spherical particles $(100-300 \mathrm{~nm}),{ }^{[342]}$ depending on the presence or absence of a sulfonic acid substituent on the catechol ring. Kikkeri et al. have recently reported on a novel family of siderophores that combine siderophore-like catechol moieties with carbohydrate units arranged in dendritic structures. The authors showed that $E$. coli was able to use these chelating agents for iron uptake and growth promotion via specific sugar receptors in the cell membrane. ${ }^{344}$

In addition to their special affinity for iron, natural siderophores are effective chelating agents for other metals. The ligand properties of enterobactin toward trivalent species (AI'", Ga"', Sc ${ }^{\mathrm{III}}$, $\left(n^{I I I}\right),{ }^{[345]}$ and the crystal structure of a enterobactin- $V^{I V}$ complex have been reported. ${ }^{[346]}$ Other natural hydroxamate-catechol siderophores such as pyoverdin and azoverdin have been shown to form stable coordination complexes with $\mathrm{Ga}^{\mathrm{II \prime}}, \mathrm{Al}^{\mathrm{III},},{ }^{[347,348]}$ and $\mathrm{UO}_{2}{ }^{2+}$. $^{[349]}$ These complexes are so stable that the presence of $\mathrm{Al}^{\text {III }}$ and $\mathrm{Ga}^{\text {III }}$ interfere with $\mathrm{Fe}^{\text {III }}$ absorption in biological media. ${ }^{[347]}$ Not surprisingly, synthetic catechol siderophore mimics also form stable complexes with trivalent species. ${ }^{[345]}$ More intriguing is the biological role siderophores may play for nitrogen-fixating bacteria (diazotrophs), which must take in both $\mathrm{Fe}^{\mathrm{III}}$ and $\mathrm{Mo}^{\mathrm{VI}}$ for their metabolic process, particularly nitrogen fixation. Siderophore-Mo ${ }^{\mathrm{VI}}$ complexes are tetradentate, with two catechol moieties coordinating the vertices of a distorted octahedron, while the other two are occupied by oxygen atoms. Although it is still unknown if siderophores partake in $\mathrm{Mo}^{\mathrm{VI}}$ scavenging, or whether specific Mo-siderophore receptors exist in the outer cell membrane, catecholic bipodal siderophores such as azotochelin, ${ }^{[350]}$ and monopodal aminochelin, ${ }^{[351]}$ also bind $\mathrm{Mo}^{\mathrm{VI}}$ in vitro, which has been shown to delay the in vivo iron uptake by these siderophores. ${ }^{[352]}$

In this broader role as powerful generic transition metal ligands, synthetic siderophores have been tested for in vivo radioisotope decontamination. For tetravalent ions such as $\mathrm{Th}^{\mathrm{IV}}$ and $\mathrm{Pu}^{\mathrm{IV}}$, tetrakis-catechol-based ligands were specifically designed for optimal octadentate coordination and solubility in physiologic media. ${ }^{[353,354,355]}$ These ligands showed better in vivo $\mathrm{Pu}$ scavenging capacity than both $\mathrm{CaNa}_{3}$ DPTA and desferrioxamine $\mathrm{B}$, particularly at low actinide concentrations; moreover, the ligand was able to remove. ${ }^{[238]} \mathrm{Pu}$ already deposited in the skeleton. A more recent, detailed review covering the use of siderophores for actinide sequestration, ${ }^{[356]}$ concluded that, in general, tetradentate and octadentate catechol-, dihydroxyterephthaloyl- as well as isostere hydroxypyridone-based ligands are more efficient than DPTA-based ligands -the only ones approved for medical use-, and more selective toward actinides, especially in the case of Np, for which DPTA is not effective. ${ }^{[357]}$ Because of the high selectivity of each different ligand, a cocktail of ligands was suggested as a suitable prescription for generic radioisotope decontamination. Miao and Quinn reported an 
interesting reverse strategy involving catechol-radionuclide coordination in preliminary in vivo tests for peptide-targeted radionuclide therapy for melanoma, where radiolabeled peptides ended up selectively bound to 5,6-dihydroxyindole-based eumelanin and associated receptors, which are over-expressed by melanoma tumor cells. ${ }^{[358]}$

Using boron instead of Fe"', catechol coordination has been further explored by Messersmith and co-workers for cancer therapy. A modular molecule comprising PEG covalently linked to dopamine and fluorescence-tagged biotin was synthesized, where the catechol moiety was able to bind to a boronic-acid-based anti-cancer drug at basic $\mathrm{pH}$, and reversibly release said drug in acidic conditions. The biotin moiety allowed the assemblage to sneak through cell the membrane and thus be uptaken by cells, whereas the PEG moiety allows the complexed drug to survive longer in the body prior to liver degradation. By taking advantage of the fact that both cancer cells and extracellular interstices in tumors present lower-than-normal (acidic) pH, this strategy was proven to increase the selectivity of the uptake and release of this anticancer drug by cancer cells, as compared to normal cells. ${ }^{[359]}$

\section{2. "Trojan horse" therapeutic agents.}

Sideromycins are naturally occurring $\mathrm{Fe}^{\mathrm{III}}$-siderophores secreted by several fungi and bacteria species that are covalently linked to an antibiotic moiety. ${ }^{[360]}$ Using a Trojan horse or active transport strategy, sideromycins are channeled through the specific iron-uptake paths of competitor micro-organisms, which would otherwise be able to block the free antibiotic. Although most sideromycins are hydroxamate-based, a few natural catechol-based are known. For example, among microcins, gene-encoded antibacterial peptides with potent antibacterial activity against closely related species, ${ }^{[361]}$ - type E492 produced by Klebsiella pneumoniae was shown to consist of an enterobactin-like tris- $N$-(2,3-dihydroxybenzoyl)-L-serine end-cap, linked to a natural antibiotic oligopeptide via a glucose unit, ${ }^{[362,363,364]}$ whereas other microcins are suspected of requiring catecholate siderophore receptors for antibacterial activity. ${ }^{[365]}$

Synthetic siderophore-drug conjugates using a Trojan horse strategy have been widely researched because of their promising therapeutic value. ${ }^{[366,367,368,315369,370]}$ Since Fe ${ }^{\text {III }}$ uptake is essential to infectious bacteria, such synthetic conjugates may be used to target bacteria, like Pseudomonas aeruginosa, that are notorious for their natural and growing resistance to most known antibiotics. An early study on a catechol-cephalosporin drug already showed enhanced antipseudomonal activity of this conjugate with regard to the unmodified ß-lactam antibiotic. ${ }^{371}$ This effectiveness was lost on mutant strains in which the membrane iron-transport system had been suppressed, pointing unequivocally to a siderophore-dependent transport of the antibiotic inside the bacterial cell. ${ }^{[366]}$ Since cephalosporin and penicillin antibiotics are generally cheap and commercially available, their covalent conjugation to siderophores to obtain potent drugs is advantageous. ${ }^{[372]}$

In order to minimize resistance by receptor-deficient mutants, mixed-ligand siderophore-drug conjugates intended to target multiple receptors have been tested, for example carbacephalosporins bearing bis-catechol-mono-hydroxamate hybrid siderophores. ${ }^{[373,374,375,376]}$ It was suggested that selected mutant strains showing resistance would be, at the same time, severely iron-deprived for lack of proper iron-uptake routes, which would make them 
intrinsically less virulent. Moreover, despite the inability to deliver the antibiotic moiety, the presence of the siderophore-antibiotic conjugate in the surroundings of the infection would further contribute to the scarcity of available iron, still essential for bacterial growth. ${ }^{[373]}$

In addition to catechol-cephalosporin conjugates, ${ }^{[371,373,377]}$ which still represent one of the most actively researched siderophore-drug families, ${ }^{[368]}$ isostere 3-hydroxypyridin-4-one-based conjugates have been found to present excellent antibiotic efficacy against Staphylococcus aureus, ${ }^{[378]}$ and promising in vivo results for multi-drug resistant Acinetobacter baumannii strains. ${ }^{[379]}$ Other than ß-lactams, different kinds of antibiotics have been successfully conjugated to catechol moieties to render active antibacterial conjugates, such as quinolones. ${ }^{[380]}$ Further investigation has focused on improving the pharmacological properties of catechol-based drugs by esterification of the hydroxyl groups. ${ }^{[381,382,383,384]}$ It is claimed that the esters are cleaved under physiological conditions, which would explain the high activity shown by these "pro-drugs".

Apart from antibiotics, other conjugate partners for siderophores have been studied, such as porphyrins for photodynamic therapy. ${ }^{[385]}$ Finally, it should be mentioned that substituted catechols and catechol isosteres have been found to inhibit specific enzymes, such as lipooxygenases, ${ }^{[386]}$ and deoxyhypusylhydroxylases. ${ }^{[387,388]}$ In both cases, the chelation of the iron atom at the active site of the enzyme was assumed to be a key step in the inhibition mechanism.

\subsection{MRI contrast agents.}

Gadolinium complexes, among which novel ligands bearing catecholic and catechol-isostere units, have been thoroughly studied and recently reviewed, ${ }^{[389,390]}$ because of their potential in the on-going quest for highly effective contrast agents for magnetic resonance imaging (MRI). ${ }^{[391]}$ Homopodal ligands bearing three 3-hydroxypyridin-2-one $(3,2-\mathrm{HOPO})$ units, $^{[392]}$ and heteropodal variants of the same, where other catecholic chelators such as 2,3dihydroxyterephthalamide (TAM) substitute in for one of the 3,2-HOPO units, ${ }^{[393]}$ showed relaxivity values that doubled those of clinically employed polyaminocarboxylate complexes (Ca. $5 \mathrm{mM}^{-1} \cdot \mathrm{s}^{-1}$ ). The affinity of the ligand for $\mathrm{Gd}^{\prime \prime \prime}$ was found suitable to avoid transmetalation by physiological $\mathrm{Ca}$ "l leading to significant liberation of the in vivo-toxic, free lanthanide ion. These high relaxivities were explained by the fast exchange of water molecules between the 8and 9-coordinated species in equilibrium, in which water molecules enter and leave the two or three remaining sites that, respectively, complete the coordination sphere of the $\mathrm{Gd}^{\prime \prime \prime}$ atom. A screening of heteropodal variants of the mixed 3,2-HOPO/TAM ligands proved that it is possible to tune the overall basicity of the ligand in order to maximize the stability of the Gd"I complex at physiological $\mathrm{pH}^{[394,395]}$ An additional advantage offered by the inclusion of a TAM group was the possibility to functionalize the 4 position with PEG chains of varying length, which conferred varying degrees of hydrophillicity to the complex that correlated with a selective final in vivo distribution of the molecule, either to the liver or the blood pool. ${ }^{[396]}$ More recent attempts to increase the molecular relaxivity value of the Gd"I complex focused on the covalent binding of the optimized ligand to bulky supramolecular structures of high molecular weight and low tumbling rates. In one study, estearamide- and branched polylysinebased dendrimers were functionalized with up to 6 tripodal HOPO-based ligands. The best relaxivity values where obtained with the estearamide-based dendrimer $\left(38 \mathrm{mM}^{-1} \cdot \mathrm{s}^{-1}\right)$. These 
macromolecules offered an attractive alternative to other tested probes in view of their low in vitro cytotoxicity and favorable degradation profiles, as shown by the low toxicity values obtained after $72 \mathrm{~h}$ in in vitro biological conditions and up to milimolar concentrations of the dendrimer-based contrast agent. ${ }^{[397]}$ In another, biomimetic approach, capsids of the bacteriophage virus MS2 were emptied of RNA strands and both the exterior and interiorfacing lysine residues and exterior-facing tyrosine residues were functionalized with groups bearing terminal aldehyde groups, which in turn were reacted with a properly activated tripodal HOPO-based complex. The final functionalized capsids, bearing each up to 90 HOPO anchors for $\mathrm{Gd}^{\prime \prime \prime}$ complexation, showed very high relaxivity values $\left(30-42 \mathrm{mM}^{-1} \cdot \mathrm{s}^{-1}\right.$ per Gd"' unit) while at the same time retaining the necessary fast water-exchange capabilities. In the case of the interior-functionalized capsid ( $42 \mathrm{mM}-1 \cdot \mathrm{s}-1$ per Gd"I' unit), the latter took place thanks to the porous shell structure of the capsid. This was found particularly convenient, since the capsid may carry its payload (HOPO- Gd'l') concealed in the interior, while at the same time showing a clean outer surface available for biorecognition. ${ }^{[398]}$

Using a different supramolecular approach, Parac-Vogt and co-workers prepared a ditopic chelating ligand bearing both catechol and carboxylic acids groups that was shown to specifically bind trivalent lanthanide ions through the carboxylic acid moieties, and $\mathrm{Ti}^{\mathrm{iV}}$ via catechol groups. The resulting Gd-“metallostar” showed magnetic relaxivities almost an order of magnitude higher than a currently commercial MRI contrast agent. ${ }^{399}$

\subsection{Luminescent probes.}

Lanthanide complexes with ligands carrying catechol-isostere HOPO-based chelating units have been reported for their use as efficient luminescent probes in highly-sensitive fluorescence immunoassays. ${ }^{[400]}$ As opposed to traditional dissociation-enhanced assays (DELFIA), in which the photosensitizing species is added at the end of the assay, the purpose of these studies lies in finding efficient photosensitizers capable, at the same time, to chelate the metal ion in a strong and stable fashion in very dilute aqueous media and during long assay times. This way, an inherently highly fluorescent lanthanide-photosensitizer complex would be added at the beginning of the assay, and the fluorescent signal would be followed throughout, ideally without being impaired by the solvent. The first examples of HOPO-sensitized lanthanides were tetrakis-1,2-HOPO complexes of $\mathrm{Eu}^{\mathrm{III}}$ and $\mathrm{Tb}^{\mathrm{II \prime}}$, for which either poor, or efficient photosensitizing effects in solid-state samples were reported, respectively. ${ }^{[401]}$ Solution luminescence measurements were carried out on a dimeric Eu'l' complex of a bipodal 1,2-HOPO-based ligand, which was found to exhibit excellent stability in aqueous media and almost pure red fluorescence with a high quantum yield and long lifetime $(>700 \mu \mathrm{s})$. This was attributed, in part, to the fact that the metal center is well shielded from water molecules, thus dramatically decreasing the rate of solvent-induced fluorescent quenching. ${ }^{[402]}$ In contrast with 1,2-HOPO, ${ }^{[402,403]}$ similar 3,2-HOPO-based ligands did not sensitize Eu'", but were able to do so for Ho"I and Pr'", in this case in the NIR range, thus accounting for the few examples known of NIR emission by these lanthanide ions. ${ }^{[404]}$ Similar complexes showing exceptionally high quantum yield and very long lifetime luminescence in the NIR region and excellent stability in solution were prepared with bi- and tetrapodal 1-methyl-3,2-HOPO-based ligands with $\mathrm{Yb}^{\mathrm{II \prime}}$ and $\mathrm{Nd}^{\mathrm{II}}$, ${ }^{[405]}$ making them excellent candidates for bioassays, in which, because of the large separation between excitation and emission wavelengths, NIR imaging/detection should provide better resolution of the fluorescent signal. 


\subsection{Heavy metal scavenging.}

Heavy metal accumulation in the environment affects crop health and safety, ${ }^{[406]}$ as well as the health of soil micro-organisms. ${ }^{[407]}$ Although the natural propensity of plants to take up metals is detrimental to human health when intended as food sources, they offer, on the other hand, a tool for phytoremediation of contaminated soils. In order to enhance heavy metal mobilization and facilitate the uptake by the plant, chelating agents may be added to the soil. ${ }^{[408]}$ Among them, naturally occurring humic acids, made up complex, alkaline-soluble macromolecules bearing polyphenolic, catechol and quinoid moieties, have been studied as soil additives capable of heavy metal chelation and a subsequent increase in mobility and bioavailability of these pollutants to suitable, metal-hyperaccumulating plant species. ${ }^{[409]}$ Alternatively, several rhizobacteria known to be tolerant to heavy metals in the soil, ${ }^{[410]}$ can be inoculated, improving the uptake by so-called bioaugmentation. ${ }^{[411]}$ Particularly, the addition of free and encapsulated cultures of siderophore-secreting bacteria, like pyoverdin-producing Pseudomonas aeruginosa, in maize crop soils has been shown to increase the concentration of $\mathrm{Pb}^{\mathrm{II}}$ and $\mathrm{Cr}^{\prime \prime \prime}$ by 4 and 5-fold in crop shoots, as well as a positive heavy metal transport from the roots to the shoots. Tests with mutant siderophore-deficient strains would confirm the positive role of siderophores in the overall process. ${ }^{[412]}$

Certain fungi are known to secrete and accumulate insoluble, 5,6-dihydroxyindole-based melanins on the outer cell membrane. Melanized fungi show increased virulence and resistance to microbial attack as well as enhanced survival under environmental stress, particularly in heavy-metal contaminated soils, where pigmented strains have a survival advantage. ${ }^{[271,413,272,414]}$ For instance, Armillaria rhizomorphs has been shown to concentrate heavy metals 50-100-fold with regard to the soil content, ${ }^{[415]}$ whereas lichens have been found to inhabit uranium-polluted soils, where they use cell-wall melanin as a sequestrating agent. ${ }^{[46]}$ In this regard, the combined use of melanized fungi and certain clays has shown potential for the removal of radionuclides like ${ }^{90} \mathrm{Sr}$ and ${ }^{137} \mathrm{Cs} .{ }^{[417]}$

Synthetic catechol-based stationary supports have been explored for the scavenging of heavy metals from aqueous samples. The sequestering capacity of these substrates was first tested on Tiron ${ }^{\circledR}$-functionalized cellulose ${ }^{[418]}$ and polystyrene, ${ }^{[419]}$ as well as 3,4-dihydroxybenzylsubstituted poly(styrene-divinylbenzene, PS-DVB) ${ }^{[420]}$ which showed affinity toward Fe ${ }^{\text {III }}$ and $\mathrm{Hg}^{\prime \prime}$, respectively. As far as ion exchange resins are concerned, true selectivity for Fe ${ }^{\text {III }}$ in aqueous environments with regard to other trivalent ions -such as $\mathrm{Al}^{\text {III }}$ and $\mathrm{Cr}^{\text {III- }}$ - and divalent species, was achieved by Fish and co-workers by applying a biomimetic approach, by which bipodal and tripodal, siderophore-like catechol-based pendant groups were used to functionalize a PS-DVB resin. ${ }^{[421,422,423]}$ Changes in the scaffold to which the catechol groups were attached shifted the selectivity to divalent cations $-\mathrm{Ni}^{\prime \prime}, \mathrm{Zn}$ ", $\mathrm{Mn}$ ", $\mathrm{Cu}^{\prime \prime}$ - favoring squareplanar coordination. ${ }^{[424]}$ A similar strategy was applied to non-aqueous media for the decontamination of oil shales and related pyrolysis products, ${ }^{[425,426,427]}$ where a suitably modified PS-DVB resin showed chelating capacity toward arsenic and organoarsonic acids. The resin could be regenerated with quantitative recovery of As in the first $10 \mathrm{~min}$.

In the past years, catechol-derivatized porous substrates have been studied regarding their use in analytical methodologies involving the pre-concentration of heavy metals present in trace amounts in waste waters and commercial drugs. In this case, emphasis was put not on the 
selectivity of catechol residues toward a specific species, but on the general chelating properties of the catechol moiety, ${ }^{[428]}$ and hence on its potential overall sequestering capacity of a wide variety of common metal pollutants. The polymeric support was thus used as an accumulator for the analytes, which were later quantitatively released in a much highly concentrated flow, coupled downstream to an analytical technique of appropriate sensitivity. So far, Amberlite XAD-2, ${ }^{[429,430,431]}$ XAD-4, ${ }^{[432,429]}$ XAD-16, ${ }^{[433,434,435]}$ XAD-1180, ${ }^{[436]}$ polyurethane foam, ${ }^{[437]}$ polyacetylene, ${ }^{[438]}$ silica, ${ }^{[439]}$ and cellulose, ${ }^{[440]}$ have been covalently functionalized with catechol moieties, and successfully used to quantify trace amounts of $\mathrm{Cu}^{\prime \prime}, \mathrm{Ni}^{\prime \prime}, \mathrm{Pb}^{\prime \prime}, \mathrm{Cd}^{\prime \prime}$, $\mathrm{Zn}^{\prime \prime}, \mathrm{Co}{ }^{\prime \prime},{ }^{[441,429-408,437,438]} \mathrm{Mn}^{\prime \prime},{ }^{[432,433,430,435]} \mathrm{UO}_{2}{ }^{2+},{ }^{[432,433,442,443]}$ and $\mathrm{Th}^{\mathrm{IV}} .{ }^{[442]}$ In most cases, supports were easily regenerated and re-used multiple times. In a recent study, 3,4dihydroxybenxene-functionalized and pre-cross-linked chitosan was tested against 60 prospective trace elements, ${ }^{[443]}$ and later used for the continuous analysis of $\mathrm{Ag}^{\prime}, \mathrm{Bi}^{\mathrm{II \prime}}, \mathrm{Cu}^{\prime \prime}, \mathrm{Ga} \mathrm{G}^{\prime \prime \prime}$, $\mathrm{In}^{\mathrm{III}}, \mathrm{Mo}^{\mathrm{VI}}, \mathrm{V}, \mathrm{Ni}^{\mathrm{II}}{ }^{[444]}$ and $\mathrm{U}^{\mathrm{IV}}$, proving particularly useful for the quantitative determination of the latter. ${ }^{[443]}$

An alternative to the modification of commercially available microporous substrates was the fabrication of copolymers of 3,4-dimethoxystyrene (DMS) with divinylbenzene (DVB), with subsequent deprotection of the hydroxyl groups to yield a cross-linked vinylcatechol-DVB copolymer. In this case, the monomer ratio was found to be a compromising key factor, affecting both the degree of porosity and the chelating capacity of the resin. ${ }^{[45]} \mathrm{A}$ similar approach was used in the synthesis of a terpolymeric resin of vinylbenzene, divinylbenzene and $\mathrm{N}$-substituted maleimide with a 3,4'-dihydroxyphenyl pendant group. In this case, the dihydroxyphenyl moiety was reacted with $\mathrm{Ti}(\mathrm{PrO})_{4}$ to yield novel porous catalytic resins in which Ti coordinated with the hydroxy groups. These resins were found to exhibit very good catalytic activity toward model transesterification and epoxidation reactions. ${ }^{[46]}$ 


\section{Materials for chemo-/biosensing.}

Several quinones have been identified as co-factors of so-called quinoproteins, which include certain dehydrogenase and oxidase enzymes. In general, quinoid co-factors are involved in enzymatic oxidation processes in which o-quinone moieties oxidize specific substrates -usually alcohols and amines-, after which the corresponding reduced species is regenerated by an assisting electron acceptor. ${ }^{[447]}$ Among o-quinoid co-factors, free pyrroloquinoline quinone $(\mathrm{PQQ}),{ }^{[448]}$ and enzyme-bound lysine tyrosylquinone (LTQ) ${ }^{[449]}$ likely the most extensively researched to date, are known to oxidize alcohols and primary amines, respectively, to the corresponding aldehydes. ${ }^{[48,450]}$ Particularly in the case of PQQ, the reduced species is assumed to be the catecholic dihydroxy compound, ${ }^{[451]}$ which exemplifies the reversible redox role of the $o$-quinone/catechol pair in living organisms. In this regard, it has been suggested that PQQ may even qualify as a vitamin, because of its crucial role as a redox co-factor in the oxidative degradation of lysine observed in mice, ${ }^{[452]}$ although this consideration still remains controversial. ${ }^{[453,454]}$ From a biomimetic point of view, quinoid co-factors can thus be seen as paradigms of $o$-quinoid species thay may be reversibly converted into catecholic species by means of applied external stimuli, be it an electric potential (biosensors, biofuel cells, electrochromic materials and related devices), thermal energy (valence tautomerism) or electromagnetic radiation (dye-sensitized solar cells).

\subsection{NADH biosensing electrodes.}

The NADH/NAD redox pair is an essential coenzyme found in hundreds of dehydrogenase enzymes, which has led in the past years to a considerable interest in the design of different kinds of enzyme-based amperometric sensors. In solution, NADH is electrochemically oxidized at high overpotentials in an irreversible two step process involving an unstable radical intermediate species. ${ }^{[455]}$ Because of this, electrodes based on the direct electrooxidation of NADH are subject to fast fouling, limiting in principle the development useful NAD/NADHbased electrochemical devices. Efforts to overcome this hurdle started with an early study by Blaedel and Jenkins, who showed that pre-conditioned glassy carbon electrodes (GCE) afforded a significant decrease in the oxidation overpotential of NADH. The prior treatment of the electrode surface consisted of a series of repeated redox cycles, which afforded a chemically modified surface with different carbon oxide groups, such as carboxy, carbonyl, and quinoid structures. The authors hypothesized that these groups might be responsible for the observed decrease in the oxidation barrier of $\mathrm{NADH} .{ }^{[456]}$

Drawing on these results, Kuwana and co-workers suggested that a controlled chemical modification of electrodes might afford a satisfactory strategy for the selective oxidation of $\mathrm{NADH}{ }^{[457]}$ According to this approach, an appropriate mediator species is physically or chemically bound to the electrode. When oxidized electrochemically, the mediator chemically oxidizes the final species of interest (NADH). Although many mediator species can be used for the oxidation of $\mathrm{NADH}, o$-quinones have been shown to be especially apt for this purpose, because in addition to appropriate thermodynamic driving forces, they generally afford high reaction turnovers - particularly critical in the case of electrode-deposited monolayers of the mediator-. ${ }^{[458,459]}$ Indeed, the kinetics of $o$-quinoid oxidation of $\mathrm{NADH}$ in aqueous media were 
explained after a series of detailed investigations by Miller et al., which revealed that quinones in general -and particularly o-quinones-, oxidize NADH by direct hydride transfer. This mechanism presents a lower energy barrier, compared with one-electron transfer processes, such as those afforded by the ferrrocenium ion, and is thus kinetically favored. ${ }^{[458]}$

Different mechanisms to attach redox mediators to electrode surfaces have been tried so far, among which direct adsorption ${ }^{[460,461]}$ or covalent binding ${ }^{[462,463,464]}$ of monolayers of the redox mediator, in situ electropolymerization, ${ }^{[465,466]}$ immobilization in a pre-cast lipidic film, ${ }^{[467,468]}$ direct mix in a carbon paste, ${ }^{[469]}$ and deposition of a polymer bearing redox moieties. ${ }^{[470]}$ Examples of catecholic compounds explored as suitable redox shuttles include pyrocatechol, $^{[471,472,473,474]}$ 3,4-dihydroxybenzaldehyde, ${ }^{[475]}$ 3,4-dihydroxybenzylamine, ${ }^{[476,477]}$

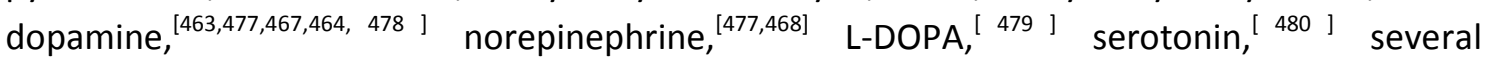
arylalkylcatechols, ${ }^{[460,461]}$ chlorogenic acid, ${ }^{[481]}$ caffeic acid, ${ }^{[482]}$ nordihydroguaiaretic acid, ${ }^{[483]}$ pyrocatechol violet, ${ }^{[484]}$ lignosulfonic acid, ${ }^{[485]}$ and catecholic flavonoids, such as rutin ${ }^{[486]}$ and quercetin. ${ }^{[464,487]}$ A recent study by Bartlett and co-workers on the reversible catalytic oxidation of NADH systematically screened 13 different dihydroxybenzene derivatives covalently linked to GCEs by means of two different spacers, showing how the substitution pattern of the aryl ring affects both the redox potential and the surface coverage of the electrode. ${ }^{[488]}$ As far as the final analyte is concerned, NADH has been studied preferentially, although other redox active species have been successfully detected with catechol-based electrochemical sensors, such as ascorbic acid, ${ }^{[486,468]}$ phenylalanine, ${ }^{[469]}$ dopamine ${ }^{[464,477]}$ and captopril. ${ }^{489]}$ Finally, although much work has been done on the modification of glassy carbon, graphite and metal electrodes with catecholic species, more recent work has explored the use of carbon nanotubes for the encapsulation of such materials for the detection of dopamine, ${ }^{[487]}$ redoxsensitive drugs, ${ }^{[489]}$ the electrocatalyzed oxidation of hydrazine, ${ }^{[474]}$ and the catalyzed oxidation of $\mathrm{NADH}{ }^{[490,480]}$ A recent report by Gorski et al. showed that carbon nanotubes may be themselves activated for catalytic NADH oxidation by simply boiling them in water. Not only was this treatment mild and non-destructive, but the catalytic effect was much higher than that achieved by surface treatment of other carbon allotropes such as glassy carbon, graphite powder, and edge-plane pyrolytic graphite. As in the original work by Blaedel and Jenkins on GC, o-quinoid structures generated in the course of surface treatment were invoked as responsible for the catalytic effect. ${ }^{[491]}$

Electropolymerization was chosen by Miller and co-workers to deposit PMMA polymer with pendant dopamine moieties on a GCE. The quinoid-rich film showed electrocatalytic activity toward the oxidation of NADH, which was highest at a monolayer degree of coverage, for which most dopamine units could be effectively oxidized to the corresponding dopaquinone species. In multilayer coatings, only the innermost layer -in direct contact with the electrodeprovided significant amounts of electroactive dopaquinone necessary for the oxidation of $\mathrm{NADH}$, while the outer polymer layers remained electrochemically inactive and acted as a barrier for the diffusion of NADH. The addition of electroactive species capable of mediating one-electron transfer processes, such as soluble ferrocene derivatives, were shown to enhance the catalytic activity of multilayer coatings, likely by mediating electron transfers between the inner and the outermost layers. ${ }^{[470,492,493]}$

Jaegfeldt and co-workers adsorbed two different arylvinylcatechols on graphite electrodes taking advantage of the affinity between polycyclic aromatic moieties and the graphite surface. The electrode coated with 4-[2-(2-Naphthyl)vinyl]catechol showed electrocatalytical 
activity towards NAD/NADH. ${ }^{[460]}$ When a more extended polycyclic aromatic system such as vinylpyrene was used as anchoring moiety, a detailed study of the catalysis kinetics toward NADH showed a progressive inactivation of the electrode, which was attributed to the formation of stable C-O-C covalent links between NADH and catechol moieties, arising from the coupling between radical species. ${ }^{[41]} \mathrm{A}$ similar NADH-induced inactivation process was also observed by Kuwana and coworkers on 3,4-dihydroxybenzylamine covalently bound to GCE. In contrast with $\mathrm{NADH}$, ascorbic acid (AA) did not lead to electrode inactivation after successive redox cycles. ${ }^{[476]}$

The electropolymerization of catechol and small catecholic derivatives has been investigated widely because of its affordability and convenient solubility in biological media, which allows the in situ preparation of films. ${ }^{[41]}$ Abruña and co-workers studied the catalytic activity of electropolymerized films of 3,4-DHB on GCEs, ${ }^{[475]}$ as well as other formyl-substituted $o$ - and $p$ dihydroxyaryl compounds. ${ }^{[465]}$ Films were prepared by potential cyclization and deposition at a constant potential, the latter method affording the most stable ones. Catalytic activity toward NADH was strong enough to detect this species in the micromolar range. ${ }^{[494]}$ In a later study, an alcohol sensor based on a NADH-dependent alcohol dehydrogenase enzyme was developed. Undesired interference of ascorbic acid in the measurements was eliminated by adding ascorbate oxidase, either in solution or immobilized on a nylon mesh. ${ }^{[495]}$ Using an alternative linking strategy for 3,4-DHB, the Schiff base of this compound and 4-aminopyridine was deposited on a Pt electrode, affording an electrocatalytic SAM for the oxidation of NADH. ${ }^{[462]}$ By means of a similar strategy, Chen and co-workers used a cysteinamine-glutaraldehyde conjugate monolayer on a Au electrode to immobilize dopamine for the same purpose. ${ }^{[463]}$ Catechol and several catechol derivatives have been electropolymerized on GCE, yielding polymeric films that retain redox properties, as opposed to passive films obtained on metal electrodes. When this process was carried out in the presence of 1,3-diamines reactive toward $o$-quinoid species, the redox activity of these films was observed to change significantly. ${ }^{[471]}$ Advantage was taken of the reactivity of o-quinoid species, in this case toward different thiolated aminoacids, by Compton and co-workers to devise a protocol for the selective electrochemical detection of cysteine in the presence of with little interference from cystine, homocysteine and glutathione. ${ }^{[496]}$

Heterocyclic 0 -quinones with structures reminiscent of PQQ have been explored by different groups, ${ }^{[466,497,498]}$ as electrocatalytic species for NADH and other species. In an early study, Abruña and co-workers showed that mixed-ligand transition metal complexes with 2,2'bipyridine (bpy), 1,10-phenanthroline (phen) and 1,10-phenanthroline-5,6-quinone (phendione), such as $\left[\mathrm{Ru}(\mathrm{bpy})_{2} \text { (phendione) }\right]^{2+}$, catalyze the oxidation of NADH in solution. The strongest effect was observed for a thin film of $\left[\mathrm{Os}(\text { phen })_{2}(\text { phendione })\right]^{2+}$ on a pyrolytic graphite electrode. ${ }^{466]}$ Further investigations with similar complexes, such as [Fe(phendione $\left.)_{3}\right]^{2+},{ }^{[499]}$ and $\left[\operatorname{Re}(\text { phen-dione })_{3}\right]^{2+},{ }^{[500]}$ afforded electrodeposited thin films on GCEs that showed a dramatic catalytic effect, together with good stability, with no interference of ascorbate. An ethanol biosensor was fabricated by immobilizing an alcohol dehydrogenase enzyme on a nylon mesh and using the iron-phenanthrolinedione complex deposited on the chemically modified electrode as redox mediator for the regeneration of the NAD cofactor. ${ }^{[499,500]}$ 


\subsection{Other biosensing electrodes.}

Valdés and co-workers studied the catalytical activity of dopamine, noepinephrine and 3,4dihydroxybenzylamine in solution toward a FAD/FADH2-dependent glucose oxidase. In view of the catalytic effect, it was suggested that the electrochemical degradation of catecholamines to yield indolic structures by intramolecular reaction might be diminished by rapid reaction of the oxidized catecholamines with the FADH2 cofactor. The authors reported the development of a method to detect catecholamines by adapting an electrochemical sensor with immobilized GOx on graphyte electrodes to a HPLC flow-cell. ${ }^{[477]}$ In another approach to the detection of dopamine, Rivas et al reported the generation of a stable melanin-like film of electropolymerized L-DOPA on a glassy carbon electrode in such conditions that the film surface was rendered negatively charged, which allowed the exclusion of anionic species as potentially interfering with the analysis of species of interest, such as catechol and several catecholamines. Ascorbic acid was used in solution to amplify the oxidation signal in the detection of dopamine down to $5 \mathrm{nM} \cdot{ }^{[479]}$ Ensafi and co-workers prepared a modified carbon paste electrode by mixing graphite, carbon nanotubes and a catechol derivative acting as a redox mediator for the determination of antihypertension drug captopril in pharmaceutical preparations and urine, taking advantage of the redox properties of the terminal -SH group in captopril. Differential pulse voltammetry using such electrode showed improved sensitivity toward established analytical methods, as well as a high selectivity, even in real (urine) samples. ${ }^{[489]}$

Kim and co-workers fabricated a lactate dehydrogenase screen-printed biosensor in which the printing ink was formulated with graphite, $\mathrm{NADH}$, L-lactate, a hydroxymethylcellulose-ethylene glycol polymeric matrix and 3,4-DHB as electron shuttle. The detection limit thus achieved (50 $\mathrm{U} / \mathrm{L}$ ) closely approached the clinically lower range of concentration $(40 \mathrm{U} / \mathrm{L}){ }^{[501]}$ The same redox mediator was chosen by Weiss and co-workers to design the first reagent-less dehydrogenase-based biosensor for monitoring phenylalanine in human urine. In this study, 3,4-DHB was directly mixed with the carbon paste, instead of being used to modify the surface of the electrode with an electroactive film. Because of this, this electrode could be easily regenerated by simple polishing. ${ }^{[469]}$

A sophisticated approach was devised by Scheller and co-workers in order to develop a highsensitivity biosensor for alkaline phosphatase, in which this enzyme converted phenyl phosphate into phenol, which was subsequently transformed into catechol by means of a tyrosinase. Catechol then entered an amplification sensing loop comprising several mutuallycoupled redox cycles; among these one in which this species was reversibly oxidized to obenzoquinone, and rapidly regenerated by a PQQ-dependent glucose-oxidase. This biosensor was successfully applied to the fast detection of traces of a pesticide by means of a competitive ELISA assay, showing enough sensitivity to comply with the limits established by the European legislation. ${ }^{[502]}$ More recently, Lorenzo and co-workers fabricated a DNA biosensor based on $\left[\mathrm{Os}(\mathrm{bpy})_{2}(\text { phendione })\right]^{2+}$ and $\left[\mathrm{Os}(\text { phen })_{2}(\text { phendione })\right]^{2+}$. A single strand of DNA was thiol-immobilized on a gold electrode and then allowed to hybridize with a second strand. Finally, the phendione complexes were electrodeposited on the Au electrode and differential pulse voltammetry recorded. The authors observed a significant change in peak electric current only for DNA strands hybridized with complementary sequences. ${ }^{[498]}$ 


\subsection{Enzymatic biofuel cells.}

o-Quinoid Pyrroloquinoline quinone ( $\mathrm{PQQ}$ ) has been extensively investigated as a redox mediator for enzyme-based electrodes and biofuel cells. In an early study, Katz and co-workers prepared covalently immobilized $\mathrm{PQQ}$ on $\mathrm{Au}$ and $\mathrm{Pt}$ electrodes and demonstrated the reversibility and stability of this modified electrode over a wide $\mathrm{pH}$ range. ${ }^{[503]}$ The catalytic activity of the electrode-bound co-factor towards the oxidation of NADH was significantly enhanced by the addition of $\mathrm{Ca}^{2+}$, reminiscent of the enzymatic in vivo oxidation mechanism. ${ }^{\text {[504] }}$ Later, Willner and co-workers fabricated a functioning enzyme-less biofuell cell by using a PQQ-modified electrode as anode. ${ }^{[505]}$ Simultaneously, this research group made a significant brakethrough in the development of enzymatic biofuel cells by means of a "reconstitution strategy": The cofactor of a redox protein was carefully extracted, covalently linked to an electrode in a self-assembled monolayer -either directly, or through a suitable redox mediator, such as PQQ-, and finally joined back with the apo-enzyme. This approach ensured a good electrical contact between the redox protein and the electrode, while at the same time preserving the redox activity of the protected, still-functional cofactor. ${ }^{[506]}$ In follow-up studies, PQQ was successfully used as redox mediator, electrically connecting Au electrodes with FAD and NADH cofactors for glucose oxidase $(\mathrm{GOx})^{[507,508]}$ and lactate dehydrogenase (LDH) -based electrodes, ${ }^{[509]}$ respectively. After the optimization of the Au - PQQ - cofactor connection, ${ }^{[510]}$ the turnover rate of the associated glucose oxidase reached that of this enzyme with its native, in vivo $\mathrm{O}_{2}$ electron acceptor. ${ }^{[511]}$

More recent research of the Willner group focused on the functionalization of $\mathrm{Au}$ nanoparticles ${ }^{[512]}$ acting as last electrical relays in modified electrodes. In one study, PQQ itself was chosen as redox cofactor -instead of mediator-, for the fabrication of a glucose dehydrogenase-based electrode that showed high enzymatic turnover rates and efficient electric contact between the protein and the electrode, albeit also a considerable oxidation overpotential. ${ }^{[513]}$ Following another line of research, $\mathrm{Fe}_{3} \mathrm{O}_{4}$ NPs were linked to PQQ relays and $\mathrm{NADH}$ cofactors. In this case, the modified NPs were not covalently attached to the $\mathrm{Au}$ electrodes, but were instead suspended freely in the medium. It was shown that the catalytic activity of the enzyme, which as usual relied on the electrochemical regeneration of the cofactor, could be switched off by dragging the NPs away from the electrode by means of a magnetic field, and subsequently turned on when the field was reversed and the NPs approached and eventually contacted the anode. ${ }^{[514,515]}$ Finally, this research group demostrated that a small enhancement in the catalytic activity of NAD/LHD-based biofuel cells using PQQ as mediator redox species was observed when a constant magnetic field was applied. The authors quantitatively explained this effect with a magnetohydrodynamic model. [516]

\subsection{Chemosensors.}

A few catechol-based molecules have been found useful in the selective sensing of certain "difficult" species, such as ammonium cation ${ }^{[517]}$ and biologically relevant Mo, $\mathrm{W}$, and $\mathrm{V}$ oxometalates. In this case, a catechol moiety was covalently linked to a luminescent $\mathrm{Re}$ coordination complex. ${ }^{[518]}$ Upon complexation of the oxometalates at low $\mathrm{pH}$, the catechol deprotonated and luminescence was quenched. This effect was not observed in the presence of other common transition metals that coordinate catechol efficiently, but do so at higher $\mathrm{pH}$ 
values, such as $\mathrm{Co}^{\prime \prime}, \mathrm{Ni}^{\prime \prime}, \mathrm{Cu}^{\prime \prime}, \mathrm{Zn}^{\prime \prime}$, and $\mathrm{Fe}^{\mathrm{III}} .^{[519]}$ Dioxometalate complexes with substituted catechols afforded as well receptors with strong affinity for glutaric and glutamic acids, ${ }^{[520]}$ and short-chain diammonium salts. ${ }^{[521]}$ In addition to metal cations and organic species, catechol derivatives have been used as sensor moieties for a few anions. Simple molecules like pyrocatechol itself have been shown to associate favorably to halides via hydrogen bond. The effect was strong enough to be competitive with the chloride-binding capacity of a reference amide compound, as detected by ${ }^{1} \mathrm{H}-\mathrm{NMR} \cdot{ }^{[522]}$ When catechol moieties were supported, siderophore-like, in a scaffold of appropriate geometry and flexibility, the affinity of the ligand toward $\mathrm{Cl}^{-}$outperformed that of pyrocatechol. ${ }^{[523]}$ Fluoride could be detected selectively with catechol-chromophore conjugates, ${ }^{[524,525,526]}$ in which case both hydrogen-bond and acid-base mechanisms were claimed to explain the binding effect. Catechol-based anion recognition and transport in biological media has also been researched. Two catechol moieties were linked to scaffolds of variable amphiphilicity, showing that the molecules are able to catalyze anion transport through liposome membranes. The selectivity observed for the optimized biscatecholic compound with regard to the anion transport followed the sequence $\mathrm{ClO}_{4}{ }^{-}>\mathrm{I}^{-}>$ $\mathrm{NO}_{3}{ }^{-}>\mathrm{Br}^{-}>\mathrm{Cl}^{-}$, which correlated to their relative hydration energies (Hofmeister effect), as seen for many natural anion transport mechanisms. Also, the bis-catecholic transporter seemed to associate on the membrane at high concentrations, making the anion transport more efficient. ${ }^{[527]}$

Finally, the synthesis and characterization of a new family of catechol derivatives designed to behave as fluorescent chemosensors for wide-range $\mathrm{pH}$ detection has been described. ${ }^{[528]}$ These compounds were prepared by covalently coupling a catechol unit with other aromatic rings, thus obtaining $\pi$-delocalized systems with both $\mathrm{pH}$-responsive groups and fluorescent behavior. In the case of a pyridine-catechol derivative, this leads to up to three different protonation states with distinct optical properties in organic media, as corroborated by density functional theory calculations. By applying dualwavelength detection techniques, this compound shows complementary "off-on-off" and "on-off-on" emission profiles upon pH variation, a behavior that can be exploited to perform acidity detection over a broad $\mathrm{pH}$ range. The same authors also reported a new synthetic route for the synthesis of a sterically protected azobisphenol, obtained upon reaction of two catechol units, which exhibits a threestage sensor capacity. The reversible deprotonation/protonation of the phenol groups has been shown to take place under basic and acidic conditions, respectively. Moreover, this compound also exhibits good reactivity with transition-metal ions such as cobalt. Accordingly, combination of both the acid-base character and the complexation ability have been used to create a chromophoric array of three states with significantly different colours (enhanced by the presence of the azo group), which can interconvert reversibly between them. ${ }^{529]}$ In addition to the studies on bulk solutions, these families of compounds have alwso been structured on surfaces for the development of a surface molecular sensor for the detection of acidity. Initially, lithographically controlled wetting deposition has been applied to nanostructure a new fluorescent compound with three protonation states featuring different optical properties on a glass substrate. Atomic force microscopy demonstrates the functionalization of the surface with ordered arrays of the sensor molecules. The fluorescence properties of the resulting nanopattern at different $\mathrm{pH}$ values have been investigated by confocal fluorescene microsopy, thus revealing the fast, sensitive, reversible response of the prepared nanosensor to gas flows of varying acidity. ${ }^{[530]}$ Arrays of fluorescent $\mathrm{pH}$-responsive multistate molecules on surfaces have been fabricated by means of other soft lithographic techniques, such as $\mu \mathrm{CP}$. Direct physisorption onto the desired surface can be attained in a 
very simple manner, without the need for tedious modification of the molecular systems neither of the supporting platforms. However, the arrays lack of the stability required to be used with liquids. This situation is solved by the use of covalent bonding though this requires a previous functionalization of both the molecule and the surface. Interestingly, their acid-base switching behavior remains once structured on a surface with small variations of their emission spectrum. In this way, the patterns generated have shown fluorescence sensing capabilities when they are exposed to atmospheres of different acidity over large $\mathrm{pH}$-windows. These results open the door to the development of novel molecular-based sensing capabilities thanks to their fast responses. ${ }^{[531]}$ 


\section{Switching materials}

\subsection{Electronically labile transition metal complexes.}

The term of non-innocent ligands directly related to their role and the influence on the final electronic distribution of certain transition metal complexes was first pointed out by Jørgensen. ${ }^{[532]}$ This concept can be used when both the metal and ligand-centred electronic frontier orbitals are similar in energies. Within such scenario, the final oxidation state of the complex and the internal electronic distribution may be modulated becoming a great subject of investigation both by theoretical, ${ }^{[533]}$ and experimental means. ${ }^{[534]}$ One of such families of non-innocent ligands is that of $o$-quinone ligands, which can be found in transition metal complexes in two different oxidation states, radical semiquinones (SQ) or dianionic catecholates $\left(\mathrm{Cat}^{2-}\right)$. There would exist a third oxidation state, a neutral quinone (Q), though it can hardly be found coordinated to metal ions due to the limiting binding ability of the quinone ligand. ${ }^{[535]}$ Thanks to this rich redox activity, in some complexes bearing electro-active metal ions it is possible to induce an intramolecular electron transfer (IET) between the catechol and the metal ion by means of different external stimuli, mainly temperature, though also by light or pressure. The interest for studying this family of complexes is considerable since they are unique model systems which provide insight into the basic factors affecting intramolecular electron transfer. For this reason, this family of complexes is of great relevance on the field of molecular electronics as well as on the study of complexes bearing noninnocent ligands that perform physiological functions on biological systems. Both approaches are revised next.

\subsubsection{Molecular Electronics}

Valence tautomeric (VT) metal complexes with at least two redox-active centres, the metal ion and a catechol-based ligand, are characterized by the existence of two electronic isomers (valence tautomers) with different charge distributions. ${ }^{[536]}$ The interconversion between the different electronic isomers is accomplished by a reversible intramolecular electron transfer involving the metal ion and the redox active ligand. For this to take place, the degree of covalency in the interaction between the metal and the ligand must be low whereas the matching between the energy of their frontier orbitals similar. ${ }^{[537]}$ The first description of the above mentioned charge distribution sensitivity was the cobalt bis(quinone) complex [Co'I'(3,5DTBCat)(3,5-DTBSQ)(bpy)] (X), ${ }^{[538]}$ where 3,5-DTBCat ${ }^{2-}$ and 3,5-DTBSQ refer, respectively, to the catecholate (DTBCat ${ }^{2-}$ ) and semiquinonate (DTBSQ) forms of 3,5-di-tert-butyl-o-quinone, and bpy is 2,2'-bipyridine. In solution, the equilibrium in Equation 1 can be induced by variations of temperature and monitored by magnetic measurements and spectroscopic techniques such as UV-VIS, NMR and/or EPR. Since then, several other examples of monomeric, dimeric and even polymeric VT materials with not only cobalt but a series of transition metal ions such as $\mathrm{Cu}, \mathrm{Rh}$ and $\mathrm{Ir}, \mathrm{Ni}$ and $\mathrm{Mn}$ have been reported most of them being compilated in different reviews, to which the reader is further referred. ${ }^{[539]}$ Suffice it to say an introduction to the origin and behavior of this interesting family of switchable materials with emphasis in some specific aspects, mainly description of the different external stimuli that can be used for the interconversion, redox characteristic of different catechol-based Ligands and influence of the macroscopic state and matrix environment on the VT equilibrium.

$$
\left[\mathrm{Co}^{\prime \prime \prime}(3,5-\mathrm{DTBCat})(3,5-\mathrm{DTBSO})(\mathrm{bpy})\right]\left(\mathbf{x}, / \mathrm{s} \text {-Co(III)) } \rightleftharpoons\left[\mathrm{Co}^{\prime \prime}(3,5-\mathrm{DTBSO})_{2}(\mathrm{bpy})\right](\mathbf{x}, h s-\mathrm{Co}(\mathrm{III}))\right.
$$


$\mathrm{VT}$ is an entropy-driven process due to the higher spin state degeneracy and the higher density of vibrational states of the $h s$-Co(II) form due its longer metal-ligand bond lengths. ${ }^{[540]}$ Thus thermal population of tautomeric states is dictated by the Gibbs free energy expression. At low temperatures, $\mathrm{T} \Delta \mathrm{S}$ is negligible compared to $\Delta \mathrm{H}$, and consequently if $\Delta \mathrm{H}>\mathrm{kT}$ only the $/ s$ - $\mathrm{Co}$ (III) state is populated. An increase of the temperature will increase the T $\Delta \mathrm{S}$ contribution, making it non negligible and favouring the population of the $h s$-Co(II) state, up to a critical temperature $T_{\mathrm{c}}$ where $\Delta \mathrm{G}=0$ and $\Delta \mathrm{H}=\mathrm{T} \Delta \mathrm{S}$. A further increase of the temperature will change the sign of $\Delta \mathrm{G}$, being the $h s$ - $\mathrm{Co}(\mathrm{II})$ the most populated state. It is important to emphasize that VT can be induced not only by temperature variations but also by irradiation, as pioneered by Hendrickson ${ }^{[541]}$ and Dei, ${ }^{[542]}$ both in solution and in the solid state. ${ }^{[543]}$ Examples of pressureinduced VT have also been reported although in less extent than thermally-driven or lightinduced transitions. In these experiments, the increase of the molecular size on passing from the low to the high-spin isomer due to the population of antibonding orbitals is used to favour the low-spin isomer after application of pressure as an external stimulus. ${ }^{[544]}$

In addition to the use os different external stimuli, another area of special interest has been the finding of new redox-active Ligands that also show VT phenomena. ${ }^{[545]} \mathrm{A}$ further step in this sense has been the study and characterization of catechol-based O,N,O-coordinated type ligands containing two phenolate donor groups such as ligand BQ-N-SQ ${ }^{[546]}$ These ligands, in addition to producing phenoxyl radicals in the presence of air, exhibit better chelating capabilities and good $\pi$-donor atoms that stabilize higher oxidation states. ${ }^{[547]}$ The synthesis and mechanical features for the obtaining of the ligand BQ-N-SQ was first described in 1975 by Girgis and Balch, ${ }^{[548]}$ treating the 3,5-di-tert-butyl-catechol with aqueous ammonia in the presence of a divalent metal ion under aerobic conditions. Since then, a considerable interest has increased in the scientific community for this family of complexes. Thanks to their rich redox activity, these ligands may exist at least in four different oxidation states although structural and magnetic characterization of the complexes bearing this ligand suggest that they can be mostly found as $\mathrm{M}^{\mathrm{IV}}$ (Cat-N-SQ)2, $\mathrm{M}^{\prime \prime \prime}(\mathrm{Cat}-\mathrm{N}-\mathrm{SQ})(\mathrm{Cat}-\mathrm{N}-\mathrm{BQ})$ and $\mathrm{M}^{\prime \prime}(\mathrm{Cat}-\mathrm{N}-\mathrm{BQ})_{2}$. The first complex of this family reported to exhibit VT was complex [Co"'(Cat-N-BQ)(Cat-N-SQ)]. ${ }^{[549]}$ Although first reported in 1988 by Pierpont et al., no evidence for tautomeric interconversion was shown to take place at that time. ${ }^{[550]} \mathrm{A}$ few years later the interconversion was also shown to take place in solid state though at much higher temperatures, ${ }^{[551]}$ and even the coexistence of at least two different IET phenomena, ligand-to-metal and ligand-to-ligand. ${ }^{[552]}$

$$
\left[\mathrm{Co}^{\prime \prime \prime}(\mathrm{Cat}-\mathrm{N}-\mathrm{BQ})(\mathrm{Cat}-\mathrm{N}-\mathrm{SQ})\right] \rightleftharpoons\left[\mathrm{Co}{ }^{\prime \prime}(\mathrm{Cat}-\mathrm{N}-\mathrm{BQ})_{2}\right]
$$

Afterwards, valence tautomerism has also been reported for the related manganese complex $\left[\mathrm{Mn}^{\mathrm{IV}}(\mathrm{Cat}-\mathrm{N}-\mathrm{SQ})_{2}\right]{ }^{[553]}$ Whereas its solid-state structural features are in agreement with the electronic distribution that involves the manganese atom in its oxidation state (IV), its solution temperature-dependence shows that this complex exhibits valence tautomerism involving three different tautomeric isomers associated to the three different oxidation states of the manganese ion: $\mathrm{Mn}(\mathrm{IV}), \mathrm{Mn}(\mathrm{III})$ and $\mathrm{Mn}(\mathrm{II})$. The advantages of valence tautomeric Schiff base complexes over transition metal complexes with o-quinone ligands are considerable. First, valence tautomeric Schiff base complexes display higher stabilities relative to atmospheric oxygen atmosphere in solution and solid state. Second, the differences between the optical properties of isomers involved in the valence tautomerism of the cobalt Schiff base complex are enhanced when compared to those observed for cobalt complexes with o-quinone ligands. Third, the Schiff base ligand exhibits a richer electrochemical behavior since it can exist in 
different oxidation forms, ranging from +1 to -3 , which may lead to stable coordination complexes with several metal ions in a variety of oxidation states.

The redox activity of the ligands not only allows for the existence of valence tautomerism but also for additional switching capacities resulting from an electrochemical process. Such a strategy has been shown to be very useful for the systematic tuning of the critical temperature at which there are equal amounts of both tautomers, as well as to establish arrays of several different states depending on the redox possibilities of the metal ion involved in the array. The first example of redox-tuned valence tautomerism was based on the reduction of complex [Co'"'(3,5-DTBCat)(3,5-DTBSQ)(bpy)]. ${ }^{[554]}$ Interestingly, the resulting reduced complex also exhibited a temperature-dependence consistent with the existence of a valence-tautomeric equilibrium. This fact allowed for the first time to establish an array of four states showing different optical and magnetic ground states controlled by two temperature-controlled valence tautomeric equilibria and two reversible redox processes. The possibility of entering the cycle at each state and advancing through the square array in a clockwise and counterclockwise direction was also established. Following this approach, Rovira et al., ${ }^{[555]}$ also established an array of four states based on the oxidation of the tautomeric complex [Co"'(Cat$\mathrm{N}-\mathrm{BQ})$ (Cat-N-SQ)]. Interestingly, the variable-temperature absorption spectra of the charged species [Co'l'(Cat-N-BQ $\left.)_{2}\right]^{+}$chemically formed upon partial oxidation showed a equilibrium consistent with a temperature-induced valence tautomerism shown in Equation 3. This fact allowed the establishing of an array of four states showing different optical and magnetic ground states by using a reversible oxidation process as additional stimulus.

$$
\left[\mathrm{CO}^{\prime \prime \prime}(\mathrm{Cat}-\mathrm{N}-\mathrm{BQ})_{2}\right]^{+} \rightleftharpoons[\mathrm{Co}(\mathrm{Cat}-\mathrm{N}-\mathrm{BQ})(\mathrm{SQ}-\mathrm{N}-\mathrm{BQ})]^{+}
$$

It is also important to emphasize that the presence of VT in a given complex has been traditionally associated almost exclusively to the role played by the the redox-active ligand and the transition metal ion. However, the existence of several other factors that can modulate the observation of VT has also been detected along all these years of research in the field. ${ }^{[556]}$ In other words, the same complex may exhibit or not, or the VT process can be shifted to much higher temperatures, depending on other structural and environmental parameters such as nature of the counter-ligand, charge-induced and environmental effects. ${ }^{[557]}$ One of the approaches recently followed to avoid such critical effects on a rerproducible manner has been the development of VT polymeric nanoparticles that exhibit the same behaviour independently whether if they are suspendend in a solution, polymeric matrix or solid state. In this sense, Ruiz-Molina et al. demonstrated that precipitation/coordination polymerization is a suitable approach for the fabrication of pre-synthesized units into functional metal-organic nanoparticles. Size-tunable nanoparticles that exhibit valence tautomerism have been obtained by using electroactive [Co'l'(3,5-dbsq)(3,5-dbcat)] units and the appropriate bridging ligand. Such a bridging ligand or polymerizing agent was previously shown by others to be crucial to the formation of nanoparticles. Whereas the bix ligand yields amorphous nanoparticles, the 4,4'-bipyridine ligand yields a crystalline material. The high flexibility of this strategy and its scalability to several different functional molecular materials will certainly expand the synthesis of VT materials with novel morphologies and working ranges by simple polymerization of functional units/clusters and metal ions with the appropriate bridging ligands. ${ }^{[58]}$

Finally, the comprehension and understanding of the characteristic features for all these VT complexes has been developed in parallel over the last decades, rising out a large interest in 
the scientific community as demonstrated by the large amount of examples developed for each case. From a fundamental approach, VT complexes represent a unique scenario to study IET in model molecular materials involving both redox-active Ligands and transiton metal ions. From an applied perspective, the large changes in the optical, structural, and magnetic properties that often accompany the valence tautomeric interconversion have potential applications in the development of molecular electronic devices that can be used as optical and/or magnetic data storage media.

\subsubsection{Physiological functions and related models}

Transition metal complexes where the internal electronic distribution may be modulated by different external stimuli are of interest not only for the field of valence tautomerism but also for several other fields such as the study of complexes bearing non-innocent ligands that perform physiological functions on biological systems. In this sense, transition metal complexes in which one or more of the ligands is present as a phenoxyl radical have attracted much interest due to their occurrence in enzymatic metalloproteins such as galactose oxidase $(\mathrm{GAO})^{[559]}$ or glyoxal oxidase (GLO). ${ }^{[560]}$ Other examples of such electronic lability in Nature involves the $\mathrm{Cu}(\mathrm{I}) / \mathrm{Cu}$ (II) pair found in a copper containing enzyme that catalyzes the oxidation of amines to aldehydes, ${ }^{[561]}$ an important process in relevant biological functions such as growth regulation and tissue maturation. Model studies have also been done by Speier et al., who described the existence of valence tautomerism for the species $\left[\mathrm{Cu}(\mathrm{py})_{2}(\mathrm{PhenQ})_{2}\right]^{[562]}$ and Kaim et al., who reported the use of a weak $\pi$-acceptor thioether in a $\mathrm{Cu}(\mathrm{I})$-semiquinone complex to induce valence tautomerism as a paramagnetic compound associated to amine oxidase enzymes. ${ }^{[563]}$ The acceptor characteristics of the counterligand in a series of copperquinone synthetic model with the general formula $\left[\left(Q^{n-}\right) C^{n+} L\right]$ have also been studied. Strong $\pi$ acceptor ligands such as $L=C O, C N R, \mathrm{PR}_{3}$ or $\mathrm{AsR}_{3}$ stabilize the $\mathrm{Cu}(\mathrm{I})$-semiquinone form whereas the use of non- $\pi$-acceptor ligands, such as amine ligands, favour the $\mathrm{Cu}(I I)$ semiquinone form. ${ }^{[564]}$ For instance, Buchanan et al., ${ }^{[565]}$ have demonstrated the possibility to modulate the charge distribution between the two electronic isomers $\left[\mathrm{L}_{2} \mathrm{Cu}\right.$ (1) 3,5 -DTBCat)] and $\left[\mathrm{L}_{2} \mathrm{Cu}(3,5-\mathrm{DTBSQ})\right]$ by changing the nature of the counter-ligand from a strong nitrogen donor to a soft phosphine donor. On the other side, Kaim et al., ${ }^{[566]}$ have also reported the use of a weak $\pi$-acceptor thioether to induce sensitive valence tautomerism in paramagnetic copper complexes related to amine oxidase enzymes. In a more recent study, the comparison between two thioether ligands that differ on their $\pi$-acceptor capacity and structural rigidity has been used to have a comparative study of both effects. ${ }^{[567]}$

Another nice example for the relevance of this molecular systems is their influence on the catalytic center of citochrome c oxidase ( $\mathrm{CcO}$ ). The substitution of the tyrosinate cross-linked for a semiquinone group have generated a long-lived imidazolyl-phenoxyl radical. ${ }^{[568]}$ The resulting complexes with the general formula (Cu-imidazolyl-phenoxyl) are created to mimic the $\mathrm{Cu}_{\mathrm{B}}{ }^{\prime \prime}$-histidine-tyrosinate center in an attempt to study its catalytic potential on biological processes. A nice example of the formation of such mimic models is complex [(tpa) $\mathrm{Cu}(\mathrm{Im}$ $\left.h q^{*} \mathrm{H}_{2}\right)$ ](OTs) $)_{2}$ complex. The study of its double deprotonation show the evidence of the free radical anion $\left(I m-s q^{*-}\right)$ and the presence of a valence tautomerism on the coordinative labile copper(I) intermediate. 


\subsection{Dye-sensitized solar cells.}

Catechol has long been known for its strong affinity for metal ions with high oxidation states and/or high charge to metal ion radius ratios, such as Fe'". In this regard, catechol shows a comparably high affinity for $\mathrm{Ti}^{\mathrm{iV}}{ }^{[569]}$ which would make dihydroxyaryl moieties, and enediols in general, excellent choices as robust anchors for photosensitizing dyes in Grätzel's $\mathrm{TiO}_{2}-\mathrm{NP}$ based dye-sensitized solar cells (DSSCs). ${ }^{[570]}$ Anchorage of the catechol molecule to $\mathrm{TiO}_{2}$ surfaces has been subject to detailed experimental research, ${ }^{[15,571,572]}$ showing that it is capable

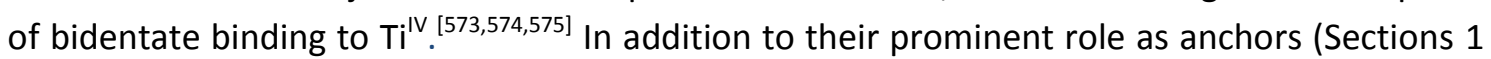
to 4), catechol moieties play an additional role as small-molecule sensitizers, playing a central role in the performance of the DSSC. ${ }^{[576]}$ In this regard, catechol has been shown to afford a very strong electronic coupling between the chromophore and the semiconductor, which is in contrast to those of weaker binding moieties, such as carboxy or phosphate groups. ${ }^{[577}$ This strong coupling manifests itself in the appearance of a new absorption band at around $>400$ $\mathrm{nm}$ in colloidal $\mathrm{TiO}_{2}$-catechol complexes, ${ }^{[571,575,578]}$ also observed in other $\mathrm{TiO}_{2}$-enediol complexes. ${ }^{[579]}$ Recent semiempirical, ${ }^{[580]}$ and $a b$ initio calculations ${ }^{[581,582]}$ have shown that this band may be assigned to a photoinduced electron transfer (ET) from the HOMO $-\pi$, dyelocalized- orbital to a hybrid orbital in the conduction band $(C B)$ of the semiconductor that participates both of catechol and $\mathrm{Ti}(3 \mathrm{~d})$ orbitals, and lies below the dye-localized, $\pi^{*}$ excited state. ${ }^{[580,574]}$ Therefore, whereas for so-called Type I DSSCs, electrons are first promoted upon photoexcitation to a dye-localized excited state, and subsequently injected into the conduction band of the semiconductor, in so-called Type II DSSCs, -such as catechol- $\mathrm{TiO}_{2}$ complexes-, electrons may be injected as well directly into the $\mathrm{CB}$ of the semiconductor. ${ }^{[580583]}$ Although such a direct injection mechanism should be, in principle, extremely fast, ${ }^{[584,575]}$ and theoretically $100 \%$ efficient, ${ }^{[583]}$ the strong electronic coupling may be also conducive to competitive charge recombination -or back electron transfer-, which dramatically decreases the net, dye-to-semiconductor ET efficiency. ${ }^{[583]}$

Several studies reporting the use of catecholic anchors/sensitizers have been published in the past years, in which catecholate sensitizer/anchors have been coupled to a variety of semiconductors - mainly $\mathrm{TiO}_{2},{ }^{[585,576,586]}$ but also $\mathrm{ZrO}_{2}{ }^{[587]} \mathrm{ZnO}^{[588,589]}$ and $\mathrm{NiO}-.^{[590]}$ In all cases, these reports evinced a difficult compromise between strong electron coupling and concomitant detrimental back electron transfer. Ward and co-workers already showed that the grafting properties of catechol-Ru complexes to $\mathrm{TiO}_{2}$ surfaces were better that those of similar, high-efficiency Ru-based dyes featuring carboxylic acid groups as anchoring moieties, which are more prone to desorption from the NP surface, particularly at acidic pHs. Nevertheless, lower photocurrents and overall electron transfer efficiencies were obtained for the catechol-anchored dyes in comparison, which was then attributed to aggregation of dye molecules on the metal oxide surface. ${ }^{[585]}$ Ghosh, Das and co-workers have investigated in detail the photoexcitation and relaxation kinetics of several catechol-anchored metallorganic dyes, reporting first on a Ru"-terpy dye connected to a catechol moiety by means of a conjugated double bond. The authors observed fast back electron transfer in spite of an ultrafast electron injection, leading to lower overall efficiency. ${ }^{[576]}$ When Os was used instead of Ru, direct excitation to triplet states with lower energy was observed, which enabled the extension of the excitation range to the red region of the solar spectrum, ${ }^{[587]}$ and led to slower charge recombination. ${ }^{[591]}$ Following Grätzel's approach for the "black dye", SCN ligands were also incorporated in $\mathrm{Ru}^{\prime \prime}$-bipy-catecholate- $\mathrm{TiO}_{2}$ complexes, which extended light absorption into the NIR range. ${ }^{[586]}$ The strong electronic coupling afforded by catecholate moieties was 
further evinced when solvatochromic ligands, such as $\mathrm{CN}$, where used in catechol-bound Ru" complexes; the solvatochromic effect dramatically diminished. ${ }^{[522]}$ Recently, the same authors introduced a dimethylaminophenyl substituent as $\pi$-electron donor in one of the bipy ligands of a polypy-Ru"-catechol dye, aimed at facilitating delocalization of the positive charge in the $\mathrm{Ru}^{\prime \prime}$-ligand sphere upon electron transfer, thus contributing to a greater spatial charge separation, and in turn to slower back electron transfer dynamics. With regard to the original $\mathrm{Ru}$ "-catechol complex, the dimethylaminophenyl-substituted complex showed a $20 \%$ higher efficiency in the electron injection yield, without impairment of the electronic coupling of the dye to the $\mathrm{TiO}_{2}$ nanoparticles. The beneficial effect of the ligand disappeared at vey low $\mathrm{pHs,}$ which was explained by the fact that the amino group responsible for the charge delocalization becomes protonated in these conditions, giving further proof of its role in the increase of charge separation. ${ }^{[593]}$ Similar results have been recently observed for Ru"-dye-catecholate complexes with other conjugated electron-donating moieties. ${ }^{[594]}$

Yoon and co-workers reported a notable increase in net injection efficiency through the design of ligands with conjugated electron-donating groups for Ru"-dye-catecholate complexes, ${ }^{[383]}$ to which similar studies by other groups followed. ${ }^{[593,594]}$ Another approach involved coating of the semiconductor NPs with a nanometer-thick $\mathrm{SrTiO}_{3}$ shell barrier, ${ }^{[595]}$ which led to a $70 \%$ improvement in charge collection at the interface. Finally, Burn, Meredith and co-workers prepared thiophene-catechol sensitizing moieties with 1, 2 and 3 conjugated thiophene units. Although conversion efficiencies increased slightly with the extension of the conjugation, the authors observed that charge recombination depended strongly on dye loading, the former being more severe as dye surface coverage the $\mathrm{TiO}_{2}$ NPs increased. ${ }^{[596]}$ Theoretical calculations on such systems suggested that the extension of the conjugation should enhance the cell efficiency due to an increase in light absorption, despite a lesser increase in opposing charge recombination. ${ }^{[597]}$

In addition to Ru"-dye complexes, catechol-anchored porphyrin dyes have been studied, showing a rather complex behavior, ${ }^{[598]}$ which was notably affected by the degree and type of aggregation between porphyrin units in solution. ${ }^{[599]}$ Particularly, photoexcitation decay for aggregates was found to be much slower than those of single, unaggregated dye molecules on $\mathrm{TiO}_{2}$ NPs. This was attributed to the stacking of porphyrin units, which effectively delocalized the positive charge after the electron transfer, separating it from the electron (negative charge) injected on the $\mathrm{TiO}_{2}$ surface, and thus slowing down the back electron transfer process. $^{[600]}$ Other catechol-like systems investigated include alizarin, [584, 601,581, 602 ] dopamine ${ }^{[603,604]}$ and, from a theoretical standpoint, anthraquinone and xanthene dyes with catechol groups. ${ }^{[605]}$ Despite its catecholic structure and bidentate hydroxyl-mediated binding to the $\mathrm{TiO}_{2}$ surface, alizarin did not give rise to direct electron injection in the conduction band of the semiconductor, likely due to the spread of the $\pi^{*}$ orbital over a greater molecular area, and thus a lower mixing of this dye excited state with Ti orbitals. ${ }^{[577,582]}$ On the other hand, electron injection to the conduction band ( $6 \mathrm{fs}$ ) was among the fastest ever recorded for any DSSC, ${ }^{[584]}$ which was explained by the proximity of the dye photoexcited state to the lower edge of the conduction band of the semiconductor. ${ }^{[602]}$

Hirsch, Peukert and co-workers reported the modification of $\mathrm{ZnO}$ nanoparticles with a $\mathrm{Zn}$ porphyrin, attached to the NP surface through two different catechol-based spacers. It was shown that catechol groups communicate electronically the porphyrin moiety and the ZnO NP, so that the porphyrin-based coating served as efficient fluorescence quencher of the $\mathrm{ZnO}$ 
surface emission. ${ }^{[588]}$ In a follow-up study, the authors bound cationic dendrons with catechol anchor groups on the surface of $\mathrm{ZnO}$ quantum dots, which were thus successfully modified to avoid aggregation. These QDs were further incorporated as mesoporous films in a proof-ofconcept DSSC design by coupling them to oppositely-charged porphyrins. ${ }^{[589]}$

\subsection{Miscellaneous devices.}

Nuckolls and co-workers reported that 1,2-dihydroxytetracene self-assembles on aluminum oxide by spontaneously forming dense $\pi$ - $\pi$ stacks, perpendicularly oriented to the surface and anchored through the catecholic bidentate end. The tetracene monolayer was shown to make up an active channel for hole transport in a nanoscale field-effect transistor device, with the $\mathrm{Al}_{2} \mathrm{O}_{3}$ surface acting as dielectric layer. ${ }^{[606]}$ Pickup et al. designed an asymmetrical supercapacitor, in which an anthraquinone-modified carbon (negative) electrode was coupled to a catecholdiazonium-modified carbon (positive) electrode, and separated by a dielectric perfluorinated membrane. This capacitor was shown to double the energy density of an analogous, carbon-based symmetrical capacitor, while at the same time providing a lower-cost alternative, since the catechol derivative substituted for commonly used, but also more expensive Ru oxide electrodes. ${ }^{[607]}$

Ward and co-workers have investigated the electrochromic properties of catechol derivatives designed to have reversible and selective light absorption in the NIR region upon the application of suitable redox potentials (electrochromic windows). In a first report, the authors described the electrochromic properties of a Ru(bipy) ${ }_{2}(\mathrm{TCC})$ complex (TCC=tetrachlorocatecholate) that showed intense, electroreversible absorption in the NIR (ca. $1000 \mathrm{~nm}$ ). When a monolayer of this compound was deposited on a nanocrystalline Sb-doped tin oxide electrode and a switching potential was applied, the light absorption properties changed almost completely within ca. $1.5 \mathrm{s.}^{[608]}$ More recently, the electrochromic properties of two $\mathrm{Ru}(\text { bipy) })_{2}$ (HHTP) complexes (where HHTP is the symmetrical tricatecholic hexahydroxytriphenylene), differing only in their solubility in organic/aqueous media, were studied. These compounds exhibited a completely reversible electrochemical behavior affecting the HHTO moiety, and exceptionally intense transitions in the NIR (ca. $1100 \mathrm{~nm}$ ) when in the fully (tri-) semiquinonic state, which completely disappeared once the HHTP moiety was switched back to the fully reduced (i.e., tricatecholate) state. Films of these species were prepared on indium tin oxide-coated glass and shown to be able to switch their absorption in the NIR reversibly and relatively fast upon application of commutating electric potentials. For one of the two complexes, reversibility was maintained up to $83 \%$ after $24 \mathrm{~h}$ (> 4000 redox cycles) on account of its poorer solubility and, thus likely, a decreased loss of material during operation in an aqueous environment. ${ }^{[609]}$ Using a bis-catecholic ligand (tetrahydroxyfluorone) capable of simultaneously binding two Ru nuclei, Natali et al. fabricated a proof-of-concept device capable of resolving NIR light pulses as short as $20 \mu \mathrm{s} .{ }^{[610]}$

Suitable chelating agents may be used to alter the binding geometry of under-coordinated surface atoms in order to restore optimal, fully coordinated geometries. Comparison between the spectroscopic features of unchanged and modified surfaces allows the identification of structural defects localized on surface atoms. ${ }^{[611,612]}$ Using this approach, Chen, Rajh and coworkers chose dopamine to study in detail the surface defects of $\mathrm{Fe}_{2} \mathrm{O}_{3}$ nanoparticles by XANES (X-ray absorption near-edge structure) spectroscopy, and distinguish them from other defects 
arising from the interior of the nanoparticle. ${ }^{[613]}$ Recently, the same group investigated different catechol derivatives as surface modifiers of metal oxides $\left(\mathrm{TiO}_{2}, \mathrm{ZrO}_{2}, \mathrm{CeO}_{2}, \mathrm{Fe}_{2} \mathrm{O}_{3}\right)$ that were irradiated with laser light, showing that these ligands enhanced Raman scattering significantly. This effect was also observed in bioconjugated systems were a biomolecule of interest is linked to the oxide surface through a catechol moiety, hinting to the potential of these systems for biomedical assays. ${ }^{[614,615]}$

An example of switchable catechol-based films is that of oxidized catechol-chitosan conjugates studied by Payne and co-workers. ${ }^{[616]}$ It was shown that catechol-modified chitosan films, although redox-active, were not conducting but became so in the presence of redox mediators in solution $\left(\mathrm{Fc}(\mathrm{MeOH})_{2} ;\left[\mathrm{Ru}\left(\mathrm{NH}_{3}\right)_{6}\right]^{3+}\right)$. At fixed concentrations of the mediator, the $\mathrm{CV}$ signal intensity increased with increasing catechol content in the film (amplification factor), albeit not linearly, due to increasing cross-linking and concomitantly decreasing film permeability, while the electrochemical response showed partial rectification characteristics. Moreover, after depletion, the film's redox activity could be regenerated by immersing it in the proper mediator solution under anodic and cathodic conditions, which may thus be considered as switches for the redox activity of the film. ${ }^{[617]}$ In a recent study, this research group showed that catechol-chitosan films can be made to be $\mathrm{pH}$ and voltage-responsive, being able to trap redox mediator pairs such as $\mathrm{Fe}(\mathrm{CN})_{6}^{3-/ 4-}$ in acidic conditions and selectively releasing them at high $\mathrm{pHs}$ and/or negative electric potentials. ${ }^{618}$

Nadeau and co-workers reported the first example of a cell-based biosensor based on electron transfer between a small molecule (dopamine) and CdSe/ZnS quantum dots (QDs). Dopamine moieties with their catechol rings facing outwards were covalently attached to QDs with different fluorescent emission patterns, and fluorescent quenching arising from charge transfer by dopamine species studied. Cells bearing specific receptors for dopamine were able to uptake selectively dopamine-functionalized QDs, which showed strong fluorescence in oxidizing intracellular environments, and quenched fluorescence in reducing ones. In these cases, fluorescence could be externally switched on by UV irradiation. Because of the inherent cytotoxicity of quinoid moieties, catechol-functionalized QDs exemplify the potential use of electro-donor/QD conjugates in QD photodynamic therapy. ${ }^{619}$ In a conflicting, more recent study, Medintz et al. studied the influence of $\mathrm{pH}$ on the photoluminiscence (PL) of similar dopamine-peptide CdSe/ZnS QD conjugates. The authors found that an increasing $\mathrm{pH}$ correlated with greater PL quenching in aerated environments, arising from the oxygenmediated oxidation of the dopamine moiety in basic media. This observation was replicated in a biological environment by microinjecting $\mathrm{pH}$-buffered solutions of functionalized QDs in cells, where cytosolic $\mathrm{pH}$ could be thus related to normalized PL down to the single cellular level. In this case, then it was dopamine (instead of the corresponding quinone) the chemical species responsible for QD photoluminiscence. ${ }^{620}$ In the latest, detailed study on the PL quenching mechanism of dopamine-QD conjugates, ZnS-overcoated CdSe QDs were capped with polymer DHLA-PEG bearing certain number of dopamine pending groups. Conjugates showed increasing photoluminescence quenching in alkaline media that unequivocally correlated with the presence of oxygen in solution leading to the formation of a certain amount of quinoid species. PL quenching was attributed to the annihilation of the QD excited state through a combination of two electron transfer mechanisms, - from high-potential dopamine moieties to the valence band of the $Q D$, as well as from the conduction band of the semiconductor to lowpotential quinone acceptors. ${ }^{621}$ Finally, Santra and co-workers studied the covalent modification of CdS:Mn/ZnS QDs with outward-facing, thiol-coupled dopamine moieties and 
their potential use as sensors for glutathione. In absence of the analyte, the authors observe fluorescence quenching of the functionalized QDs, which is attributed to the presence of at least a fraction of quinoid (oxidized) species. When glutathione is added to the medium, the covalent bond between the dopamine/quinone layer and the QDs is severed, and the fluorescence of the latter restored. ${ }^{622}$ 


\section{Conclusions and Outlook.}

In terms of the number of academic and technological proof-of-concept studies published in the past years, catechol derivatives as key components of adhesives and bioadhesives have a very promising outlook. In this respect, not only do these materials already offer bioinspired alternatives with performance comparable to that of commercial super-glues, but also, biocompatible variants capable of adhesion in aqueous environments and with low immflamatory response. On the other hand, the versatility of polydopamine as easy-to-use universal primer has already spun a virtual explosion of scientific work that will no doubt keep on growing in the near future. Also promising is the quest for functional materials with more robust adhesive catecholic anchors, which should increase their chances at becoming commercially viable as functional coatings.

Catechols still have much to offer in other areas, especially in those where it acts as a stimulus responsive material. One of the key points so far has been the control of the reversibility of the redox character of the catechol/quinone pair, which is obviously essential to achieve durable switching behavior. Whenever catechols manifest their messy, irreversible reactivity, they still present some interesting challenges, such as the final elucidation of the structure of melanins, which despite much effort, still remains elusive. 


\section{Bibliography.}

[1] E. S. Bromberg-Martin, M. Matsumoto, O. Hikosaka, Neuron 2010, 68, 815.

[2] J.H. Waite, M.L. Tanzer, Science 1981, 212, 1038.

[3] V. V. Papov, T. V. Diamond, K. Biemann, J. H. Waite, J. Biol. Chem. 1995, 270, 20183.

[4] J.H. Waite, Int. J. Adhesion and Adhesives 1987, 7, 9.

[5] T.J. Deming, Curr. Opin. Chem. Biol. 1999, 3, 100.

[6] J.H. Waite, Integr. Comp. Biol. 2002, 42, 1172.

[7] J.H. Waite, Biol. Bull. 1992, 183, 178.

[8] R. J. Stewart, T. C. Ransom, V. Hlady, J. Pol. Sci. B: Pol. Phys. 2011, 49, 757.

[9] H. Yamamoto, Biotechnol. Genet. Eng. 1995, 13, 133.

[10] B.P. Lee, P.B. Messersmith, J.N. Israelachvili, J.H.Waite, Annu. Rev. Mater. Res. 2011, 41, 99.

[11] J.H. Waite, T.J. Housley, M.L. Tanzer, Biochemistry 1985, 24, 5010.

[12] J. H. Waite, R. A. Jensen, D. E. Morse, Biochemistry 1992, 31, 5733.

[13] H. Lee, N. F. Scherer, P. B. Messersmith, Proc. Natl. Acad. Sci. USA 2006, 103, 12999.

[14] J. Wang, M. N. Tahir, M. Kappl, W. Tremel, N. Metz, M. Barz, P. Theato, H. J. Butt, Adv. Mater. 2008, 20,3872 .

[15] S.C. Li, L.N. Chu, X.Q. Gong, U. Diebold, Science 2010, 328, 882.

[16] M. B. McBride, L. G. Wesselink, Environ. Sci. Technol. 1988, 22, 703.

[17] L.A. Burzio, J.H. Waite, Biochemistry 2000, 39, 11147.

[18] M.E. Yu, J.Y. Hwang, T.J. Deming, J. Am Chem. Soc. 1999, 121, 5825.

[19] T. H. Anderson, J. Yu, A. Estrada, M. U. Hammer, J. H. Waite and J. N. Israelachvili, Adv. Funct. Mater. 2010, 20, 4196.

[20] J. Yu , W. Wei , E. Danner , J.N. Israelachvili , J.H. Waite, Adv. Mat. 2011, 23, 2362.

[21] J.H. Waite, X. Qin, Biochemistry 2001, 40, 2887.

[22] J.D. White, J.J. Wilker, Macromolecules 2011, 44, 5085.

[23] M.J. Whirter, P.J. Bremer, I.L. Lamont, A.J. Quillan, Langmuir 2003, 19, 3575.

[24] H.G. Upritchard, J. Yang, P.J. Bremer, I.L. Lamont, A.J. McQuillan, Langmuir 2011, 27, 10587. 
[25] J.I. Williams, H.R. Bhattacharjee, I. Goldberg, A.J. Salerno, M.D. Swerdlof, P.O. Unger, Synthesis of High Molecular Weight Peptide Polymers and Copolymers Containing L-Dopa Residues (Final Report to the Department of the Navy - Office of Naval Research), 1988.

[26] M.J. Sever, J.J. Wilker, Tetrahedron 2001, 57, 6139.

[27] M. Yu, T.J. Deming, Macromolecules 1998, 31, 4739.

[28] G. Westwood, T.N. Horton, J.J. Wilker, Macromolecules 2007, 40, 3960.

[29] B.P. Lee, J.L. Dalsin, P.B. Messersmith, Biomacromolecules 2002, 3, 1038.

[30] B.P. Lee, C.-Y. Chao, F.N. Nunalee, E. Motan, K.R. Shull, P.B. Messersmith, Macromolecules 2006, 39, 1740.

[31] M. Guvendiren, D.A. Brass, P.B. Messersmith, K.R. Shull, J. Adhesion 2009, 85, 631.

[32] N. Holten-Andersen, M.J. Harrington, H. Birkedal, B.P. Lee, P.B. Messersmith, K.Y.C. Lee, J.H. Waite, Proc. Natl. Acad. Sci. USA 2011, 108, 2651.

[33]J. Monahan, J.J. Wilker, Chem. Commun. 2003, 1672.

[34] B.P. Lee, K. Huang, F.N. Nunalee, K.R. Shull, P.B. Messersmith, J. Biomat. Sci. - Pol. Ed. 2004, 15, 449.

[35] H. Chung, P. Glass, J.M. Pothen, M. Sitti, N.R. Washburn, Biomacromolecules 2011, 12, 342.

[36] J. Saiz-Poseu, I. Alcón, R. Alibés, F. Busqué, J. Faraudo, D. Ruiz-Molina CrystEngComm, 2012, 14, 264.

[37] J. Saiz-Poseu, J. Faraudo, A. Figueras, R. Alibes, F. Busqué, D. Ruiz-Molina Chem. Eur. J. 2012, 18, 3056.

[38] Q. Ye, F. Zhou, W. Liu, Chem. Soc. Rev. 2011, 40, 4244.

[39] K. Yamada, T. Chen, G. Kumar, O. Vesnovsky, L.D.T. Topoleski, G.F. Payne, Biomacromolecules 2000, $1,252$.

[40] C. R. Matos-Pérez, J. D. White, J. J. Wilker, J. Am. Chem. Soc. 2012, 134, xxxx.

[41] P. Podsiadlo, Z. Liu, D. Paterson, P.B. Messersmith, N.A. Kotov, Adv. Mater. 2007, 19, 949.

[42] L.M. Hamming, X.W. Fan, P.B. Messersmith, L.C. Brinson, Compos. Sci. Technol. 2008, 68, 2042.

[43] H. Lee, B.P. Lee, P.B. Messersmith, Nature 2007, 448, 338.

[44] P. Glass, H. Chung, N.R. Washburn, M. Sitti, Langmuir 2009, 25, 6607.

[45] P. Glass, H.Y. Chung, N.R. Washburn, M. Sitti, Langmuir 2010, 26, 17357.

[46] H. Lee, Y. Lee, A.R. Statz, J. Rho, T.G. Park, P.B. Messersmith, Adv. Mater. 2008, 20, 1619.

[47] S. Ryu, Y. Lee, Y.W. Hwang, S. Hong, C. Kim, T.G. Park, H. Lee, S.H. Hong, Adv. Mater. 2011, $23,1971$.

[48] T.J. Deming, Prog. Polym. Sci. 2007, 32, 858. 
[49] C.E. Brubaker, H. Kissler, L.J. Wang, D.B. Kaufman, P.B. Messersmith, Biomaterials 2010, 31, 420.

[50] C.M. Haller, W. Buerzle, C.E. Brubaker, P.B. Messersmith, E. Mazza, N. Ochsenbein-Koelble, R. Zimmermann, M. Ehrbar, Prenat. Diagn. 2011, 31, 654.

[51] J.L. Murphy, L. Vollenweider, F.M. Xu, B.P. Lee, Biomacromolecules 2010, 11, 2976.

[52] M. Brodie, L. Vollenweider, J.L. Murphy, F. Xu, A. Lyman, W.D. Lew, B.P. Lee, Biomed. Mater. 2011, 6, 15014.

[53] H. Shao, R.J. Stewart, Adv. Mater. 2010, 22, 729.

[54] H. Shao, K.N. Bachus, R.J. Stewart, Macromol. Biosci. 2009, 9, 464.

[55] Y. Lee, H.J. Cheng, S. Yeo, C.-H. Ahn, H. Lee, P.B. Messersmith, T.G. Park, Soft Matter 2010, 6, 977.

[56] J.H. Ryu, Y. Lee, W.H. Kong, T.G. Kim, T.G. Park, H. Lee, Biomacromolecules 2011, 12, 2653.

[57] Y. Yao, K. Fukazawa, W. Ma, K. Ishihara, N. Huang, Appl. Surf. Sci. 2012, 258, 5418.

[58] S.W. Taylor, D.B. Chase, M.H. Emptage, M.J. Nelson, J.H. Waite, Inorg. Chem. 1996, 35, 7572.

[59] T.L. Coombs, P.J. Keller, Aquat. Toxicol. 1981, 1, 291.

[60] S.W. Taylor, G.W. Luther III, J.H. Waite, Inorg. Chem. 1994, 33, 5819.

[61] M.J. Sever, J.T. Weisser, J. Monahan, S. Srinivasan, J.J. Wilker, Angew. Chem. Int. Ed. 2004, 43, 448.

[62] J.J. Wilker, Current Opinion in Chemical Biology 2010, 14, 276.

[63] E. Loizou, J.T. Weisser, A. Dundigalla, L. Porcar, G. Schmidt, J.J. Wilker, Macromol. Biosci. 2006, 6, 711.

[64] Q. Lin, D. Gourdon, C.J. Sun, N. Holten-Andersen, T.H. Anderson, J.H. Waite, J.N. Israelachvili, Proc. Natl. Acad. Sci. U. S. A. 2007, 104, 3782.

[65] H. Zeng, D.S. Hwang, J.N. Israelachvili, J.H. Waite, Proc.Natl. Acad. Sci. USA 2010, 107, 12850.

[66] M.J. Harrington, A. Masic, N. Holten-Andersen, J.H. Waite, P. Fratzl, Science 2010, 328, 216.

[67] N. Holten-Andersen, G.E. Fantner, S. Hohlbauch, J.H. Waite, F.W. Zok, Nat. Mater. 2007, 6, 669.

[68] P.B. Messersmith, Science 2010, 328, 180.

[69] A. Doraiswamy, T.M. Dunaway, J.J. Wilker, R.J. Narayan, J. Biomed. Mater. Res. 2009, 89B, 28.

[70] Z. Shafiq, J. Cui, L. Pastor-Pérez, V. SanMiguel, R. A. Gropeanu, C. Serrano, A. del Campo, Angew. Chem. Int. Ed. 2012, 51, 4332.

[71] L. He, D.E. Fullenkamp, J.G. Rivera, P.B. Messersmith, Chem. Commun. 2011, 47, 7497.

[72] H. Lee, S.M. Dellatore, W.M. Miller, P.B. Messersmith, Science 2007, 318, 426.

[73] J.H. Waite, Nat. Mater. 2008, 7, 8. 
[74] US 2008/0149566 A1

[75] H. Lee, J. Rho, P.B. Messersmith, Adv. Mater. 2009, 21, 431.

[76] L.Q. Xu, W.J. Yang, K.G. Neoh, E.T. Kang, G.D. Fu, Macromolecules 2010, 43, 8336.

[77] S.M. Kang, S. Park, D. Kim, S.Y. Park, R.S. Ruoff, H. Lee, Adv. Funct. Mater. 2011, 21, 108.

[78] F. Bersmann, A. Ponche, C. Ringwald, J. Hemmerlé, J. Raya, B. Bechinger, J.-C. Voegel, P. Schaaf, V. Ball, J. Phys. Chem. C 2009, 113, 8234.

[79] D. R. Dreyer, D. J. Miller, B. D. Freeman, D. R. Paul, C. W. Bielawski, Langmuir 2012, 28, 6428.

[80] S. Kang, J. Rho, I.S. Choi, P.B. Messersmith, H. Lee, J. Am. Chem. Soc. 2009, 131, 13224.

[81] C. Tao, S. Yang, J. Zhang, J. Wang, Appl. Surf. Sci. 2009, 256, 294.

[82] H.Y. Hu, B. Yu, Q. Ye, Y.S. Gu, F. Zhou, Carbon 2010, 48, 2347.

[83] S. Hong, K.Y. Kim, H.J. Wook, S.Y. Park, K.D. Lee, D.Y. Lee, H. Lee, Nanomedicine 2011, 6, 793.

[84] S.H. Ku, C.B. Park, Biomaterials 2010, 31, 9431.

[85] Y.M. Shin, H. Park, H. Shin, Macromol. Res. 2011, 19, 835.

[86] S.H. Yang, S.M. Kang, K.B. Lee, T.D. Chung, H. Lee, I.S. Choi, J. Am. Chem. Soc. 2011, 133, 2795.

[87] Q. Ye, X.L. Wang, H.Y. Hu, D.A. Wang, S.B. Li, F. Zhou, J. Phys. Chem. C 2009, 113, 7677.

[88] I. You, S.M. Kang, Y. Byun, H. Lee, Bioconjugate Chem. 2011, 22, 1264.

[89] T.G. Kim, H. Lee, Y. Jang, T.G. Park, Biomacromolecules 2009, 10, 1532.

[90] J. Jiang, L. Zhu, X. Li, Y. Xu, B. Zhu, Journal of Membrane Science 2010, 364, 194.

[91] T.G. Kim, Y. Lee, T.G. Park, Int. J. Pharm. 2010, 384, 181.

[92] S.H. Ku, J.S. Lee, C.B. Park, Langmuir 2010, 26, 15104.

[93] A. Liu, L. Zhao, H. Bai, H. Zhao, X. Xing, G. Shi, ACS Appl. Mater. Interfaces 2009, 1, 951.

[94] S. Kang, M. Elimelech, Langmuir 2009, 25, 9656.

[95] K. Kang, I.S. Choi, Y. Nam, Biomaterials 2011, 32, 6374.

[96] H. Liu, P. Xi, G. Xie, Y. Shi, F. Hou, L. Huang, F. Chen, Z. Zeng, C. Shao, J. Wang, J. Phys. Chem. C 2012, $116,3334$.

[97] J.F. Ou, J.Q. Wang, S. Liu, J.F. Zhou, S.R. Yang, J. Phys. Chem. C 2009, 113, 20429.

[98] Y. Mi, Z. Wang, X. Liu, S. Yang, H. Wang, J. Ou, Z. Li, J. Wang, J. Mater. Chem. 2012, 22, 8036.

[99] T. Akter, W. S. Kim, ACS Appl. Mater. Interfaces 2012, 4, 1855.

[100] Q. Ye, H.Y. Hu, B. Yu, X.L. Wang, S.B. Li, F. Zhou, Phys. Chem. Chem. Phys. 2010, 12, 5480.

[101] W. Ye, D. Wang, H. Zhang, F. Zhou, W. Liu, Electrochimica Acta 2010, 55, 2004. 
[102] F. Li, L. Yang, C. Zhao, Z. Du, Anal. Methods 2011, 3, 1601.

[103] C.C. Lu, M. Zhang, A.J. Li, X.W. He, X.B. Yin, Electroanalysis 2011, 23, 2421.

[104] H-W. Chien, W-H Kuo, M-J Wang, S.-W. Tsai, W.-B. Tsai, Langmuir 2012, 28, 5775.

[105] K. Sun, Y. Xie, D. Ye, Y. Zhao, Y. Cui, F. Long, W. Zhang, X. Jiang, Langmuir 2012, 28, 2131.

[106] T.A. Morris, A.W. Peterson, M.J. Tarlov, Anal. Chem. 2009, 81, 5413.

[107] C. Tao, J. Zhang, S.J. Yang, Nanosci. Nanotechnol. 2011, 11, 5068.

[108] C.K. Poh, Z. Shi, T.Y. Lim, K.G. Neoh, W. Wang, Biomaterials 2010, 31, 1578.

[109] M. Lai, K. Cai, L. Zhao, X. Chen, Y. Hou, Z. Yang, Biomacromolecules 2011, 12, 1097.

[110] Y. Wan, D. Zhang, Y. Wang, P. Qia, B. Hou, Biosens. Bioelectron. 2011, 26, 2595.

[111] C.S.O. Paulo, M. Vidal, L.S. Ferreira, Biomacromolecules 2010, 11, 2810.

[112] M.E. Lynge, R. Ogaki, A.O. Laursen, J. Lovmand, D.S. Sutherland, B. Städler, ACS Appl. Mater. Interfaces 2011, 3, 2142.

[113] M.O. Avilés, C. Lin, M. Zelivyanskaya, J.G. Graham, R.M. Boehler, P.B. Messersmith, L.D. Shea, Biomaterials 2010, 31, 1140.

[114] K.J. Jeong, L. Wang, C.F. Stefanescu, M.W. Lawlor, J. Polat, C.H. Dohlman, R.S. Langerad, D.S. Kohane, Soft Matter 2011, 7, 8305.

[115] S. Yuan, D. Wan, B. Liang, S.O. Pehkonen, Y.P. Ting, K.G. Neoh, E.T. Kang, Langmuir 2011, 27, 2761.

[116] A. Nordberg, P. Antoni, M.I. Montanez, A. Hult, H.V. Holst, M. Malkoch, ACS Appl. Mater. Interfaces 2010, 2, 654 .

[117] Z. Iqbal, S. Alsudir, M. Miah, E.P.C. Lai, Electrophoresis 2011, 32, 2181.

[118] W. Zhou, S. Tang, Q. Yao, F. Chen, H. Yang, X. Wang, Biosens. Bioelectron. 2010, 26, 585.

[119] B. Xing, , X.B. Yin, PLOS ONE 2009, 4, e6451.

[120] Y. Tan, W. Deng, Y. Li, Z. Huang, Y. Meng, Q. Xie, M. Ma, S. Yao, J. Phys. Chem. B 2010, 114, 5016.

[121] Y. Fu, P. Li, L. Bu, T. Wang, Q. Xie, X. Xu, L. Lei, C. Zou, S. Yao, J. Phys. Chem. C 2010, 114, 1472.

[122] Y. Fu, P. Li, T. Wang, L. Bu, Q. Xie, X. Xu, L. Lei, C. Zou, J. Chen, S. Yao, Biosensors and Bioelectronics 2010, 25, 1699.

[123] H.O. Ham, Z. Liu, K.H.A. Lau, H. Lee, P.B. Messersmith, Angew. Chem. Int. Ed. 2011, 50, 732.

[124] E. Kim, I. T. Song, S. Lee, J-S Kim, H. Lee, J.-H. Jang, Angew. Chem. Int. Ed. 2012, 51, XXX.

[125] X. Wang, Q. Ye, T. Gao, J. Liu, F. Zhou, Langmuir 2012, 28, 2574.

[126] T. Chen, R. Vazquez-Duhalt, C.F. Wu, W.E. Bentley, G.F. Payne, Biomacromolecules 2001, 2, 456. 
[127] T. Chen, H.D. Embree, L.Q. Wu, G.F. Payne, Biopolymers 2002, 64, 292.

[128] L.Q. Wu, R. Ghodssi, Y.A. Elabd, G.F. Payne, Adv. Func. Mater. 2005, 15, 189.

[129] A.R. Statz, R.J. Meagher, A.E. Barron, P.B. Messersmith, J. Am. Chem. Soc. 2005, 127, 7972.

[130] J.L. Dalsin, L. Lin, S. Tosatti, J. Vörös, M. Textor, P.B. Messersmith, Langmuir 2005, 21, 640.

[131] A. Statz, J. Finlay, J. Dalsin, M. Callow, J.A. Callow, P.B. Messersmith, Biofouling 2006, 22, 391.

[132] X. Fan, L. Lin, J.L. Dalsin, P.B. Messersmith, J. Am. Chem. Soc. 2005, 127, 15843.

[133] K. Gademann, Y. Bethuel, Angew. Chem., Int. Ed. 2004, 43, 3327.

[134] J.Y. Wach, B. Malisova, S. Bonazzi, S. Tosatti, M. Textor, S. Zurcher, K. Gademann, Chem.-Eur. J. 2008, 14, 10579.

[135] K. Gademann, J. Kobylinska, J.Y. Wach, T.M. Woods, BioMetals 2009, 22, 595.

[136] J.Y. Wach, S. Bonazzi, K. Gademann, Angew. Chem., Int. Ed. 2008, 47, 7123.

[137] S. Saxer, C. Portmann, S. Tosatti, K. Gademann, S. Zurcher, M. Textor, Macromolecules 2010, 43, 1050.

[138] H. Lee, K.D. Lee, K.B. Pyo, S.Y. Park, H. Lee, Langmuir 2010, 26, 3790.

[139] T. Gillich, E.M. Benetti, E. Rakhmatullina, R. Konradi, W. Li, A. Zhang, A.D. Schlüter, M. Textor, J. Am. Chem. Soc. 2011, 133, 10940.

[140] C. Gao, G. Li, H. Xue, W. Yang, F. Zhang, S. Jiang, Biomaterials 2010, 31, 1486.

[141] A. Charlot, V. Sciannamea, S. Lenoir, E. Faure, R. Jerôme, C.C. Jerôme, C. Van De Weerdt, J. Martial, C. Archambeau, N. Willet, A.S. Duwez, C.A. Fustine, C. Detrembleur, J. Mater. Chem. 2009, 19, 4117.

[142] C $\quad-\quad$, E. Faure, T. Svaldo-Lanero, F. Farina, $\quad$, C. Van De Weerdt, J. Martial, A.

S. Duwez, C. Detrembleur, Langmuir 2012, 28, 7233.

[143] E. Faure, P. Lecomte, S. Lenoir, C. Vreuls, C. Van De Weerdt, C. Archambeau, J. Martial, C. Jerôme, A.S. Duwez, C. Detrembleur, J. Mater. Chem. 2011, 21, 7901.

[144] T. Shalev, A. Gopin, M. Bauer, R. W. Stark, S. Rahimipour, J. Mater. Chem. 2012, 22, 2026.

[145] H. Han, J. Wu, C.W. Avery, M. Mizutani, X. Jiang, M. Kamigaito, Z. Chen, C. Xi, K. Kuroda, Langmuir 2011, 27, 4010.

[146] H. Xu, X. Shi, H. Ma, Y. Lv, L. Zhang, Z. Mao, Appl. Surf. Sci. 2011, 257, 6799.

[147] C. Yang, G.L. Liang, K.M. Xu, P. Gao, B. Xu, J. Mater. Sci. 2009, 44, 1894.

[148] M. Sureshkumar, D.Y. Siswanto, C.K. Lee, J. Mater. Chem. 2010, 20, 6948.

[149] T. He, Z.L. Shi, N. Fang, K.G. Neoh, E.T. Kang, V. Chan, Biomaterials 2009, 30, 317. 
[150] X. Hua, K.G. Neoh, Z. Shi, E.T. Kang, C. Poh, W. Wang, Biomaterials 2010, 31, 8854.

[151] N.D. Brault, C. Gao, H. Xue, M. Piliarik, J. Homola, S. Jiang, Q. Yu, Biosens. Bioelectron. 2010, 25, 2276.

[152] D.C. Hansen, S.C. Dexter, J.H. Waite, Corros. Sci. 1995, 37, 1423.

[153] J. Ou, J. Wang, J. Zhou, S. Liu, Y. Yu, X. Pang, S. Yang, Prog. Org. Coat. 2010, 68, 244.

[154] E. Faure, E. Halusiak, F. Farina, N. Giamblanco, C. Motte, M. Poelman, C. Archambeau, C. V. de Weerdt, J. Martial, C. Jérome, A-S. Duwez, C. Detrembleur, Langmuir 2012, 28, 2971.

[155] J. Liu, J. Li, B. Yu, B. Ma, Y. Zhu, X. Song, X. Cao, W. Yang, F. Zhou, Langmuir 2011, 27, 11324.

[156] H. Zhang, H. Hu, W. Ye, F. Zhou, J. Appl. Polym. Sci. 2011, 122, 3145.

[157] S. Chen, Y. Chen, Y. Lei, Y. Yin, Electrochem. Commun. 2009, 11, 1675.

[158] M.H. Ryou, Y.M. Lee, J.K. Park, J.W. Choi, Adv. Mater. 2011, 23, 3066.

[159] B. Yu, J.X. Liu, S.J. Liu, F. Zhou, Chem. Commun. 2010, 46, 5900.

[160] S.M. Kang, I. You, W.K. Cho, H.K. Shon, T.G. Lee, I.S. Choi, J.M. Karp, H. Lee, Angew. Chem., Int. Ed. 2010, 49, 9401.

[161] I. You, S. M. Kang, S. Lee, Y. O. Cho, J. B. Kim, S. B. Lee, Y. S. Nam, H. Lee, Angew. Chem. Int. Ed. 2012, 51, XXX.

[162] M. Rodenstein, S. Zürcher, S.G.P. Tosatti, N.D. Spencer, Langmuir 2010, 26, 16211.

[163] X. Wang, Q. Ye, J. Liu, X. Liu, F. Zhou, J. Colloid Interface Sci. 2010, 351, 261.

[164] Q. Ye, X.L. Wang, S.B. Li, F. Zhou, Macromolecules 2010, 43, 5554.

[165] Q. Liu, X. Wang, B. Yu, F. Zhou, Q. Xue, Langmuir 2012, 28, 5845.

[166] X.B. Yin, D.Y. Liu, J. Chromatogr. A 2008, 1212, 130.

[167] J.T. Arena, B. McCloskey, B.D. Freeman, J.R. McCutcheon, J. Membrane Sci. 2011, 375, 55.

[168] Y. Zhang, N. Kohler, M. Zhang, Biomaterials 2001, 23, 1553.

[169] J. Xie, C.J. Xu, Z.C. Xu, Y.L. Hou, K.L. Young, S.X. Wang, N. Pourmond, S.H. Sun, Chem. Mater. 2006, $18,5401$.

[170] M.I. Shukoor, F. Natalio, H.A. Therese, M.N. Tahir, V. Ksenofontov, M. Panthöfer, M. Eberhardt, P. Theato, H.C. Schröder, W.E.G. Müller, W. Tremel, Chem. Mater. 2008, 20, 3567.

[171] Z. Yang, H. Gu, J. Du, J. Gao, B. Zhang, X. Zhang, B. Xu, Tetrahedron 2007, 63, 7349.

[172] H. M. Song, J.C. Kim, J.H. Hong, Y.B. Lee, J. Choi, J.I. Lee, W.S. Kim, J.H. Kim, N.H. Hur, Adv. Funct. Mater. 2007, 17, 2070. 
[173] E. Amstad, T. Gillich, I. Bilecka, M. Textor, E. Reimhult, Nano Lett. 2009, 9, 4042.

[174] L. Zhang, H. Xue, C. Gao, L. Carr, J. Wang, B. Chu, S. Jiang, Biomaterials 2010, 31, 6582.

[175] J. Xie, C. Xu, N. Kohler, Y. Hou, S. Sun, Adv. Mater. 2007, 19, 3163.

[176] K. Somaskandan, T. Veres, M. Niewczas, B. Simard, New J. Chem. 2008, 32, 201.

[177] Y. Lee, H. Lee, Y.B. Kim, J. Kim, T. Hyeon, H.W. Park, P.B. Messersmith, T.G. Park, Adv. Mater. 2008, 20, 4154 .

[178] K.H. Bae, Y.B. Kim, Y. Lee, J.Y. Hwang, H.W. Park, T.G. Park, Bioconjugate Chem. 2010, 21, 505.

[179] L. Wang, Z.M. Yang, J.H. Gao, K.M. Xu, H.W. Gu, B. Zhang, X.X. Zhang, B. Xu, J. Am. Chem. Soc. 2006, 128, 13358.

[180] H.W. Gu, K.M. Xu, Z.M. Yang, C.K. Chang, B. Xu, Chem. Commun. 2005, 4270.

[181] J.H. Gao, G.L. Liang, J.S. Cheung, Y. Pan, Y. Kuang, F. Zhao, B. Zhang, X.X. Zhang, E.X. Wu, B. Xu, J. Am. Chem. Soc. 2008, 130, 11828.

[182] J. Xie, K. Chen, H.Y. Lee, C.J. Xu, A.R. Hsu, S. Peng, X.Y. Chen, S.H. Su, J. Am. Chem. Soc. 2008, 130, 7542.

[183] B. Wang, C. Xu, J. Xie, Z. Yang, S. Sun, J. Am. Chem. Soc. 2008, 130, 14436.

[184] E. Amstad, J. Kohlbrecher, E. Muller, T. Schweizer, M. Textor, E. Reimhult, Nano Lett. 2011, 11, 1664.

[185] E. Amstad, A.U. Gehring, H. Fischer, V.V. Nagaiyanallur, G. Hahner, M. Textor, E. Reimhult, J. Phys. Chem. C 2011, 115, 683.

[186] B. Wang, J. Hai, Q. Wang, T. Li, Z. Yang, Angew. Chem., Int. Ed. 2011, 50, 3063.

[187] A. K. L. Yuen, G. A. Hutton, A. F. Masters, T. Maschmeyer, Dalton Trans. 2012, 41, 2545.

[188] M. D. Shultz, J. U. Reveles, S. N. Khanna, E. E. Carpenter, J. Am. Chem. Soc. 2007, 129, 2482.

[189] M. Chanana, S. Jahn, R. Georgieva, J.F. Lutz, H. Baumler, D. Wang, Chem. Mater. 2009, 21, 1906.

[190] C. Stefaniu, M. Chanana, D. Wang, D.V. Novikov, G. Brezesinski, H. Möhwald, ChemPhysChem 2010, 11, 3585.

[191] C. Stefaniu, M. Chanana, H. Ahrens, D. Wang, G. Brezesinski, H. Möhwald, Soft Matter 2011, 7, 4267.

[192] C. Stefaniu, M. Chanana, D. Wang, D.V. Novikov, G. Brezesinski, H. Mohwald, Langmuir 2011, 27, 1192.

[193] H.W. Gu, Z.M. Yang, J.H. Gao, C.K. Chang, B. Xu, J. Am. Chem. Soc. 2005, 127, 34. 
[194] C. Xu, J. Xie, D. Ho, C. Wang, N. Kohler, E.G.Walsh, J.R. Morgan, Y.E. Chin, S. Sun, Angew. Chem. Int. Ed. 2008, 47, 173.

[195] H. B. Na, G. Palui, J. T. Rosenberg, X. Ji, S. C. Grant, H. Mattoussi, ACS Nano 2012, 6, 389.

[196] C. Xu, K. Xu, H. Gu, R. Zheng, H. Liu, X. Zhang, Z. Guo, B. Xu, J. Am. Chem. Soc. 2004, 126, 9938.

[197] B. Wang, J. Hai, Z. Liu, Q. Wang, Z. Yang, S. Sun, Angew. Chem. Int. Ed. 2010, 49, 4576.

[198] T. Rajh, Z. Saponjic, J. Liu, N.M. Dimitrijevic, N.F. Scherer, M. Vega-Arroyo, P. Zapol, L.A. Curtiss, M.C. Thurnauer, Nano Lett. 2004, 4, 1017.

[199] J. Liu, L. de la Garza, L. Zhang, N.M. Dimitrijevic, X. Zuo, D.M. Tiede, T. Rajh, Chem. Phys. 2007, 339, 154.

[200] H. Lee, K. Lee, I.K. Kim, T.G. Park, Adv. Funct. Mater. 2009, 19, 1884.

[201] G. Wang, H. Huang, G. Zhang, X. Zhang, L. Wang, Anal. Methods 2011, 3, 2475.

[202] W.H. Zhou, C.H. Lu, X.C. Guo, F.R. Chen, H.H. Yang, X.R. Wang, J. Mater. Chem. 2010, 20, 880.

[203] R. Ouyang, J. Lei, H. Ju, Nanotechnology 2010, 21, 185502.

[204] M. Zhang, X. Zhang, X. He, L. Chen, Y. Zhang, Nanoscale 2012, 4, 3141.

[205] A.R. Studart, E. Amstad, L.J. Gauckler, Langmuir 2007, 23, 1081.

[206] B. Fei, B. Qian, Z. Yang, R. Wang, W.C. Liu, C.L. Mak, J.H. Xin, Carbon 2008, 46, 1792.

[207] Y.H. Wang, I. Zhitomirsky, Langmuir 2009, 25, 9684.

[208] K. Wu, I. Zhitomirsky, Int. J. Appl. Ceram. Technol. 2011, 8, 920.

[209] X.W. Fan, L.J. Lin, P.B. Messersmith, Compos. Sci. Technol. 2006, 66, 1198.

[210] M.N. Tahir, N. Zink, M. Eberhardt, H.A. Therese, U. Kolb, P. Theato, W. Tremel, Angew. Chem., Int. Ed. 2006, 45, 4809.

[211] S. Meuer, P. Oberle, P. Theato, W. Tremel, R. Zentel, Adv. Mater. 2007, 19, 2073.

[212] S. Meuer, K. Fischer, I. Mey, A. Janshoff, M. Schmidt, R. Zentel, Macromolecules 2008, 41, 7946.

[213] M. Zorn, M.N. Tahir, B. Bergmann, W. Tremel, C. Grigoriadis, G. Floudas, R. Zentel, Macromol. Rapid Commun. 2010, 31, 1101.

[214] L. de la Garza, Z.V. Saponjic, N.M. Dimitrijevic, M.C. Thurnauer, T. Rajh, J. Phys. Chem. B 2006, 110, 680.

[215] L. de la Garza, Z.V. Saponjic, T. Rajh, N.M. Dimitrijevic, Chem. Mater. 2006, 18, 2682.

[216] EP0501227 B1

[217] X.D. Pan, Z. Qin, Y.Y. Yan, P. Sadhukhan, Polymer 2010, 51, 3453. 
[218] L. Yang, S.L. Phua, J.K.H. Teo, C.L. Toh, S. K. Lau, J. Ma, X. Lu, ACS Appl. Mater. Interfaces 2011, 3, 3026.

[219] L. Zhu, Y. Lu, Y. Wang, L. Zhang, W. Wang, Appl. Surf. Sci. 2012, 258, 5387.

[220] D. Hirsemann, S. Shylesh, R. A. de Souza, B. Diar-Bakerly, B. Biersack, D. N. Mueller, M. Martin, R. Schobert, J. Breu, Angew. Chem. Int. Ed. 2012, 51, 1348.

[221] A. Bourmaud, J. Riviere, A. Le Duigou, G. Raj, C. Baley, Polymer Test. 2009, 28, 668.

[222] S. H. Yang, E. H. Ko, I. S. Choi, Langmuir 2012, 28, 2151.

[223] J. Xia, J. Lin, Y. Xu, Q. Chen, ACS Appl. Mater. Interfaces 2011, 3, 482.

[224] S. Kobayashi, H. Uyama, R. Ikeda, Chem. Eur. J. 2001, 7, 4755.

[225] J. Kumanotani, Prog. Org. Coat. 1998, 34, 135.

[226] a) K. H. Markiewitz, C. R. Dawson, J. Org. Chem. 1965, 30, 1610. b) L. A. Walker, E. S. Watson, M. A. EISohly, Immunopharmacol. Immunotoxicol. 1995, 17, 565.

[227] a) O. Vogl, J. Polym. Sci. Part A: Polym. Chem. 2000, 38, 4327. b) N. Niimura, Int. J. Mass. Spectrom. 2009, 284, 93.

[228] a) J. Kumanotani, Prog. Org. Coat. 1995, 26, 163. b) R. Oshima, Y. Yamauchi, C. Watanabe, J. Kumanotani, J. Org. Chem. 1985, 50, 2613.

[229] D. E. Bergbreiter, K-S Liao, Soft Matter 2009, 5, 23.

[230] R. Lu, S. Harigaya, T. Ishimura, K. Nagase, T. Miyakoshi, Prog. Org. Coat. 2004, 51, 238.

[231] a) T. Ishimura, R. Lu, K. Yamasaki, T. Miyakoshi, Prog. Org. Coat. 2008, 62, 193. b) T. Ishimura, R. Lu, K. Yamasaki, T. Miyakoshi, Prog. Org. Coat. 2010, 69, 12.

[232] J. Xia, Y. Xu, J. Lin, B. Hu, Prog. Org. Coat. 2008, 61, 7.

[233] J. Xia, Y. Xu, J. Lin, Prog. Org. Coat. 2010, 67, 365.

[234] J. Xia, Y. Xu, B. Hu, J. Lin, Prog. Org. Coat. 2009, 65, 510.

[235] Y. Liu, J. Xia, J. Lin, Prog. Org. Coat. 2011, 71, 117.

[236] J. Xia, Y. Xu, J. Lin, B. Hu, Prog. Org. Coat. 2009, 63, 1499.

[237] Y. Xu, J. Xia, B. Hu, Prog. Org. Coat. 2009, 65, 25.

[238] J. Xia, J. Lin, Y. Xu, Chem. Lett. 2010, 39, 1130.

[239] W. Bai, Y. Xu, Z. Tong, J. Lin, Optoelectron. Adv. Mat. 2011, 5, 22.

[240] H. S. Kim, J. H. Yeum, S. W. Choi, J. Y. Lee, I. W. Cheong, Prog. Org. Coat. 2009, 65, 341.

[241] X.-L. Zheng, J.-B. Weng, Q.-M. Huang, B.-H. Hu, T. Qiao, P. Deng, Colloid Surf. A 2009, 337, 15. 
[242] X.-L. Zheng, J.-B. Weng, B.-H. Hu, X.-Z. Lv, D. L. Deng, A. S. C. Chan, Mater. Chem. Phys. 2011, 130, 1054.

[243] T. Tsujimoto, N. Ando, H. Oyabu, H. Uyama, S. Kobayashi, J. Macromol. Sci. Pure 2007, 44, 1055.

[244] T. Tsujimoto, R. Ikeda, H. Uyama, S. Kobayashi, Chem. Lett. 2000, 29, 1122.

[245] H. Xu, Z. Lu, G. Zhang, RSC Advances 2012, 2, 2768.

[246] G. Prota, J. Invest. Dermatol. 1980, 75, 122.

[247] P. Meredith, T. Sarna, Pigment Cell Res. 2006, 19, 572.

[248] J. D. Simon, D. N. Pelles, Accounts Chem. Res. 2010, 43, 1452.

[249] H. S. Raper, J. Biochem. 1927, 21, 89.

[250] H. S. Mason, J. Biol. Chem. 1947, 168, 433.

[251] S. Ito, Pigment Cell Res. 2003, 16, 230.

[252] A. A. R. Watt, J. P. Bothma, P. Meredith, Soft Matter 2009, 5, 3754.

[253] M. D. Ischia, A. Napolitano, A. Pezzella, P. meredith, T. Sarna, Angew. Chem., Int. Ed. 2009, 48, 3914.

[254] V. Liu, J. D. Simon, Pigment Cell Res. 2003, 16, 606.

[255] M. Rozanowska, T. Sarna, E. J. Land, T. G. Truscott, Free Radical Bio. Med. 1999, 26, 518.

[256] J. B. Nofsinger, T. Ye, J. D. Simon, J. Phys. Chem. B 2001, 105, 2864.

[257] P. Meredith, J. Riesz, Photochem. Photobiol. 2004, 79, 211.

[258] J. McGinness, P. Corry, P. Proctor, Science 1974, 183, 853.

[259] C. M. R. Clancy, J. D. Simon, Biochemistry 2001, 40, 13353.

[260] P. Meredith, B. J. Powell, J. Riesz, S. P. Nighswander-Rempel, M. R. Pederson, E. G. Moore, Soft Matter 2006, 2, 37.

[261] E. Kaxiras, A. Tsolakidis, G. Zonios, S. Meng, Phys. Rev. Lett. 2006, 97, 218102.

[262] S. Meng, E. Kaxiras, Biophys. J. 2008, 95, 4396.

[263] W. L. Cheun, Pigment Cell Res. 2004, 17, 422.

[264] S. Meng, E. Kaxiras, Biophys. J. 2008, 94, 2095.

[265] A. Pizzella, A. ladosini, S. Valerio, L. Pancella, A. Napolitano, M. Adinolfi, M. D. Ischia, J. Am. Chem. Soc. 2009, 131, 15270.

[266] W. D. Bush, J. Garguilo, F. A. Zucca, A. Albertini, L. Zecca, G. S. Edwards, R. J. Nemanich, J. D. Simon, Proc.Natl. Acad. Sci. USA. 2006, 103, 14785. 
[267] K.-Y. Ju, Y. Lee, S. Lee, S. B. Park, J.-K. Lee, Biomacromolecules 2011, 12, 625.

[268] F. Bernsmann, A. Ponche, C. Ringwald, J. Hemmerlé, J. Raya, B. Bechinger, J.-C. Voegel, P. Schaaf, V. Ball, J. Phys. Chem. C 2009, 113, 8234.

[269] J. Ryu, S. H. Ku, M. Lee, C. B. Park, Soft Matter 2011, 7, 7201.

[270] C. C. Félix, J. S. Hyde, T. Sarna, R. C. Sealy, J. Am. Chem. Soc. 1978, 100, 3922.

[271] G. M. Gadd, Mycol. Res. 2007, 111, 3.

[272] R. V. Fogarty, J. M. Tobin, Enzyme Microb. Tech. 1996, 19, 311.

[273] K. L. Double, L. Zecca, P. Costi, M. Mauer, C. Griesinger, S. Ito, D. Ben-Shachar, G. Bringmann, R. G. Fariello, P. Riederer, M. Gerlach, J. Neurochem. 2000, 75, 2583.

[274] M. D. Ischia, G. Prota, Pigment Cell Res. 1997, 10, 370.

[275] L. Hong, J. D. SImon, J. Phys. Chem. B 2007, 111, 7938.

[276] K. J. Lawrie, P. Meredith, R. P. McGeary Photochem. Photobiol. 2008, 84, 632.

[277] S. Subianto, G. Will, P. Meredith, Polymer, 2005, 46, 11505.

[278] J. E. D. Albuquerque, C. Giacomantonio, A. G. White, P. Meredith, Eur. Biophys. J. 2006, 35, 190.

[279] J. P. Bothma, J. D. Boor, U. Divakar, P. E. Schwenn, P. Meredith, Adv. Mater. 2008, 20, 3539.

[280] A. G. Orive, P. Dip, Y. Gimeno, P. Díaz, P. Carro, A. H. Creus, G. Benítez, P. L. Schilardi, L. Andrini, F. Requejo, R. C. Salvarezza, Chem. Eur. J. 2007, 13, 473.

[281] M. Ambrico, P. F. Ambrico, A. Cardone, T. Ligonzo, S. R. Cicco, R. D. Mundo, V. Augelli, G. M. Farinola, Adv. Mater. 2011, 23, 3332.

[282] Q. Zhao, X. Guo, T. Fan, J. Ding, D. Zhang, Q. Guo, Soft Matter 2011, 7, 11433.

[283] K. Shanmuganathan, J. H. Cho, P. Iyer, S. Baranowitz, C. J. Ellison, Macromolecules 2011, 44, 9499.

[284] B. Yu, D. A. Wang, Q. Ye, F. Zhou, W. Liu, Chem. Commun. 2009, 6789.

[285] J. Cui, Y. Wang, A. Postma, J. Hao, L. Hosta-Rigau, F. Caruso, Adv. Funct. Mater. 2010, 20, 1625.

[286] Q. Liu, B. Yu, W. Ye, F. Zhou, Macromol. Biosci. 2011, 11, 1227.

[287] L. Zhang, J. Shi, Z. Jiang, Y. Jiang, S. Qiao, J. Li, R. Wang, R. Meng, Y. Zhu, Y. Zheng, Green Chem. 2011, 13, 300.

[288] L. Zhang, J. Shi, Z. Jiang, Y. Jiang, R. Meng, Y. Zhu, Y. Liang, Y. Zheng, ACS Appl. Mater. Interfaces 2011, 3, 597.

[289] E. P. Holowka, T. J. Deming, Macromol. Biosci. 2010, 10, 496. 
[290] M. N. Tahir, P. Theato, P. Oberle, G. Melnyk, S. Faiss, U. Kolb, A. Janshoff, M. Stepputat, W. Tremel, Langmuir 2006, 22, 5209.

[291] S. Kim, C. B. Park, Biomaterials 2010, 31, 6628.

[292] S. Kim, C. B. Park, Langmuir 2010, 26, 14730.

[293] K. C. L. Black, Z. Liu, P. B. Messersmith, Chem. Mater. 2011, 23, 1130.

[294] J. Ryu, S. H. Ku, H. Lee, C. B. Park, Adv. Funct. Mater. 2010, 20, 2132.

[295] M. Lee, S. H. Ku, J. Ryu, C. B. Park, J. Mater. Chem. 2010, 20, 8848.

[296] C. Wu, W. Fan, J. Chang, Y. Xiao, J. Mater. Chem. 2011, 21, 18300.

[297] D. L. Caulder, K. N. Raymond, J. Chem. Soc. Dalton Trans. 1999, 1185.

[298] D. L. Caulder, R. E. Powers, T. N. Parac, K. N. Raymond, Angew. Chem. Int. Ed. 1998, 37, 1840.

[299] D. Fiedler, R. G. Bergman, K. N. Raymond, Angew. Chem. Int. Ed. 2004, 43, 6748.

[300] D. Fiedler, H. V. Halbeek, R. G. Bergman, K. N. Raymond, J. Am. Chem. Soc. 2006, 128, 10240.

[301] C. J. Hastings, D. Fiedler, R. G. Bergman, K. N. Raymond, J. Am. Chem. Soc. 2008, 130, 10977.

[302] D. H. Leung, D. Fiedler, R. G. Bergman, K. N. Raymond, Angew. Chem. Int. Ed. 2004, 43, 963.

[303] D. H. Leung, R. G. Bergman, K. N. Raymond, J. Am. Chem. Soc. 2006, 128, 9781.

[304] D. H. Leung, R. G. Bergman, K. N. Raymond, J. Am. Chem. Soc. 2007, 129, 2746.

[305] J. L. Brumaghim, M. Michels, D. Pagliero, K. N. Raymond, Eur. J. Org. Chem. 2004, 5115.

[306] V. M. Dong, D. Fiedler, B. Carl, R. G. Bergman, K. N. Raymond, J. Am. Chem. Soc. 2006, 128, 14464.

[307] D. Fiedler, R. G. Bergman, K. N. Raymond, Angew. Chem. Int. Ed. 2006, 45, 745.

[308] M. D. Pluth, R. G. Bergman, K. N. Raymond, J. Am. Chem. Soc. 2007, 129, 11459.

[309] M. D. Pluth, R. G. Bergman, K. N. Raymond, Angew. Chem. Int. Ed. 2007, 46, 8587.

[310] M. D. Pluth, R. G. Bergman, K. N. Raymond, Accounts Chem. Res. 2009, 42, 1650.

[311] M. D. Pluth, R. G. Bergman, K. N. Raymond, J. Am. Chem. Soc. 2008, 130, 11423.

[312] C. E. Lankford, B. R. Byers, Crit. Rev. Microbiol. 1973, 2, 273.

[313] M. Sandy, A. Butler, Chem. Rev. 2009, 109, 4580.

[314] R. C. Hider, X. Kong, Nat. Prod. Rep. 2010, 27, 637.

[315] M. Miethke, M. A. Marahiel, Microbiol. Mol. Biol. R. 2007, 71, 413.

[316] K. N. Raymond, E. A. Dertz, S. S. Kim, Proc. Natl. Acad. Sci. USA. 2003, 100, 3584.

[317] K. Konopka, J. B. Neilands, Biochemistry 1984, 23, 2122. 
[318] G. Bao, M. Clifton, T. M. Hoette, K. Mori, S.-X. Deng, A. Qiu, M. Viltard, D. Williams, N. Paragas, T. Leete, R. Kulkarni, X. Li, B. Lee, A. Kalandadze, A. J. Ratner, J. C. Pizarro, K. M. Schmidt-Ott, D. W. Landry, K. N. Raymond, R. K. Strong, J. Barasch, Nat. Chem. Biol. 2010, 6, 602.

[319] T. Ito, J. B. Neilands, J. Am. Chem. Soc. 1958, 80, 4645.

[320] R. J. Calugay, H. Takeyama, D. Mukoyama, Y. Fukuda, T. Suzuki, K. Kanoh, T. Matsunaga, J. Biosci. Bioeng. 2006, 101, 445.

[321] J. R. Pollack, J. B. Neilands, Biochem. Biophys. Res. Commun. 1970, 38, 989.

[322] J. J. May, T. M. Wendrich, M. A. Marahiel, J. Biol. Chem. 2001, 276, 7209.

[323] J. Y. Lee, K. D. Passalacqua, P. C. Hanna, D. H. Sherman, PLoS ONE 2011, 6, e20777.

[324] G. L. Griffiths, S. P. Sigel, S. M. Payne, J. B. Neilands, J. Biol. Chem. 1984, 259, 383.

[325] C. J. Carrano, K. N. Raymond, J. Am. Chem. Soc. 1979, 101, 5401.

[326] R. C. Scarrow, P. E. Riley, K. Abu-Dari, D. L. White, K. N. Raymond, Inorg. Chem. 1985, 24, 954.

[327] C. M. Peterson, J. H. Graziano, R. W. Grady, Br. J. Haematol. 1976, 33, 447.

[328] C. G. Pitt, G. Gupta, W. E: Estes, H. Rosenkrantz, J J. Metterville, A. L. Crumbliss, R. A. Palmer, K. W. Nordquest, K. A. S. Hardy, D. R. Whitcomb, B. R. Byers, J. E. L. Arceneaux, C. G. Gaines, C. V. Sciortino, J. Pharmacol. Exp. Ther. 1979, 208, 12.

[329] R. C. Hider, G. Kontoghiorghes, J. Silver, UK Patent Application 2118 176, 1983.

[330] G. Kontoghiorghes, Biochem. J. 1986, 233, 299.

[331] A. M. Hardemare, S. torelli, G. Serratrice, J.-L. Pierre, Biometals 2006, 19, 349.

[332] F. L. Weitl, K. N. Raymond, J. Am. Chem. Soc. 1979, 101, 2728.

[333] W. R. Harris, F. L. Weitl, K. N. Raymond, J. Chem. Soc. Chem. Comm. 1979, 177.

[334] D. L. Caulder, K. N. Raymond, Acc. Chem. Res. 1999, 32, 975.

[335] R. Schobert, A. Stangl, K. Hannemann, Tetrahedron 2008, 64, 1711.

[336] K. A. Mies, P. Gebhardt, U. Möllmann, A. L. Crumbliss, J. Inorg. Biochem. 2008, 102, 850.

[337] R. J. Abergel, K. N. Raymond, Inorg. Chem. 2006, 45, 3622.

[338] R. C. Scarrow, D. L. White, K. N. Raymond, J. Am. Chem. Soc. 1985, 107, 6540.

[339] J. Xu, B. O’Sullivan, K. N. Raymond, Inorg. Chem. 2002, 41, 6731.

[340] R. A. Yokel, A. M. Fredenburg, P. W. Durbin, J. Xu, M. K. Rayens, K. N. Raymond, J. Pharm. Sci. 2000, 89, 545.

[341] Y. Bethuel, K. Gademann, J. Org. Chem. 2005, 70, 6258. 
[342] M. Apostol, P. Baret, G. Serratrice, J. Desbrières, J.-L. Putaux, M.-J. Stebe, D. Expert, J.-L. Pierre, Angew. Chem. Int. Ed. 2005, 44, 2580.

[343] L. Bednarova, J. Brandel, A. M. Hardemare, J. Bednar, G. Serratrice, J.-L. Pierre, Chem. Eur. J. 2008, 14,3680 .

[344] R. Yadav, R. Kikkeri, Chem. Commun. 2012, 48, 1704.

[345] L. D. Loomis, K. N. Raymond, Inorg. Chem. 1991, 30, 906.

[346] a) T. B. Karpishin, T. M. Dewey, K. N. Raymond, J. Am. Chem. Soc. 1993, 115, 1842. b)T. B. Karpishin, K. N. Raymond, Angew. Chem. Int. Ed. 1992, 31, 466.

[347] P. Cornelis J. Bacteriol. 2008, 190, 6541.

[348] E. Wasielewski, R. A. Atkinson, M. A. Abdallah, B. Kieffer, Biochemistry 2002, 41, 12488.

[349] M. Bouby, I. Millard, J. MacCordick, I. Rossini, Radiochim. Acta 1998, 80, 95.

[350] A. K. Duhme, R. C. Hider, M. J. Naldrett, R. N. Pau, J. Biol. Inorg. Chem. 1998, 3, 520.

[351] H. H. Khodr, R. C. Hider, A.-K. Duhme-Klair, J. Biol. Inorg. Chem. 2002, 7, 891.

[352] A.-K. Duhme-Klair, Eur. J. Inorg. Chem. 2009, 3689.

[353] F. L. Weitl, K. N. Raymond, J. Am. Chem. Soc. 1980, 102, 2289.

[354] F. L. Weitl, K. N. Raymond, US Patent 4442 305, 1984.

[355] F. L. Weitl, K. N. Raymond, US Patent 4309 305, 1982.

[356] A. E. V. Gorden, J. Xu, K. N. Raymond, Chem. Rev. 2003, 103, 4207.

[357] R. J. Abergel, K. N. Raymond, Hemoglobin 2011, 35, 276.

[358] Y. Miao, T. P. Quinn, Crit. Rev. Oncol. Hemat. 2008, 67, 213.

[359] J. Su, F. Chen, V. L. Cryns, P. B. Messersmith, J. Am. Chem. Soc. 2011, 133, 11850.

[360 V. Braun, A. Pramanik, T. Gwinner, M. Köberle, E. Bohn, Biometals 2009, 22, 3.

[361 S. Duquesne, D. Destoumieux-Garzón, J. Peduzzi, S. Rebuffat, Nat. Prod. Rep. 2007, 24, 708.

[362] M. F. Azpiroz, M. Laviña, Antimicrob. Agents Chemother. 2004, 48, 1235.

[363] X. Thomas, D. Destoumieux-Garzón, J. Peduzzi, C. Alfonso, A. Blond, N. Birlirakis, C. Goulard, L. Dubost, R. Thai, J.-C. Tabet, S. Rebuffat, J. Biol. Chem. 2004, 279, 28233.

[364] D. Destoumieux-Garzón, J. Peduzzi, X. Thomas, C. Djediat, S. Rebuffat, Biometals 2006, 19, 181.

[365] S. I. Patzer, M. R. Baquero, D. Bravo, F. Moreno, K. Hantke, Microbiology 2003, 149, 2557.

[366] M. J. Miller, F. Malouin, Acc. Chem. Res. 1993, 26, 241.

[367] H. Budzikiewicz, Curr. Top. Med. Chem. 2001, 1, 73. 
[368] V. Braun, Drug Resist. Update. 1999, 2, 363.

[369] M. J. Miller, H. Zhu, Y. Xu, C. Wu, A. J. Walz, A. Vergne, J. M. Roosenberg, G. Moraski, A. A. Minnick, J. McKee-Dolence, J. Hu, K. Fennell, E. K. Dolence, L. Dong, S. Franzblau, F. Malouin, U. Möllmann, Biometals 2009, 22, 61.

[370] H. Heli, S. Mirtorabi, K. Karimian, Expert Opin. Ther. Patents 2011, 21, 819.

[371] N. A. Watanabe, T. Nagasu, K. Katsu, K. Kitoh, Antimicrob. Agents Chemother. 1987, 31, 497.

[372] J. D. Buynak, Curr. Med. Chem. 2004, 11, 1951.

[373] M. Ghosh, M. J. Miller, Bioorgan. Med. Chem. 1996, 4, 43.

[374] A. Ghosh, M. Ghosh, C. Niu, F. Malouin, U. Moellmann, M. J. Miller, Chem. Biol. 1996, 3, 1011.

[375] O. Kinzel, R. Tappe, I. Gerus, H. Budzikiewicz, J. Antibiot. 1998, 51, 499.

[376] K. A. Mies, P. Gebhardt, U. Möllmann, A. L. Crumbliss, J. Inorg. Biochem. 2008, 102, 850.

[377] J. Fung-Tomc, K. Bush, B. Minassian, B. Kolek, R. Flamm, E. Gradelski, D. Bonner, Antimicrob. Agent. Chemother. 1997, 41, 1010.

[378] K. Obi, A. Kojima, H. Fukuda, K. Hirai, Bioorgan. Med. Chem. Lett. 1995, 5, 2777.

[379] T. A. Russo, M. G. P. Page, J. M. Beanan, R. Olson, A. M. Hujer, K. M. Hujer, M. Jacobs, S. Bajaksouzian, A. Endimiani, R. A. Bonomo, J. Antimicrob. Chemother. 2011, 66, 867.

[380] C. Hennard, Q. C. Truong, J.-F- Desnottes, J.-M. Paris, N. J. Moreau, M. A. Abdallah, J. Med. Chem. 2001, 44, 2139.

[381] L. Heinisch, S. Wittmann, T. Stoiber, A. Berg, D. Ankel-Fuchs, U. Möllmann, J. Med. Chem. 2002, 45, 3032.

[382 L. Heinisch, S. Wittmann, I. Scherlitz-Hofmann, T. Stoiber, A. Berg, U. Möllmann, US Patent Application 0132707 A1, 2004.

[383] L. Heinisch, S. Wittmann, T. Stoiber, I. Scherlitz-Hofmann, D. Ankel-Fuchs, U. Mollmann, Arzneimittel Forsch. 2003, 53, 188.

[384] U. Möllmann, L. Heinisch, A. Bauernfeind, T. Köhler, D. Ankel-Fuchs, Biometals 2009, 22, 615

[385] S. Grafe, P. Gebhardt, V. Albrecht, US Patent Application 0186087 A1, 2004.

[386] O. Hiroyuki, I. Masashi, Y. Yoshiyuki, E. Isamu, U. Hideto, JP Patent Application 2017146 A1, 1990.

[387] A. Abbruzzese, H. M. Hanauskeabel, M. H. Park, S. Henke, J. E: Folk, Biochim. Biophys. Acta 1991, 1077, 159.

[388] H. M. Hanauske-abel, WO Patent Application 92/05190 A1, 1990. 
[389] E. J. Werner, A. Datta, C. J. Jocher, K. N. Raymond, Angew. Chem. Int. Ed. 2008, 47, 8568.

[390] A. Datta, K. N. Raymond, Acc. Chem. Res. 2009, 42, 938.

[391] P. Caravan, J. J. Ellison, T. J. McMurry, R. B. Lauffer, Chem. Rev. 1999, 99, 2293.

[392] J. Xu, S. J. Franklin, D. W. Whisenhunt, K. N. Raymond, J. Am. Chem. Soc. 1995, 117, 7245.

[393] S. M. Cohen, J. Xu, E. Radkov, K. N. Raymond, M. Botta, A. Barge, S. Aime, Inorg. Chem. 2000, 39, 5747.

[394] D. M. J. Doble, M. Melchior, B. O’Sullivan, C. Siering, J. Xu, V. C. Pierre, K. N. Raymond, Inorg. Chem. 2003, 42, 4930.

[395] K. N. Raymond, V. C. Pierre, Bioconjugate Chem. 2005, 16, 3.

[396] M. K. Thompson, B. Misselwitz, L. S. Tso, D. M. J. Doble, H. Schmitt-Willich, K. N. Raymond, J. Med. Chem. 2005, 48, 3874.

[397] W. C. Floyd, P. J. Klemm, D. E. Smiles, A. C. Kohlgruber, V. C. Pierre, J. L. Mynar, J. M. J. Fréchet, K. N. Raymond, J. Am. Chem. Soc. 2011, 133, 2390.

[398] J. M. Hooker, A. Datta, M. Botta, K. N. Raymond, M. B. Francis, Nano Lett. 2007, 7, 2207.

[399] G. Dehaen, S. V. Eliseeva, K. Kimpe, S. Laurent, L. V. Elst, R. N. Muller, W. Dehaen, K. Binnemans, T. N. Parac-Vogt, Chem. Eur. J. 2012, 18, 293.

[400] E. G. Moore, A. P. S. Samuel, K. N. Raymond, Acc. Chem. Res. 2009, 42, 542.

[401] C. Tedeschi, J. Azéma, H. Goritzka, P. Tisnès, C. Picard, Dalton Trans. 2003, 1738.

[402] E. G. Moore, J. Xu, C. J. Jocher, E. J. Werner, K. N. Raymond, J. Am. Chem. Soc. 2006, 128, 10648.

[403] J. D. Buynak, A. S. Rao, V. R. Doppalapudi, G. Adam, P. J. Petersen, S. D. Nidamarthy, Bioorg. Med. Chem. Lett. 1999, 9, 1997.

[404] E. G. Moore, J. Xu, C. J. Jocher, I. Castro-Rodriguez, K. N. Raymond, Inorg. Chem. 2008, 47, 3105.

[405] E. G. Moore, J. Xu, S. C. Dodani, C. J. Jocher, A. D’Aleo, M. Seitz, K. N. Raymond, Inorg. Chem. 2010, $49,4156$.

[406] P. C. Nagajyoti, K. D. Lee, T. V. M. Sreekanth, Environ. Chem. Lett. 2010, 8, 199.

[407] K. E. Giller, E. Witter, S. P. McGrath, Soil Biol. Biochem. 1998, 30, 1389.

[408] M. W. H. Evangelou, M. Ebel, A. Schaeffer, Chemosphere, 2007, 68, 989.

[409] M. Halim, P. Conte, A. Piccolo, Chemosphere, 2003, 52, 265.

[410] A. A. Belimov, N. Hontzeas, V. I. Safronova, S. V. Demchinskaya, G. Piluzza, S. Bullitta, B. R. Glick, Soil. Biol. Biochem. 2005, 37, 241. 
[411] T. Lebeau, A. Braud, K. Jezequel, Environ. Pollut. 2008, 153, 497.

[412] A. Braud, K. Jezequel, S. Bazot, T. Lebeau, Chemosphere 2009, 74, 280.

[413] D. H. Ellis, D. A. Griffiths, Can. J. Microbiol. 1974, 20, 1379.

[414] P. Baldrian, Enzyme Microb. Technol. 2003, 32, 78.

[415] D. M. Rizzo, R. A. Blanchette, M. A. Palmer, Can. J. Bot. 1992, 70, 1515.

[416] P. M. Shannon, V. Unnithan, S. Bouriak, P. Chachkine, Nature 1998, 391, 649.

[417] M. A. Fomina, V. M. Kadoshnikov, B. P. Zlobenko, in Process Metallurgy, Vol. 9 (Eds: R. Amils, A. Ballester), UCM-UAM, Madrid, Spain 1999, 245.

[418] P. Burba, M. Griesbach, K. H. Lieser, Fresenius J. Anal. Chem. 1977, 284, 257.

[419] M. Griesbach, L, H. Lieser, K. H. Lieser, Fresenius J. Anal. Chem. 1980, 302, 109.

[420] S. Iwabuchi, T. Nakahira, Y. Fukushima, O. Saito, K. Kojima, J. Pol. Sci.- Pol. Chem. Ed. 1981, $19,785$.

[421] A. N. Pustam, S. D. Alexandratos, React. Funct. Polym. 2010, 70, 545.

[422] S.-P. Huang, W. Li, K. J. Franz, R. L. Albright, R. H. Fish, Inorg. Chem. 1995, 34, 2813.

[423] R. H. Fish, US Patent 5834 525, 1998.

[424] S.-P. Huang, K. J. Franz, M. M. Olmstead, R. H. Fish, Inorg. Chem. 1995, 34, 2820.

[425 R. H. Fish, US Patent 4552 854, 1985.

[426] R. H. Fish, US Patent 4604 191, 1986.

[427] J. L. Seela, K. Folting, R.J. Wang, J. C. Huffman, G. Christou, H.-R. Chang, D. N. Hendrickson, Inorg. Chem. 1985, 24, 4456.

[428] M. J. Sever, J. J. Wilker, Dalton Trans. 2004, 1061.

[429] P. K. Tewari, A. K. Singh, Talanta 2001, 53, 823.

[430] M. Kumar, D. P. S. Rathore, A. K. Singh, Analyst. 2000, 125, 1221.

[431] V. A. Lemos, D. G. da Silva, A. L. de Carvalho, D. D. A. Santana, G. D. S. Novaes, A. S. dos Passos, Microchem. J. 2006, 84, 14.

[432] D. Kara, A. Fisher, S. J. Hill, Analyst, 2006, 131, 1232.

[433] G. Venkatesh, A. K. Singh, Talanta 2005, 67, 187.

[434] D. Prabhakaran, M. S. Subramanian, Talanta 2003, 61, 431.

[435] G. Venkatesh, A. K. Singh, Talanta 2007, 71, 282.

[436] I. Narin, M. Tuzen, M. Soylak, Talanta 2004, 63, 411. 
[437] V. A. Lemos, A. S. dos Passos, G. D. S. Novaes, D. D. A. Santana, A. L. de Carvalho, D. G. da Silva, React. Funct. Polym. 2007, 67, 573.

[438] L. Li, Y. Li, X. Luo, J. Deng, W. Yang, React. Funct. Polym. 2010, 70, 938.

[439] G. Venkatesh, A. K. Singh, B. Venkataramani, Microchim. Acta 2004, 114, 233.

[440] V. Gurnani, A. K. Singh, B. Venkataramani, Talanta 2003, 61, 889.

[441] J. Bernard, C. Branger, T. L. A. Nguyen, R. Denoyel, A. Margaillan, React. Funct. Polym. 2008, 68, 1362.

[442] D. Prabhakaran, M. S. Subramanian, Talanta 2003, 61, 423.

[443] A. Sabarudin, M. Oshima, T. Takayanagi, L. Hakim, K. Oshita, Y. H. Giao, S. Motomizu, Anal. Chim. Acta 2007, 581, 214.

[444] A. Sabarudin, O. Noguchi, M. Oshima, K. Higuchi, S. Motomizu, Microchim. Acta 2007, $159,341$.

[445] J. Bernard, C. Branger, I. Beurroies, R. Denoyel, S. Blanc, A. Margaillan, Polymer 2010, 51, 2472.

[446] H. Deleuze, X. Schulze, D. C. Sherrington, J. Polym. Sci. Pol. Chem. 2000, 38, 2879.

[447] M. Mure, Acc. Chem. Res. 2004, 37, 131.

[448] C. Anthony, Antiox. Redox. Signal 2001, 3, 757.

[449] S. X. Wang, M. Mure, K. F. Medzihradszky, A. L. Burlingame, D. E. Brown, D. M. Dooley, A. J. Smith, H. M. Kagan, J. P. Klinman, Science 1996, 273, 1078.

[450] H. M. Kagan, P. C. Trackman, Am. J. Respir. Cell Mol. Biol. 1991, 5, 206.

[451] C. Anthony, Biochem. J. 1996, 320, 697.

[452] T. Kasahara, T. Kato, Nature 2003, 422, 832.

[453] L. M. Felton, C. Anthony, Nature 2005, 433, E10.

[454] T. Kasahara, T. Kato, Nature 2005, 433, E11.

[455] J. Moiroux, P. J. Elving, J. Am. Chem. Soc. 1980, 102, 6533.

[456] W. J. Blaedel R. A. Jenkins, Anal. Chem. 1975, 47, 1337.

[457] D. C.-S. Tse, T. Kuwana, Anal. Chem. 1978, 50, 1315.

[458] B. W. Carlson, L. L. Miller, J. Am. Chem. Soc. 1985, 107, 479.

[459] P. N. Barlett, E. Simon, C. S. Toh, Bioelectrochemistry. 2002, 56, 117.

[460] H. Jaegfeldt, A. B. C: Torstensson, L. G. O. Gorton, G. Johansson, Anal. Chem. 1981, 53, 1979.

[461] H. Jaegfeldt, T. Kuwana, G. Johansson, J. Am. Chem. Soc. 1983, 105, 1805.

[462] E. Lorenzo, L. Sánchez, F. Pariente, J. Tirado, J. D. Abruña, Anal. Chim. Acta 1995, 309, 79. 
[463] J.-J. Sun, J.-J. Xu, H.-Q. Fang, H.-Y. Chen, Bioelectrochem. Bioenerg. 1997, 44, 45.

[464] J. Kang, L. Zhuo, X. Lu, X. Wang, J. Solid State Electrochem. 2005, 9, 114.

[465] F. Pariente, F. Tobalina, M. Darder, E. Lorenzo, H. D. Abruña, Anal. Chem. 1996, 68, 3135.

[466] C. A. Goss, H. D. Abruña, Inorg. Chem. 1985, 24, 4263.

[467] Z. Wu, W. Jing, E. Wang, Electrochem. Commun. 1999, 1, 545.

[468] X. Han, J. Tang, J. Wang, E. Wang, Electrochim. Acta 2001, 46, 3367.

[469] D. J. Weiss, M. Dorris, A. Loh, L. Peterson, Biosens. Bioelectron. 2007, 22, 2436.

[470] C. Degrand, L. L. Miller, J. Am. Chem. Soc. 1980, 102, 5728.

[471] J. Davis, D. H. Vaughan, M. F. Cardosi, Electrochim. Acta 1997, 43, 291.

[472] G. Qian, C. Yang, W. Pu, J. Huang, J. Zhang, Synthetic. Met. 2007, 157, 448.

[473] J. Bai, X. Bo, B. Qi, L. Guo, Electroanalysis 2010, 22, 1750.

[474] A. S. Kumar, P. Swetha, Langmuir 2010, 26, 6874.

[475] E. Lorenzo, F. Pariente, L. Hernandez, F. Tobalina, M. Darder, Q. Wu, M. Maskus, H. D. Abruña, Biosens. Bioelectron. 1998, 13, 319.

[476] C. Ueda, D. C.-S. Tse, T. Kuwana, Anal. Chem. 1982, 54, 850.

[477] I. M. Alfaro, E. I. Pizarro, L. Rodríguez, E. M. Valdés, Bioelectrochem. Bioenerg. 1995, 38, 307.

[478] P.-Y. Chen, P.-C. Nien, C.-T. Wu, T.-H. Wu, C.-W. Lin, K.-C. Ho, Anal. Chim. Acta 2009, 643, 38.

[479] M. D. Rubianes, G. A. Rivas, Anal. Chim. Acta 2001, 440, 99.

[480] C. R. Raj, S. Chakraborty, Biosens. Bioelectron. 2006, 22, 700.

[481] H. R. Zare, S. M. Golabi, J. Electroanal. Chem. 1999, 464, 14.

[482] H. R. Zare, S. M. Golabi, J. Solid State Electrochem. 2000, 4, 87.

[483] A. Ciszewski, G. Milczarek, Anal. Chem. 2000, 72, 3203.

[484] S. M. Golabi, H. R. Zare, M. Hamzehloo, Electroanalysis 2002, 14, 611.

[485] G. Milezarek, Langmuir 2009, 25, 10345.

[486] J. Tang, Z. Wu, J. Wang, E. Wang, Electroanalysis 2001, 13, 1315.

[487] P.-Y. Chen, R. Vittal, P.-C. Nien, K.-C. Ho, Biosens. Bioelectron. 2009, 24, 3504.

[488] M. A. Ghanem, J.-M. Chrétien, J. D. Kilburn, P. N. Barlett, Biochemistry 2009, 76, 115.

[489] A. A. Ensafi, H. Karimi-Maleh, S. Mallakpour, B. Rezaei, Coll. Surf. B 2011, 87, 480.

[490] B. Ge, Y. Tan, Q. Xie, M. Ma, S. Yao, Sensor. Actuat. A-Chem. 2009, 137, 547.

[491] M. Wooten, W. Gorski, Anal. Chem. 2010, 82, 1299. 
[492] M. Fukui, A. Chantal, C. Degrand, L. L. Miller, J. Am. Chem. Soc. 1982, 104, 28.

[493] A. N. K. Lau, L. L. Miller, J. Am. Chem. Soc. 1983, 105, 5271.

[494] F. Pariente, E. Lorenzo, H. D. Abruña, Anal. Chem. 1994, 66, 4337.

[495] F. Pariente, F. Tobalina, G. Moreno, L. Hernández, E. Lorenzo, H. D. Abruña, Anal. Chem. 1997, 69, 4065.

[496] P. C. White, N. S. Lawrence Y. C. Tsai, J. Davis, R. G. Compton, Mikrochim. Acta 2001, 137, 87.

[497] S. Itoh, H. Fukushima, M. Komatsu, Y. Ohshiro, Chem. Lett. 1992, 1583.

[498] M. V. del Pozo, C. Alonso, F. Pariente, E. Lorenzo, Anal. Chem. 2005, 77, 2550.

[499] Q. Wu, M. Maskus, F. Pariente F. Tobalina, V. M. Fernández, E. Lorenzo, H. D. Abruña, Anal. Chem. $1996,68,3688$.

[500] F. Tobalina, F. Pariente, L. Hernández, H. D. Abruña, E. Lorenzo, Anal. Chim. Acta 1999, 395, 17.

[501] M.-Y. Hong, J.-Y. Chang, H. C. Yoon, J.-S. Kim, Biosens. Bioelectron. 2002, 17, 13.

[502] C. G. Bauer, A. V. Eremenko, E. Ehrentreich-Förster, F. F. Bier, A. Makower, H. B. Halsall, W. R. Heineman, F. W. Scheller, Anal. Chem. 1996, 68, 2453.

[503] E. Katz, D. D. Schlereth, H. L. Schmidt, J. Electroanal. Chem. 1994, 367, 59.

[504] E. Katz, T. Lotzbeyer, D. D. Schlereth, W. Schuhmann, H. L. Schmidt, J. Electroanal. Chem. 1994, $373,189$.

[505] I. Willner, G. Arad, E. Katz, Bioelectrochemistry Bioenergetics 1998, 44, 209.

[506] A. Riklin, E. Katz, I. Willner, A. Socker, A. F. Bückmann, Nature 1995, 376, 672.

[507] I. Willner, Science 2002, 298, 2407.

[508] I. Willner, V. Heleg-Shabtai, R. Blonder, E. Katz, G. Tao, J. Am. Chem. Soc. 1996, 118, 10321.

[509] A. Bardea, E. Katz, A. F. Bückmann, I. Willner, J. Am. Chem. Soc. 1997, 119, 9114.

[510] M. Zayats, E. Katz, I. Willner, J. Am. Chem. Soc. 2002, 124, 2110.

[511] M. Zayats, E. Katz, I. Willner, J. Am. Chem. Soc. 2002, 124, 14724.

[512] Y. Xiao, F. Patolsky, E. Katz, J. F. Hainfeld, I. Willner, Science 2003, 299, 1877.

[513] M. Zayats, E. Katz, R. Baron, I. Willner, J. Am. Chem. Soc. 2005, 127, 12400.

[514] S. M. Golabi, H. R. Zare, M. Hamzehloo, Electroanalysis 2002, 14, 611.

[515] I. Willner, E. Katz, Angew. Chem. Int. Ed. 2003, 42, 4576.

[516] E. Katz, O. Lioubashevski, I. Willner, J. Am. Chem. Soc. 2005, 127, 3979. 
[517] E. Graf, M. Wais Hosseini, R. Ruppert, A. de Cian, J. Fischer, J. Chem. Soc. Chem. Commun. 1995, 1505.

[518] S. B. Jedner, R. James, R. N. Perutz, A.-K. Duhme-Klair, J. Chem. Soc. Dalton Trans. 2001, 2327.

[519] A. F. A. Peacock, J. D. Batey, C. Raendler, A. C. Whitwood, R. N. Perutz, A.-K. Duhme-Klair, Angew. Chem. Int. Ed. 2005, 44, 1712.

[520] I. Prévot-Halter, T. J. Smith, J. Weiss, J. Org. Chem. 1997, 62, 2186.

[521] I. Prevot-Halter, J. Weiss, New J. Chem. 1998, 869.

[522] D. K. Smith, Org. Biomol. Chem. 2003, 1, 3874.

[523] K. J. Winstanley, D. K. Smith, J. Org. Chem. 2007, 72, 2803.

[524] D. A. Jose, P. Kar, D. Koley, B. Ganguly, W. Thiel, H. N. Ghosh, A. Das, Inorg. Chem. 2007, 46, 5576.

[525] W.-T. Gong, K. Hiratani, T. Oba, S. Ito, J. Incl. Phenom. Macrocycl. Chem. 2008, 61, 179.

[526] V. K. Bhardwaj, M. S. Hundal, G. Hundal, Tetrahedron 2009, 65,8556.

[527] S. K. Berezin, J. T. Davis, J. Am. Chem. Soc. 2009, 131, 2458.

[528] E. Evangelio, J. Hernando, I. Imaz, G. Bardají, R. Alibés, F. Busqué, D. Ruiz-Molina, Chem. Eur. J. 2008, 14, 9754.

[529] E. Evangelio, J. Saiz-Poseu, D. Maspoch, K. Wurst, F. Busque, D. Ruiz-Molina, Eur. J. Inorg. Chem. 2008, 2278.

[530] A. Martínez-Otero, E. Evangelio, R. Alibés, J. L. Bourdelande, D. Ruiz-Molina, F. Busqué, J. Hernando Langmuir 2008, 24, 2963.

[531] A. Martínez-Otero, F. Busqué, J. Hernando, D. Ruiz-Molina, Nanoscale, 2010, 2, 1781.

[532]C. K. Jørgensen, Coord. Chem. Rev. 1966, 1, 164.

[533] S. Messaoudi, V. Robert, N. Guihéry, D. Maynau, Inorg. Chem. 2006, 45, 3212 and references cited therein.

[534] W. Kaim, B. Schwederski, Pure Appl. Chem. 2004, 76, 351 and references cited therein.

[535] C. G. Pierpont, R. M. Buchanan, Coord. Chem. Rev. 1981, 38, 45.

[536] D. A. Shultz in Magnetism: Molecules to Materials, Vol. II (Eds.: J. S. Miller, M. Drillon), Wiley-VCH, Weinheim, 2001, 81-306.

[537] A. Vlcek, Comments Inorg. Chem. 1994, 16, 207.

[538] R. M. Buchanan, C. G. Pierpont, J. Am. Chem. Soc. 1980, 102, 4951. 
[539] a) E. Evangelio, D. Ruiz-Molina, Eur. J. Inorg. Chem., 2005, 15, 2957 and references cited therein;

b) D. N. Hendrickson, C. G. Pierpont, Top. Curr. Chem. 2004, 234, 63; c) O. Sato, J. Tao, Y. -Z. Zhang, Angew. Chem. Int. Ed. 2007, 46, 2152.

[540] P. Gütlich, A. Dei, Angew. Chem. Int. Ed. Engl. 1997, 36, 2734

[541] a) D. M. Adams, B. Li, J. D. Simon, D. N. Hendrickson, Angew. Chem. Int. Ed. Engl. 1995, 34, 1481;

b) D. M. Adams, D. N. Hendrickson, J. Am. Chem. Soc. 1996, 118, 11515.

[542] F. V. R. Neuwahl , R. Righini , A. Dei, Chem. Phys. Lett. 2002, 352, 408.

[543] a) J. Tao, H. Maruyama, O. Sato, J. Am. Chem. Soc. 2006, 128, 1790; b) A. Cui, K. Takahashi, A. Fujishima, O. Sato, J. Photochem. Photobiol. A: Chem. 2004, 167, 69; b) O. Sato, S. Hayami, Z. -Z. Gu, K. Takahashi, R. Nakajima, K. Seki, A. Fujishima, J. Photochem. Photobiol. A Chem. 2002, 149, 111; c) 0. Sato, S. Hayami, Y. Einaga, Z. -Z. Gu, Bull. Chem. Soc. Jpn, 2003, 76, 443; d) O. Sato, S. Hayami, Z. -Z. Gu, K. Takahashi, R. Nakajima, A. Fujishima, Chem. Phys. Lett. 2002, 355, 169; e) O. Sato, S. Hayami, Z. -Z. Gu, R. Saki, R. Nakajima, A. Fujishima, Chem. Lett. 2001, 874;

[544] a) A. Caneschi, A. Dei, F. F. De Biani, P. Gütlich, V. Ksenofontov, G. Levchenko, A. Hoefer, F. Renz, Chem. Eur. J. 2001, 7, 3926; b) C. Roux, D. M. Adams, J. P. Itié, A. Polian, D. N. Hendrickson, M. Verdaguer, Inorg. Chem. 1996, 35, 2846; c) O. -S. Jung, C. G. Pierpont, J. Am. Chem. Soc. 1994, 116, 2229.

[545] I. Ratera, D. Ruiz-Molina, F. Renz, J. Ensling, K. Wurst, C. Rovira, Ph. Gütlich, J. Veciana, J. Am. Chem. Soc. 2003, 125, 1462.

[546] a) L. A. deLaire, R. C. Haltiwanger, C. G. Pierpont, Inorg. Chem. 1989, 28, 644; b) A. Y. Girgis, A. L. Balch, Inorg. Chem. 1975, 14, 2724; c) O. Hayaishi, M. Nozaki, Science 1969, 164, 389.

[547] T. K. Paine, T. Weyhermüller, L. D. Slep, F. Neewe, E. Bill, E. Bothe, K. Wieghardt, P. Chaudhuri, Inorg. Chem. 2004, 43, 7324.

[548] A. Y. Girgis, A. L. Balch, Inorg. Chem. 1975, 14, 2724.

[549] a) A. Caneschi, A. Cornia, A. Dei, Inorg. Chem. 1998, 37, 3419.

[550] S. K. Larsen, C. G. Pierpont, J. Am. Chem. Soc. 1988, 110, 1827

[551] O. Cador, F. Chabre, A. Dei, C. Sangregorio, J. V. Slageren, M. G. F. Vaz, Inorg. Chem. 2003, 42, 6432. 
[552] E. Evangelio, M. -L. Bonnet, M. Cabañas, M. Nakano, J. -P. sutter, A. Dei, V. Robert and D. RuizMolina, Chem. Eur. J. 2010, 16, 6666.

[553] D. Ruiz-Molina, K. Wurst, D. Hendrickson, C. Rovira, J. Veciana, Adv. Funct. Mater. 2002, $12,347$.

[554] a) D. Ruiz-Molina, L. N. Zakharov, A.L. Rheingold, D.N. Hendrickson, J. Phys. Chem. Sol 2004, 65, 831; b) D. Ruiz-Molina, J. Yoo, I. Guzei, A. L. Rheingold, D. N. Hendrickson, Chem. Commun. 1998, 2089.

[555] D. Ruiz-Molina, J. Veciana,K. Wurst, D. N. Hendrickson, C. Rovira, Inorg. Chem. 2000, 39, 617.

[556] E. Evangelio, Cl. Rodriguez-Blanco, Y. Coppel, D. N. Hendrickson, J. P. Sutter, J. Campo, D. RuizMolina, Solid State Sciences 2009, 11, 793.

[557] E. Evangelio, D. Ruiz-Molina, C. R. Chimie 2008, 11, 1137.

[558] I. Imaz, D. Maspoch, Cl. Rodríguez-Blanco, J. Manuel Pérez-Falcón, J. Campo, D. Ruiz-Molina Angew. Chem. Int. Ed. 2008, 47, 1857.

[559] J. W. Whittaker, Chem. Rev. 2003, 103, 2347.

[560] M. M. Whittaker, P. J. Kersten, D. Cullen, J. W. Whittaker, J. Biol. Chem. 1999, 274, 36226.

[561] M. S. Dooley, M. A. McGuirl, D. E. Brown, P. N. Turowski, W. S. Mclntire, P. F. Knowles, Nature 1991, 349, 262.

[562] G. Speier, Z. Tyeklar, P. Toth, E. Speier, S. Tisza, A. Rochenbauer, A. M. Whalen, N. Alkire, C. G. Pierpont, Inorg. Chem. 2001, 40, 5653.

[563] a) J. Rall, M. Wanner, M. Albrecht, F. M. Hornung, W. Kaim, Chem. Eur. J. 1999, 5, 2802. b) W. Kaim, M. Wanner, A. Knödler, S. Zalis, Inorg. Chim. Acta 2002, 337, 163.

[564] a) J. Rall, W. Kaim, J. Chem. Soc. Faraday Trans. 1994, 90, 2905. b) G. Speier, S. Tisza, Z. Tyeklar, C. W. Lange, C. G. Pierpont Inorg. Chem. 1994, 33, 2041.

[565] R. M. Buchanan, C. Wilson-Blumenberg, C. Trapp, S. K. Larsen, D. L. Green, C. G. Pierpont, Inorg. Chem. 1986, 25, 3070.

[566] W. Kaim, M. Wanner, A. Knödler, S. Zalis, Inorg. Chim. Acta 2002, 337, 163.

[567] S. Ye, B. Sarkar, M. Niemeyer, W. Kaim, Eur. J. Inorg. Chem. 2005, 4735.

[568] D. G. Lonnon, S. T. Lee, S. B. Colbran, J. Am. Chem. Soc. 2007, 129, 5800.

[569] B. A. Borgias, S. R. Cooper, Y. B. Koh, K. N. Raymond, Inorg. Chem. 1984, 23, 1009.

[570] B. O’Regan, M. Grätzel, Nature, 1991, 353, 737.

[571] J. Moser, S. Punchihewa, P. P. Infelta, M. Grätzel, Langmuir 1991, 7, 3012. 
[572] R. Rodríguez, M. A. Blesa, A. E. Regazzoni, J. Colloid Interface Sci. 1996, 177, 122.

[573] S.-C. Lin, J. G. Wang, P. Jacobson, X.-Q. Gong, A. Selloni, U. Diebold, J. Am. Chem. Soc. 2009, 131, 980.

[574] P. C. Redfern, P. Zapol, L. A. Curtiss, T. Rajh, M. C. Thurnauer, J. Phys. Chem. B 2003, 107, 11419.

[575] Y. Wang, K. Hang, N. A. Anderson T. Lian, J. Phys. Chem. B. 2003, 107, 9434.

[576] S. Verna, P. Kar, A. Das, D. K. Palit, J. N. Ghosh, J. Phys. Chem. C 2008, 112, 2918.

[577] W. R. Duncan, O. V. Prezhdo, Annu. Rev. Phys. Chem. 2007, 58, 143.

[578] Y. Liu, J. I. Dadap, D. Zimdars, K. B. Eisenthal, J. Phys. Chem. B 1999, 103, 2480.

[579] T. Rajh, L. X. Chen, K. Lukas, T. Liu, M. C. Thurnauer, D. M. Tiede, J. Phys. Chem. B 2002, 106, 10543.

[580] P. Persson, R. Bergström, S. Lunell, J. Phys. Chem. B 2000, 104, 10348.

[581] W. R. Duncan, O. V. Prezhdo, J. Phys. Chem. B 2005, 109, 365.

[582] R. Sánchez-de-Armas, J. Oviedo, M. A. S. Miguel, J. F. Sanz, J. Phys. Chem. C 2011, 115, 11293.

[583] E. L. Tae, S. J. Lee, J. K. Lee, S. S. Yoo, E. J. Kang, K. B. Yoon, J. Phys. Chem. B 2005, 109, 22513.

[584] R. Huber, J.-E. Moser, M. Grätzel, J. Wachtveitl, J. Phys. Chem. B 2002, 106, 6494.

[585] C. R. Rice, M. D. Ward, M. K. Nazeeruddin, M. Grätzel, New J. Chem. 2000, 24, 651.

[586] P. Kar, S. Verma, A. Das, H. N. Ghosh, J. Phys. Chem. B 2009, 113, 7970.

[587] S. Verma, P. Kar, A. Das, D. K. Palit, H. N. Ghosh, J. Phys. Chem. C 2008, 112, 2918.

[588] R. Marczak, F. Werner, J.-F. Gnichwitz, A. Hirsch, D. M. Guldi, W. Peukert, J. Phys. Chem. B 2009, $113,4669$.

[589] J.-F. Gnichwitz, R. Marczak, F. Werner, N. Lang, N. Jux, D. M. Guidi, W. Peukert, A. Hirsch, J. Am. Chem. Soc. 2010, 132, 17910.

[590] Y. Pellegrin, L. L. Pleux, E. Blart, A. Renaud, B. Chavillon, N. Szuwarski, M. Boujtita, L. Cario, S. Jobic, D. Jacquemin, F. Odobel, J. Phothochem. Photobiolog. Chem. 2011, 219, 235.

[591] S. Verma, P. Kar, A. Das, D. K. Palit, J. N. Ghosh, Chem. Eur. J. 2010, 16, 611.

[592] P. Kar, S. Verma, A. Sen, B. Ganguly, H. N. Ghosh, Inorg. Chem. 2010, 49, 4167.

[593] S. Verma, P. Kar, A. Das, D. K. Palit, J. N. Ghosh, Chem. Eur. J. 2011, 17, 1561.

[594] T. Banerjee, S. Rawalekar, A. Das, H. N. Ghosh, Eur. J. Inorg. Chem. 2011, 4187.

[595] I. Hod, M. Shalom, Z. Tachan, S. Rühle, A. Zaban, J. Phys. Chem. C 2010, 114, 10015.

[596] B.-K. An,, W. Hu, P. L. Burn, P. Meredith, J. Phys. Chem. C 2010, 114, 17964. 
[597] S. Manzhos, H. Segawa, K. Yamashita, Chem. Phys. Lett. 2011, 504, 230.

[598] G. Ramakrishna, S. Verma, D. A. Jose, D. K. Kumar, A. Das, D. K. Palit, H. N. Ghosh, J. Phys. Chem. B 2006, 110, 9012.

[599] S. Verma, A. Ghosh, A. Das, H. N. Ghosh, J. Phys. Chem. C 2010, 114, 8327.

[600] S. Verma, A. Ghosh, A. Das, H. N. Ghosh, Chem. Eur. J. 2011, 17, 3458.

[601] W. R. Duncan, C. F. Craig, O. V. Prezhdo, J. Am. Chem. Soc. 2007, 129, 8528.

[602] W. R. Duncan, W. M. Stier, O. V. Prezhdo, J. Am. Chem. Soc. 2005, 127, 7941.

[603] M. Vega-Arroyo, P. R. LeBreton, T. Rajh, P. Zapol, L. A. Curtiss, Chem. Phys. Lett. 2005, 406, 306.

[604] T. Rajh, O. Poluektov, A. A. Dubinski, G. Wiederrecht, M. C. Thurnauer, A. D. Trifunac, Chem. Phys. Lett. 2001, 344, 31.

[605] R. Mosurkal, J.-A. He, K. Yang, L. A. Samuelson J. Kumar, J. Photochem. Photobiolog. Chem. 2004, 168, 191.

[606] G. S. Tulevski, Q. Miao, M. Fukuto, R. Abram, B. Ocko, R. Pindak, M. L. Steigerwald, C. R. Kagan, C. Nuckolls, J. Am. Chem. Soc. 2004, 126, 15048.

[607] Z. Algharaibeh, P. G. Pickup, Electrochem. Commun. 2011, 13, 147.

[608]J. García-Cañadas, A. P. Meacham, L. M. Peter, M. D. Ward, Angew. Chem. Int. Ed. 2003, 42, 3011.

[609] C. S. Grange, A. J. H. M. Meijer, M. D. Ward, Dalton Trans. 2010, 39, 200.

[610]D. Natali, M. Caironi, M. Sampietro, A. P. Meacham, S. J. Vlckers, M. D. Ward, Opt. Mat. 2006, 28, 1362.

[611] L. X. Chen, T. Rajh, Z. Y. Wang, M. C. Thurnauer, J. Phys. Chem. B. 1997, 101, 10688.

[612] T. Rajh, J. M. Nedeljkovic, L. X. Chen, O. Poluektov, M. C. Thurnauer, J. Phys. Chem. B. 1999, 103, 3515.

[613] L. X. Chen, T. Liu, M. C. Thurnauer, R. Csencsits, T. Rajh, J. Phys. Chem. B 2002, 106, 8539.

[614] S. J. Hurst, H. C. Fry, D. J. Gosztola, T. Rajh, J. Phys. Chem. C 2011, 115, 620.

[615] A. Musumeci, D. Gosztola, T. Schiller, N. M. Dimitrijevic, V. Mujica, D. Martin, T. Rajh, J. Am. Chem. Soc. 2009, 131, 6040 .

[616] E. Kim, Y. Liu, C. J. Baker, R. Owens, S. Xiao, W. E. Bentley, G. F. Payne, Biomacromolecules 2011, 12,880 .

[617] E. Kim, Y. Liu, X.-W. Shi, X. Yang, S. E. Bentley, G. F. Payne, Adv. Funct. Mater. 2010, 20, 2683.

[618] Y. Zhang, Y. Thomas, E. Kim, G. F. Payne, J. Phys. Chem. B 2012, 116, 1579. 
[619] S. J. Clarke, C. A. Hollmann, Z. Zhang, D. Suffern, S. E. Bradforth, N. M. Dimitrijevic, W. G. Minarik, J. L. Nadeau, Nat. Mater. 2006, 5, 409.

[620] I. L. Medintz, M. H. Stewart, S. A. Trammell, K. Susumu, J. B. Delehanty, B. C. mei, J. S. Melinger, J. B. Blanco-Canosa, P. E. Dawson, H. Mattoussi, Nat. Mater. 2010, 9, 676.

[621] X. Ji, G. Pauli, T. Avellini H. B. Na, C. Yi, K. L. Knappenberger, H. Mattoussi, J. Am. Chem. Soc. 2012, $134,6006$.

[622] S. Banerjee, S. Kar, J. M. Perez, S. Santra, J. Phys. Chem. C 2009, 113, 9659. 